\title{
Basicity of Some Phosphines in THF
}

Andrew Streitwieser,* Arlene E. McKeown, Faraj Hasanayn and Nicole R. Davis

\section{Supporting Information}

Department of Chemistry, University of California, Berkeley, Berkeley CA 94720-1460

\section{Table of Contents}

Figure S1 Spectrum of a mixture of $\mathrm{Cy}_{3} \mathrm{P}$ and BTPI $\quad$ p. S1

Table S1 Equilibrium measurements p. S2

$\begin{array}{lll}\text { Figure } \mathrm{S} 2 & {\left[\mathrm{Et}_{3} \mathrm{P}\right] \text { and }[\mathrm{BPFI}]} & \text { p. S4 }\end{array}$

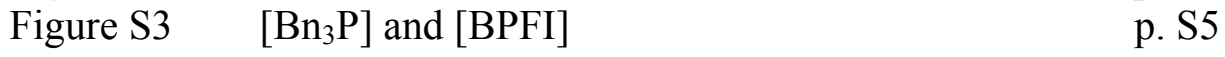

Table S2 HF 6-31Gd computations p. S5

Fig S4 pKa compared to PCM $\Delta \mathrm{E}+\mathrm{ZPE} \quad$ p. S52

Table S3. Computed PA of some phosphines p. S52

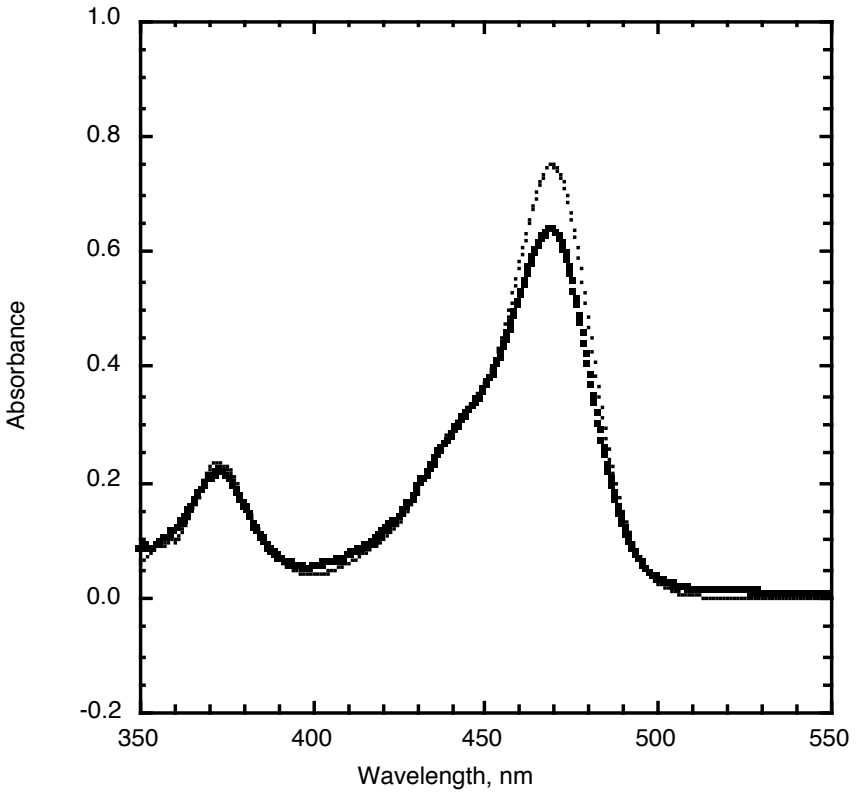

Figure S1. Spectrum of a mixture of $\mathrm{Cy}_{3} \mathrm{P}$ and BTPI (run 151) compared to the lithium salt of BTPI in THF. 
Table S1 Equilibrium measurements

\begin{tabular}{|c|c|c|c|c|c|}
\hline Run & {$[\text { Indicator }]_{0}^{\mathrm{a}}$} & {$\left[\mathrm{R}_{3} \mathrm{P}\right]_{0}$} & $\lambda_{\max } n \mathrm{~m}$ & $\left\{\mathrm{~A}^{-}\right\}^{\mathrm{b}}$ & Product $^{\mathrm{c}}$ \\
\hline \multicolumn{6}{|l|}{$\mathrm{Et}_{3} \mathrm{P}$} \\
\hline $\mathrm{s} 5 \mathrm{e} 1$ & $1.20 \mathrm{E}-2$ & $4.00 \mathrm{E}-2$ & 465.5 & $2.85 \mathrm{E}-05$ & $4.79 \mathrm{E}-04$ \\
\hline $\mathrm{s} 5 \mathrm{e} 2$ & $1.10 \mathrm{E}-2$ & $3.66 \mathrm{E}-2$ & 466.5 & $2.58 \mathrm{E}-05$ & $4.01 \mathrm{E}-04$ \\
\hline $\mathrm{s} 5 \mathrm{e} 3$ & $9.3 \mathrm{E}-3$ & $3.09 \mathrm{E}-2$ & 467.0 & $2.09 \mathrm{E}-05$ & $2.85 \mathrm{E}-04$ \\
\hline s5e4 & $8.1 \mathrm{E}-3$ & $2.69 \mathrm{E}-2$ & 467.5 & $1.77 \mathrm{E}-05$ & $2.16 \mathrm{E}-04$ \\
\hline s5e5 & 7.4E-3 & $2.47 \mathrm{E}-2$ & 468.0 & $1.61 \mathrm{E}-05$ & $1.83 \mathrm{E}-04$ \\
\hline s5e6 & $6.6 \mathrm{E}-3$ & $2.21 \mathrm{E}-2$ & 468.0 & $1.42 \mathrm{E}-05$ & $1.45 \mathrm{E}-04$ \\
\hline $\mathrm{s} 5 \mathrm{e} 7$ & $4.9 \mathrm{E}-3$ & $1.64 \mathrm{E}-2$ & 468.5 & $1.02 \mathrm{E}-05$ & $8.01 \mathrm{E}-05$ \\
\hline s5e8 & $3.7 \mathrm{E}-3$ & $1.24 \mathrm{E}-2$ & 469.0 & $7.49 \mathrm{E}-06$ & $4.56 \mathrm{E}-05$ \\
\hline s6e1 & $4.5 \mathrm{E}-3$ & $2.03 \mathrm{E}-2$ & 468.0 & $1.11 \mathrm{E}-05$ & $9.05 \mathrm{E}-05$ \\
\hline s6e 2 & $3.7 \mathrm{E}-3$ & $1.69 \mathrm{E}-2$ & 468.5 & $9.14 \mathrm{E}-06$ & $6.29 \mathrm{E}-05$ \\
\hline s6e3 & $2.7 \mathrm{E}-3$ & $1.22 \mathrm{E}-2$ & 469.0 & $6.46 \mathrm{E}-06$ & $3.29 \mathrm{E}-05$ \\
\hline s6e 4 & $1.7 \mathrm{E}-3$ & $7.8 \mathrm{E}-3$ & 469.5 & $4.23 \mathrm{E}-06$ & $1.32 \mathrm{E}-05$ \\
\hline \multicolumn{6}{|l|}{$\mathrm{Cy}_{3} \mathrm{P}$} \\
\hline $129 \mathrm{~b}$ & $2.15 \mathrm{E}-03$ & $1.35 \mathrm{E}-02$ & 468.0 & $2.79 \mathrm{E}-05$ & $2.86 \mathrm{E}-05$ \\
\hline $129 \mathrm{c}$ & $2.15 \mathrm{E}-03$ & $3.53 \mathrm{E}-02$ & 467.0 & $5.50 \mathrm{E}-05$ & $7.39 \mathrm{E}-05$ \\
\hline $129 \mathrm{~d}$ & $2.15 \mathrm{E}-03$ & $5.06 \mathrm{E}-02$ & 467.0 & $7.26 \mathrm{E}-05$ & $1.05 \mathrm{E}-04$ \\
\hline $129 \mathrm{e}$ & $2.15 \mathrm{E}-03$ & $6.31 \mathrm{E}-02$ & 466.5 & $8.75 \mathrm{E}-05$ & $1.30 \mathrm{E}-04$ \\
\hline $129 \mathrm{f}$ & $2.15 \mathrm{E}-03$ & $1.00 \mathrm{E}-01$ & 466.0 & $1.24 \mathrm{E}-04$ & $2.02 \mathrm{E}-04$ \\
\hline $129 \mathrm{~g}$ & $2.15 \mathrm{E}-03$ & $1.28 \mathrm{E}-01$ & 465.5 & $1.50 \mathrm{E}-04$ & $2.55 \mathrm{E}-04$ \\
\hline $129 \mathrm{~h}$ & $2.15 \mathrm{E}-03$ & $1.68 \mathrm{E}-01$ & 465.0 & $1.85 \mathrm{E}-04$ & $3.30 \mathrm{E}-04$ \\
\hline $130 \mathrm{~b}$ & $1.72 \mathrm{E}-03$ & $7.57 \mathrm{E}-03$ & 468.0 & $1.88 \mathrm{E}-05$ & $1.28 \mathrm{E}-05$ \\
\hline $130 \mathrm{c}$ & $1.72 \mathrm{E}-03$ & $1.80 \mathrm{E}-02$ & 467.0 & $3.17 \mathrm{E}-05$ & $3.04 \mathrm{E}-05$ \\
\hline $130 \mathrm{~d}$ & $1.72 \mathrm{E}-03$ & $3.48 \mathrm{E}-02$ & 467.0 & $5.11 \mathrm{E}-05$ & $5.80 \mathrm{E}-05$ \\
\hline $130 \mathrm{e}$ & $1.72 \mathrm{E}-03$ & $5.37 \mathrm{E}-02$ & 466.5 & $7.11 \mathrm{E}-05$ & $8.85 \mathrm{E}-05$ \\
\hline $130 \mathrm{f}$ & $1.72 \mathrm{E}-03$ & $7.77 \mathrm{E}-02$ & 467.0 & $9.35 \mathrm{E}-05$ & $1.26 \mathrm{E}-04$ \\
\hline $130 \mathrm{~g}$ & $1.72 \mathrm{E}-03$ & $1.06 \mathrm{E}-01$ & 466.0 & $1.21 \mathrm{E}-04$ & $1.70 \mathrm{E}-04$ \\
\hline $130 \mathrm{i}$ & $1.72 \mathrm{E}-03$ & $1.50 \mathrm{E}-01$ & 466.0 & $1.61 \mathrm{E}-04$ & $2.34 \mathrm{E}-04$ \\
\hline $146 \mathrm{~b}$ & $2.40 \mathrm{E}-03$ & $1.85 \mathrm{E}-04$ & 470.0 & $1.72 \mathrm{E}-06$ & $4.41 \mathrm{E}-07$ \\
\hline $146 c$ & $2.68 \mathrm{E}-03$ & $1.85 \mathrm{E}-04$ & 470.0 & $2.10 \mathrm{E}-06$ & $4.92 \mathrm{E}-07$ \\
\hline $146 \mathrm{~d}$ & $4.04 \mathrm{E}-03$ & $1.85 \mathrm{E}-04$ & 470.0 & $2.71 \mathrm{E}-06$ & $7.39 \mathrm{E}-07$ \\
\hline $146 \mathrm{e}$ & $6.04 \mathrm{E}-03$ & $1.85 \mathrm{E}-04$ & 469.5 & $3.49 \mathrm{E}-06$ & $1.10 \mathrm{E}-06$ \\
\hline $146 \mathrm{f}$ & $1.05 \mathrm{E}-02$ & $1.85 \mathrm{E}-04$ & 470.0 & $4.68 \mathrm{E}-06$ & $1.89 \mathrm{E}-06$ \\
\hline $146 \mathrm{~g}$ & $1.47 \mathrm{E}-02$ & $1.85 \mathrm{E}-04$ & 469.0 & $5.70 \mathrm{E}-06$ & $2.64 \mathrm{E}-06$ \\
\hline $146 \mathrm{~h}$ & $1.29 \mathrm{E}-02$ & $1.63 \mathrm{E}-04$ & 469.0 & $4.93 \mathrm{E}-06$ & $2.05 \mathrm{E}-06$ \\
\hline $146 \mathrm{i}$ & $1.15 \mathrm{E}-02$ & $1.45 \mathrm{E}-04$ & 469.5 & $4.24 \mathrm{E}-06$ & $1.63 \mathrm{E}-06$ \\
\hline $146 \mathrm{j}$ & $9.44 \mathrm{E}-03$ & 1.19E-04 & 469.5 & $3.28 \mathrm{E}-06$ & $1.09 \mathrm{E}-06$ \\
\hline $151 \mathrm{c}$ & $1.98 \mathrm{E}-03$ & $4.51 \mathrm{E}-04$ & 469.5 & $2.72 \mathrm{E}-06$ & $8.84 \mathrm{E}-07$ \\
\hline $151 \mathrm{~d}$ & $1.98 \mathrm{E}-03$ & $1.62 \mathrm{E}-03$ & 469.0 & $5.63 \mathrm{E}-06$ & $3.17 \mathrm{E}-06$ \\
\hline $151 \mathrm{e}$ & $1.98 \mathrm{E}-03$ & $2.59 \mathrm{E}-03$ & 469.0 & $7.50 \mathrm{E}-06$ & $5.08 \mathrm{E}-06$ \\
\hline $151 \mathrm{f}$ & $1.98 \mathrm{E}-03$ & $3.64 \mathrm{E}-03$ & 469.0 & $9.37 \mathrm{E}-06$ & $7.15 \mathrm{E}-06$ \\
\hline $151 \mathrm{~g}$ & $1.98 \mathrm{E}-03$ & $4.26 \mathrm{E}-03$ & 468.5 & $1.04 \mathrm{E}-05$ & $8.35 \mathrm{E}-06$ \\
\hline $151 \mathrm{~h}$ & $1.98 \mathrm{E}-03$ & $5.00 \mathrm{E}-03$ & 468.5 & $1.17 \mathrm{E}-05$ & $9.81 \mathrm{E}-06$ \\
\hline $151 \mathrm{i}$ & $1.98 \mathrm{E}-03$ & $6.38 \mathrm{E}-03$ & 468.5 & $1.37 \mathrm{E}-05$ & $1.25 \mathrm{E}-05$ \\
\hline $151 \mathrm{j}$ & $1.98 \mathrm{E}-03$ & $8.24 \mathrm{E}-03$ & 468.5 & $1.65 \mathrm{E}-05$ & $1.61 \mathrm{E}-05$ \\
\hline $151 \mathrm{k}$ & $1.76 \mathrm{E}-03$ & $7.34 \mathrm{E}-03$ & 468.5 & $1.43 \mathrm{E}-05$ & $1.28 \mathrm{E}-05$ \\
\hline 1511 & $1.57 \mathrm{E}-03$ & $6.55 \mathrm{E}-03$ & 468.5 & $1.24 \mathrm{E}-05$ & $1.02 \mathrm{E}-05$ \\
\hline $151 \mathrm{~m}$ & $1.33 \mathrm{E}-03$ & $5.54 \mathrm{E}-03$ & 468.5 & $1.02 \mathrm{E}-05$ & $7.30 \mathrm{E}-06$ \\
\hline $151 n$ & $1.12 \mathrm{E}-03$ & $4.68 \mathrm{E}-03$ & 469.0 & $8.18 \mathrm{E}-06$ & $5.20 \mathrm{E}-06$ \\
\hline 1510 & $9.58 \mathrm{E}-04$ & 3.99E-03 & 469.0 & $6.78 \mathrm{E}-06$ & $3.79 \mathrm{E}-06$ \\
\hline
\end{tabular}




\begin{tabular}{|c|c|c|c|c|c|}
\hline \multicolumn{6}{|l|}{$\mathrm{Bn}_{3} \mathrm{P}$} \\
\hline $135 b$ & $1.13 \mathrm{E}-03$ & $3.51 \mathrm{E}-02$ & 469.5 & $2.13 \mathrm{E}-05$ & $3.90 \mathrm{E}-05$ \\
\hline $135 \mathrm{c}$ & $1.13 \mathrm{E}-03$ & $7.53 \mathrm{E}-02$ & 468.5 & $4.01 \mathrm{E}-05$ & $8.23 \mathrm{E}-05$ \\
\hline $135 d$ & $1.13 \mathrm{E}-03$ & $1.25 \mathrm{E}-01$ & 467.5 & $5.84 \mathrm{E}-05$ & $1.34 \mathrm{E}-04$ \\
\hline $135 \mathrm{e}$ & $1.13 \mathrm{E}-03$ & $1.97 \mathrm{E}-01$ & 467.5 & $8.32 \mathrm{E}-05$ & $2.07 \mathrm{E}-04$ \\
\hline $135 \mathrm{f}$ & $1.13 \mathrm{E}-03$ & $2.64 \mathrm{E}-01$ & 466.5 & $1.01 \mathrm{E}-04$ & $2.72 \mathrm{E}-04$ \\
\hline $135 \mathrm{~g}$ & $1.13 \mathrm{E}-03$ & $3.45 \mathrm{E}-01$ & 466.5 & $1.24 \mathrm{E}-04$ & $3.49 \mathrm{E}-04$ \\
\hline $137 b$ & $2.03 \mathrm{E}-03$ & $1.70 \mathrm{E}-02$ & 469.0 & $2.00 \mathrm{E}-05$ & $2.00 \mathrm{E}-05$ \\
\hline $137 \mathrm{c}$ & $2.03 \mathrm{E}-03$ & $4.90 \mathrm{E}-02$ & 468.5 & $3.79 \mathrm{E}-05$ & $3.79 \mathrm{E}-05$ \\
\hline $137 d$ & $2.03 \mathrm{E}-03$ & $8.58 \mathrm{E}-02$ & 468.0 & $5.63 \mathrm{E}-05$ & $5.63 \mathrm{E}-05$ \\
\hline $137 \mathrm{e}$ & $2.03 \mathrm{E}-03$ & $1.48 \mathrm{E}-01$ & 467.0 & $8.12 \mathrm{E}-05$ & $8.12 \mathrm{E}-05$ \\
\hline $137 \mathrm{f}$ & $2.03 \mathrm{E}-03$ & $2.15 \mathrm{E}-01$ & 466.0 & $1.08 \mathrm{E}-04$ & $1.08 \mathrm{E}-04$ \\
\hline $137 \mathrm{~g}$ & $2.03 \mathrm{E}-03$ & $2.71 \mathrm{E}-01$ & 466.0 & $1.30 \mathrm{E}-04$ & $1.30 \mathrm{E}-04$ \\
\hline $137 \mathrm{~h}$ & $2.03 \mathrm{E}-03$ & $3.19 \mathrm{E}-01$ & 465.5 & $1.43 \mathrm{E}-04$ & $1.43 \mathrm{E}-04$ \\
\hline $137 \mathrm{i}$ & $2.03 \mathrm{E}-03$ & $3.94 \mathrm{E}-01$ & 465.0 & $1.76 \mathrm{E}-04$ & $1.76 \mathrm{E}-04$ \\
\hline $152 \mathrm{c}$ & $2.32 \mathrm{E}-03$ & $1.28 \mathrm{E}-03$ & 470.5 & $2.27 \mathrm{E}-06$ & $2.95 \mathrm{E}-06$ \\
\hline $152 d$ & $2.32 \mathrm{E}-03$ & $3.05 \mathrm{E}-03$ & 470.0 & $3.50 \mathrm{E}-06$ & $7.05 \mathrm{E}-06$ \\
\hline $152 \mathrm{e}$ & $2.32 \mathrm{E}-03$ & $3.21 \mathrm{E}-03$ & 469.5 & $4.76 \mathrm{E}-06$ & $7.41 \mathrm{E}-06$ \\
\hline $152 \mathrm{f}$ & $2.32 \mathrm{E}-03$ & $5.52 \mathrm{E}-03$ & 469.5 & $6.33 \mathrm{E}-06$ & $1.27 \mathrm{E}-05$ \\
\hline $152 \mathrm{~g}$ & $2.32 \mathrm{E}-03$ & $1.03 \mathrm{E}-02$ & 469.5 & $9.17 \mathrm{E}-06$ & $2.37 \mathrm{E}-05$ \\
\hline $152 \mathrm{~h}$ & $2.32 \mathrm{E}-03$ & $1.15 \mathrm{E}-02$ & 469.5 & $9.93 \mathrm{E}-06$ & $2.64 \mathrm{E}-05$ \\
\hline $152 \mathrm{i}$ & $2.32 \mathrm{E}-03$ & $1.44 \mathrm{E}-02$ & 469.5 & $1.16 \mathrm{E}-05$ & $3.30 \mathrm{E}-05$ \\
\hline $152 \mathrm{j}$ & $2.32 \mathrm{E}-03$ & $1.87 \mathrm{E}-02$ & 469.5 & $1.41 \mathrm{E}-05$ & $4.29 \mathrm{E}-05$ \\
\hline $154 \mathrm{c}$ & $2.07 \mathrm{E}-03$ & $2.17 \mathrm{E}-03$ & 469.5 & $1.64 \mathrm{E}-06$ & $4.48 \mathrm{E}-06$ \\
\hline $154 d$ & $2.07 \mathrm{E}-03$ & $4.27 \mathrm{E}-03$ & 469.5 & $3.11 \mathrm{E}-06$ & $8.80 \mathrm{E}-06$ \\
\hline $154 \mathrm{e}$ & $2.07 \mathrm{E}-03$ & $8.13 \mathrm{E}-03$ & 469.5 & $5.80 \mathrm{E}-06$ & $1.67 \mathrm{E}-05$ \\
\hline $154 \mathrm{f}$ & $2.07 \mathrm{E}-03$ & $1.10 \mathrm{E}-02$ & 469.5 & $7.65 \mathrm{E}-06$ & $2.26 \mathrm{E}-05$ \\
\hline $154 \mathrm{~g}$ & $2.07 \mathrm{E}-03$ & $1.50 \mathrm{E}-02$ & 469.0 & $1.03 \mathrm{E}-05$ & $3.09 \mathrm{E}-05$ \\
\hline $154 \mathrm{~h}$ & $2.07 \mathrm{E}-03$ & $1.86 \mathrm{E}-02$ & 469.0 & $1.25 \mathrm{E}-05$ & $3.82 \mathrm{E}-05$ \\
\hline $154 \mathrm{i}$ & $2.07 \mathrm{E}-03$ & $2.00 \mathrm{E}-02$ & 469.0 & $1.35 \mathrm{E}-05$ & $4.11 \mathrm{E}-05$ \\
\hline $154 \mathrm{j}$ & $2.07 \mathrm{E}-03$ & $2.29 \mathrm{E}-02$ & 469.0 & $1.53 \mathrm{E}-05$ & $4.70 \mathrm{E}-05$ \\
\hline $154 \mathrm{k}$ & $2.07 \mathrm{E}-03$ & $2.40 \mathrm{E}-02$ & 469.0 & $1.60 \mathrm{E}-05$ & $4.92 \mathrm{E}-05$ \\
\hline
\end{tabular}

(a). Initial concentration of 1,3-Bis-(pentafluorophenyl)indene (BPFI)

(b). absorbance $/ 7123.1$ (for $0.1 \mathrm{~mm}$ cell, at $\mathrm{l}_{\max }$ ); Formal concentration of indicator anion.

(c). $\left([\text { Indicator }]_{0}-\left\{\mathrm{A}^{-}\right\}\right)\left(\left[\mathrm{R}_{3} \mathrm{P}\right]_{0}-\left\{\mathrm{A}^{-}\right\}\right)$ 


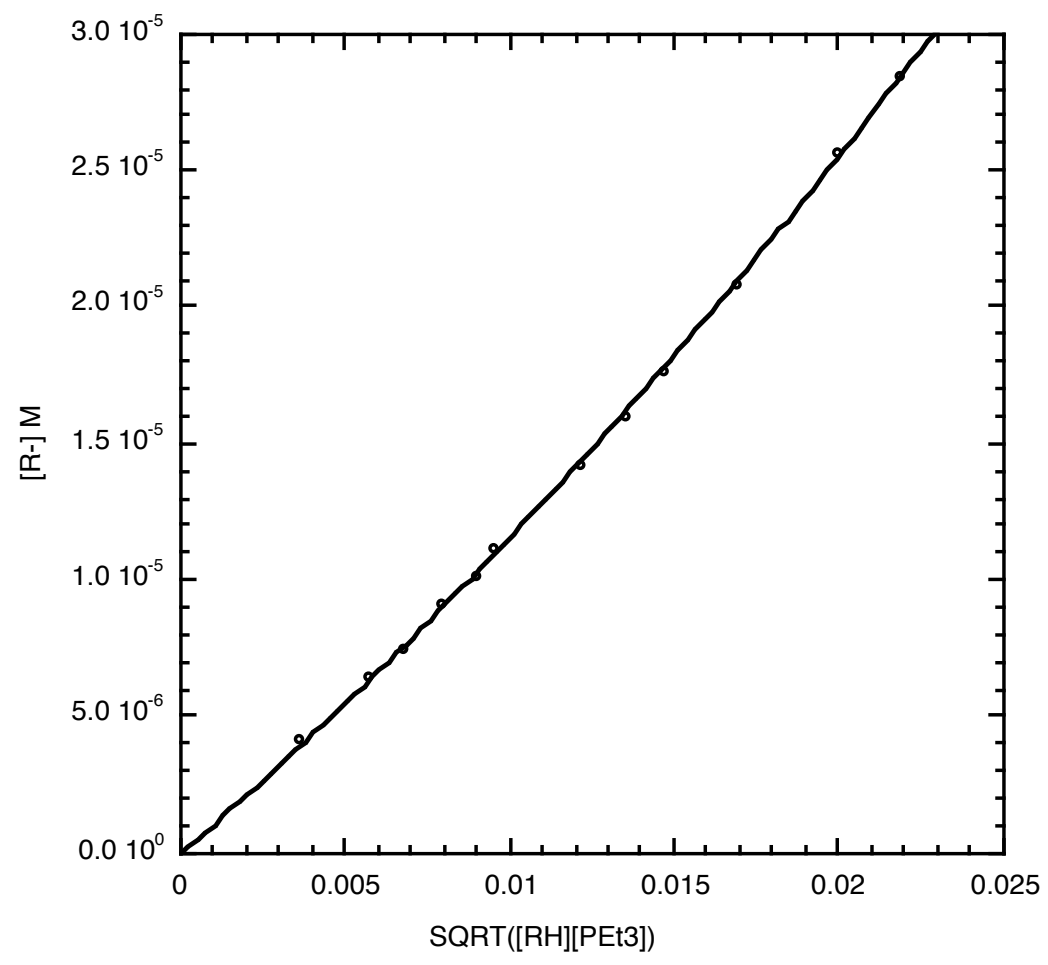

Figure S2. Plot of the formal indicator anion concentration $\left\{\mathrm{A}^{-}\right\}$vs the square root of the product of $\left[\mathrm{Et}_{3} \mathrm{P}\right]$ and $[\mathrm{BPFI}]$ at equilibrium. The second order curve shown through the origin is $\mathrm{y}=(1.032 \pm 0.014) \mathrm{E}-3 \mathrm{x}+(0.01219 \pm 0.00080) \mathrm{x}^{2} ; \mathrm{R}^{2}=0.9995$. 


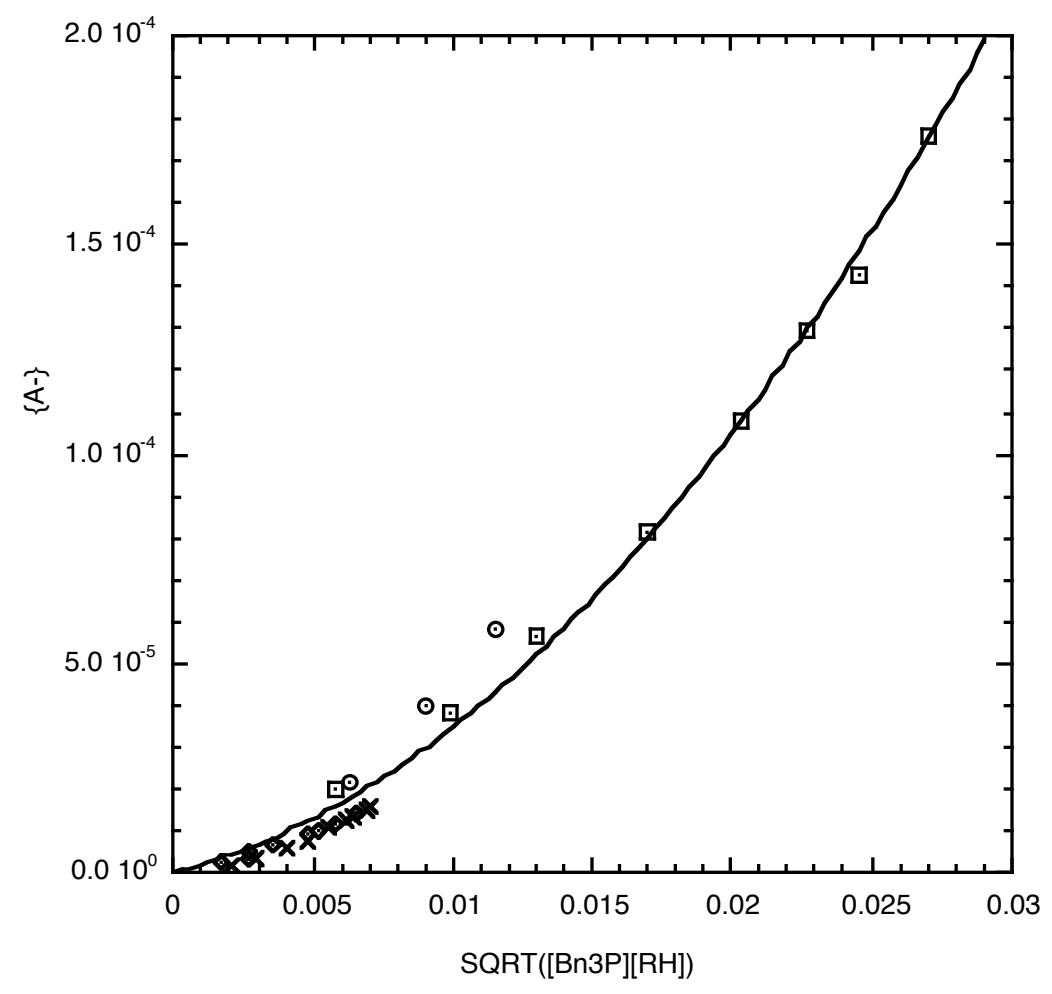

Figure S3. Plot of the formal indicator anion concentration $\left\{\mathrm{A}^{-}\right\}$vs the square root of the product of $\left[\mathrm{Bn}_{3} \mathrm{P}\right]$ and $[\mathrm{BPFI}]$ at equilibrium. The second order curve shown through the origin is $\mathrm{y}=(2.229 \pm 0.040) \mathrm{E}-3 \mathrm{x}+(0.1650 \pm 0.0201) \mathrm{x}^{2} ; \mathrm{R}^{2}=0.969$.

Table S2 HF 6-31Gd computations. PCM (polarized continuum model) results are listed as calculated by Gaussian03 for nitromethane as the solvent.

\section{Methylphosphine}

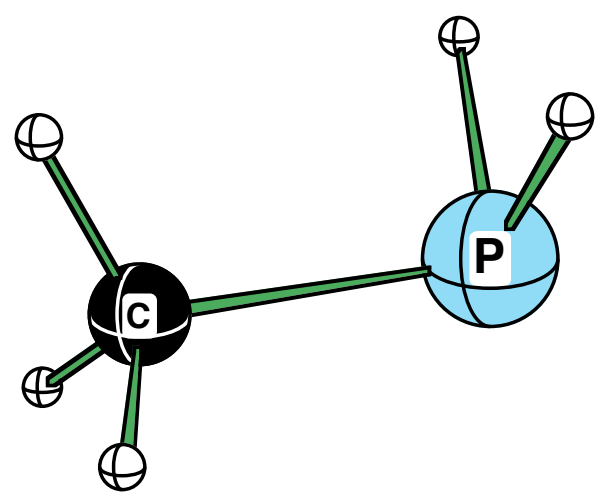

Coordinates (All structures optimized at HF 6-31+G*)

$\begin{array}{cccc}15 & .222438 & -.617587 & .157285 \\ 1 & .365380 & -.344316 & 1.527128 \\ 1 & 1.561587 & -.344307 & -.164549\end{array}$




$$
\begin{array}{cccc}
6 & -.528127 & .999611 & -.373440 \\
1 & -.544692 & 1.045737 & -1.456675 \\
1 & -1.554936 & 1.045727 & -.027989 \\
1 & .004858 & 1.863295 & .003447 \\
\mathrm{E}(\mathrm{RHF})=-381.486256750 & \text { atomic units } \\
\mathrm{ZPE}=36.79848 \text { (Kcal/Mol) }
\end{array}
$$

\section{Methylphosphonium Cation}

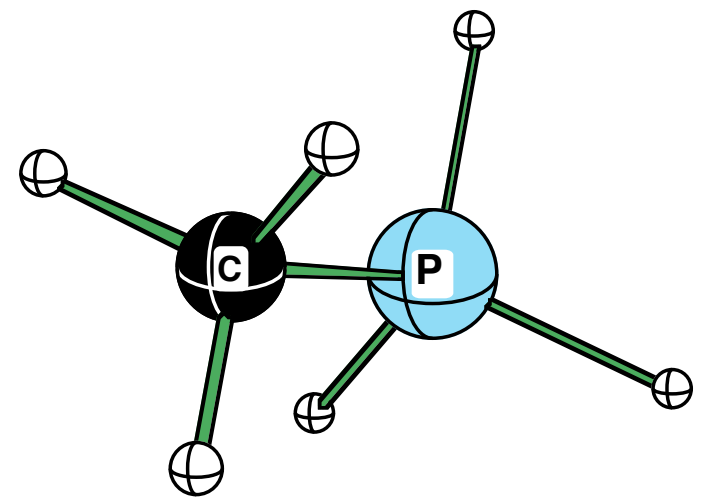

$$
\text { Coordinates }
$$

$\begin{array}{cccc}15 & -.020592 & .035674 & -.014566 \\ 1 & .005792 & -.010046 & 1.364506 \\ 1 & 1.288409 & -.010031 & -.449369 \\ 6 & -.874485 & 1.514658 & -.618354 \\ 1 & -.874531 & 1.514758 & -1.701387 \\ 1 & -1.895599 & 1.514744 & -.257399 \\ 1 & -.364015 & 2.399012 & -.257383 \\ 1 & -.635500 & -1.120807 & -.449386 \\ \mathrm{E}(\mathrm{RHF})=-381.825377723 \\ \mathrm{ZPE}=44.34800\end{array}$

\section{Dimethylphosphine}

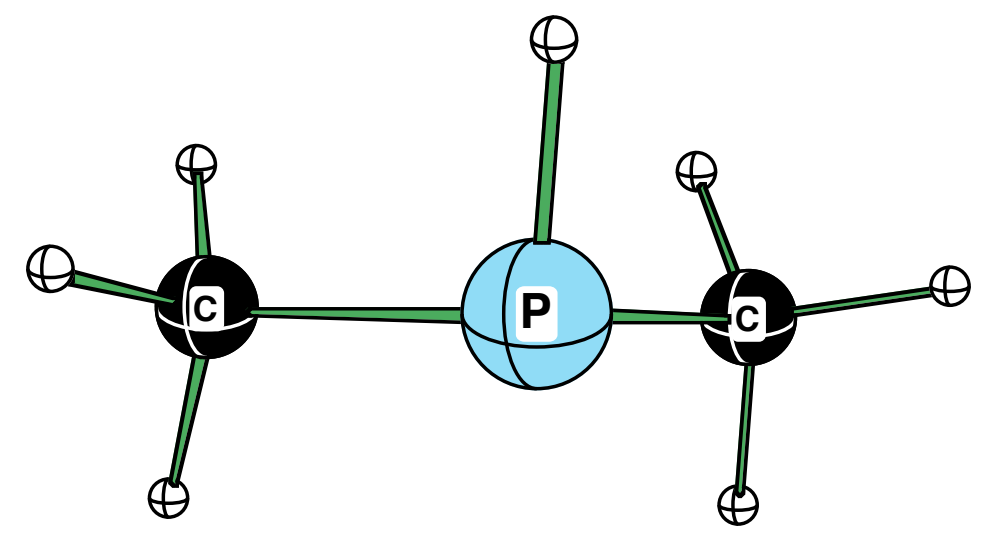

Coordinates

$\begin{array}{llll}15 & -.101983 & .176633 & -.094535\end{array}$ 


$\begin{array}{lccc}1 & .052287 & -.090535 & 1.276658 \\ 6 & 1.671481 & -.035159 & -.599378 \\ 1 & 1.744650 & .001150 & -1.681449 \\ 1 & 2.262334 & .784176 & -.204112 \\ 1 & 2.100832 & -.970148 & -.256210 \\ 6 & -.805283 & -1.465144 & -.599345 \\ 1 & -1.810269 & -1.567176 & -.204066 \\ 1 & -.873325 & -1.510374 & -1.681414 \\ 1 & -.210221 & -2.304455 & -.256170 \\ \text { E(RHF })=-420.526107661 \\ \text { ZPE }=56.50792\end{array}$

Dimethylphosphonium Cation

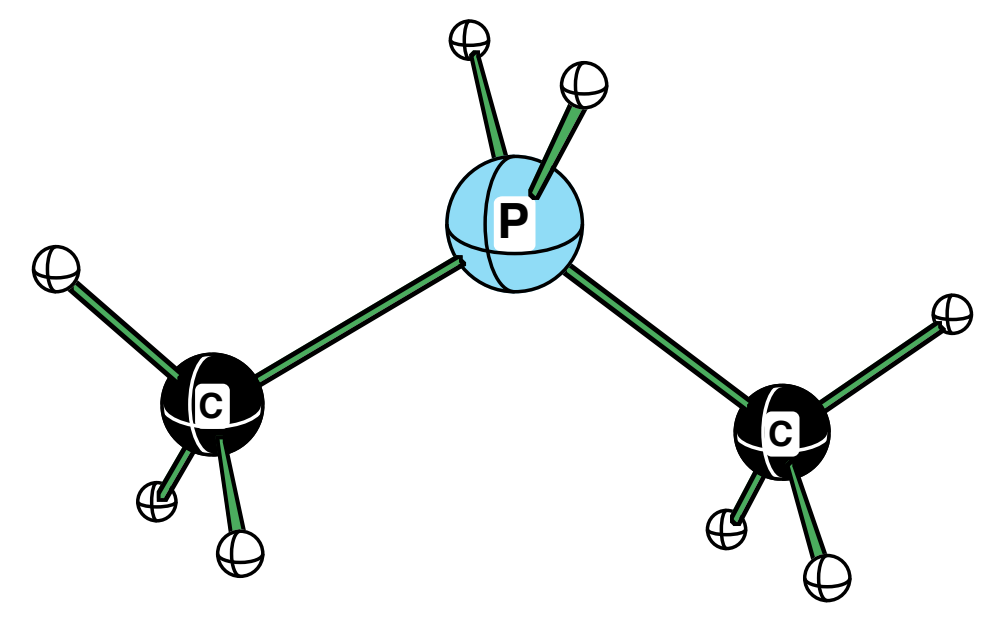

Coordinates

$$
\begin{array}{cccc}
15 & .028087 & -.048649 & -.039746 \\
1 & .004930 & -.008522 & 1.340386 \\
6 & 1.743618 & .003837 & -.614120 \\
1 & 1.766644 & .014450 & -1.697202 \\
1 & 2.229077 & .896599 & -.239121 \\
1 & 2.276460 & -.868487 & -.255177 \\
6 & -.875125 & -1.508111 & -.614100 \\
1 & -1.890994 & -1.482176 & -.239058 \\
1 & -.895874 & -1.522733 & -1.697182 \\
1 & -.386057 & -2.405721 & -.255188 \\
1 & -.630232 & 1.091575 & -.456179 \\
\mathrm{E}(\mathrm{RHF})=-420.886278902 \\
\mathrm{ZPE}=63.88685
\end{array}
$$

\section{Trimethylphosphine}




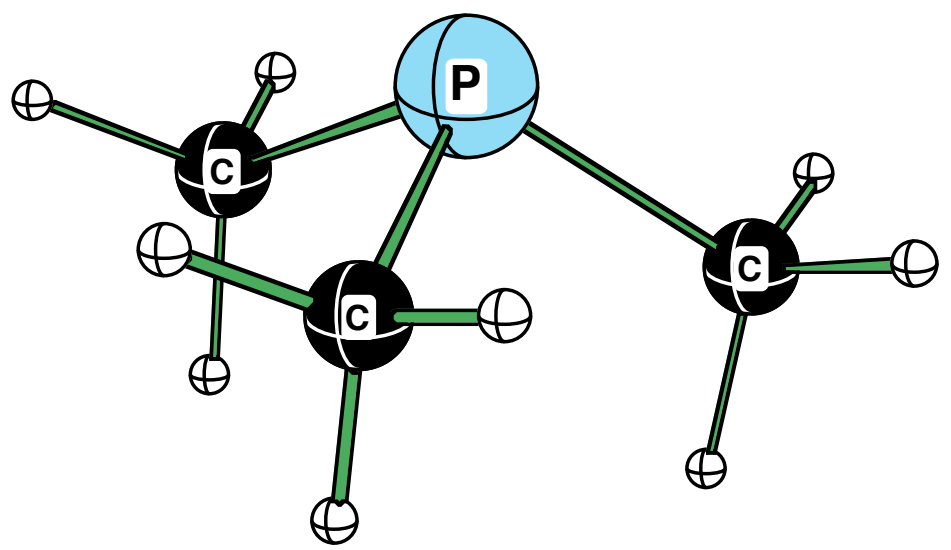

Coordinates

$\begin{array}{cccc}15 & -0.184143 & -0.505228 & -0.244182 \\ 6 & -0.246845 & -0.365192 & 1.602598 \\ 1 & 0.358451 & -1.146458 & 2.050944 \\ 1 & 0.110383 & 0.596150 & 1.961082 \\ 1 & -1.266729 & -0.503650 & 1.946751 \\ 6 & -1.066784 & 1.054432 & -0.715928 \\ 1 & -0.660663 & 1.931047 & -0.219025 \\ 1 & -0.999312 & 1.204479 & -1.788639 \\ 1 & -2.118879 & 0.971693 & -0.462883 \\ 6 & 1.569267 & 0.012046 & -0.547730 \\ 1 & 1.740772 & 0.120105 & -1.613881 \\ 1 & 2.246384 & -0.753943 & -0.183644 \\ 1 & 1.817908 & 0.951280 & -0.061610 \\ \mathrm{E}(\mathrm{RHF})=-459.567373839 & \\ \mathrm{ZPE}=75.79664 & \end{array}$

Total PCM free energy in MeNO2= -459.561281

Trimethylphosphonium Cation

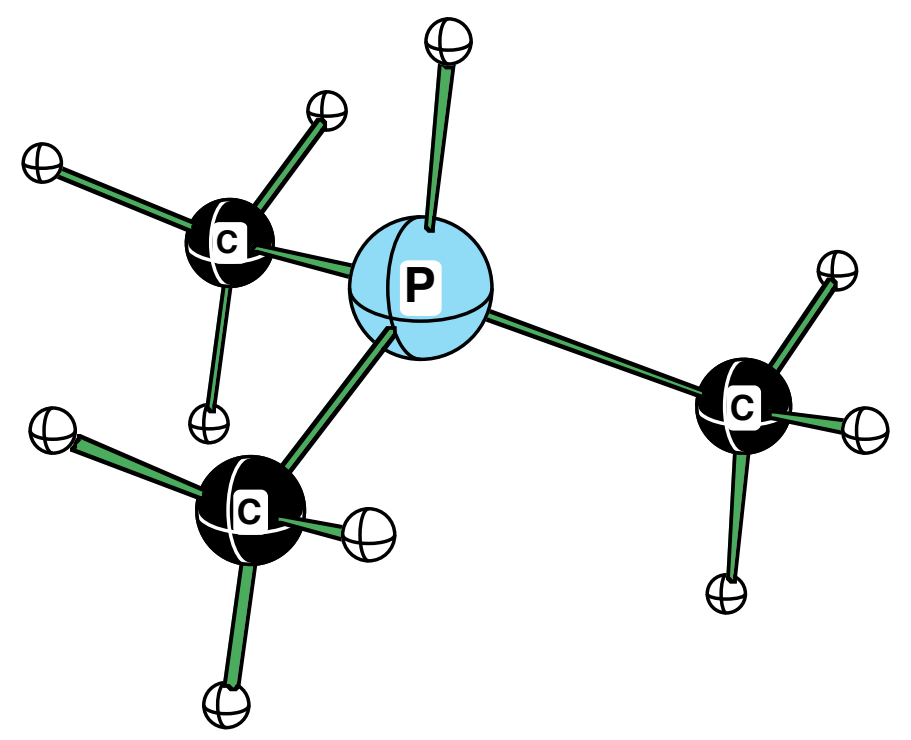




\section{Coordinates}

$\begin{array}{cccl}15 & .000002 & .000056 & -.062697 \\ 1 & .000009 & .000238 & 1.319393 \\ 6 & 1.723201 & .000052 & -.615071 \\ 1 & 1.762512 & -.000348 & -1.698209 \\ 1 & 2.231867 & .882081 & -.244825 \\ 1 & 2.232082 & -.881579 & -.244169 \\ 6 & -.861549 & -1.492384 & -.614861 \\ 1 & -.352188 & -2.373842 & -.244209 \\ 1 & -1.879606 & -1.492087 & -.244240 \\ 1 & -.881252 & -1.526552 & -1.697994 \\ 6 & -.861654 & 1.492289 & -.615254 \\ 1 & -1.879736 & 1.491979 & -.244702 \\ 1 & -.352402 & 2.373879 & -.244765 \\ 1 & -.881287 & 1.526214 & -1.698396 \\ \text { E(RHF) }=-459.944970629 \\ \text { ZPE= 83.11824 } \\ \text { PCM E (in MeNO2)=-460.017127 }\end{array}$

Triethylphosphine

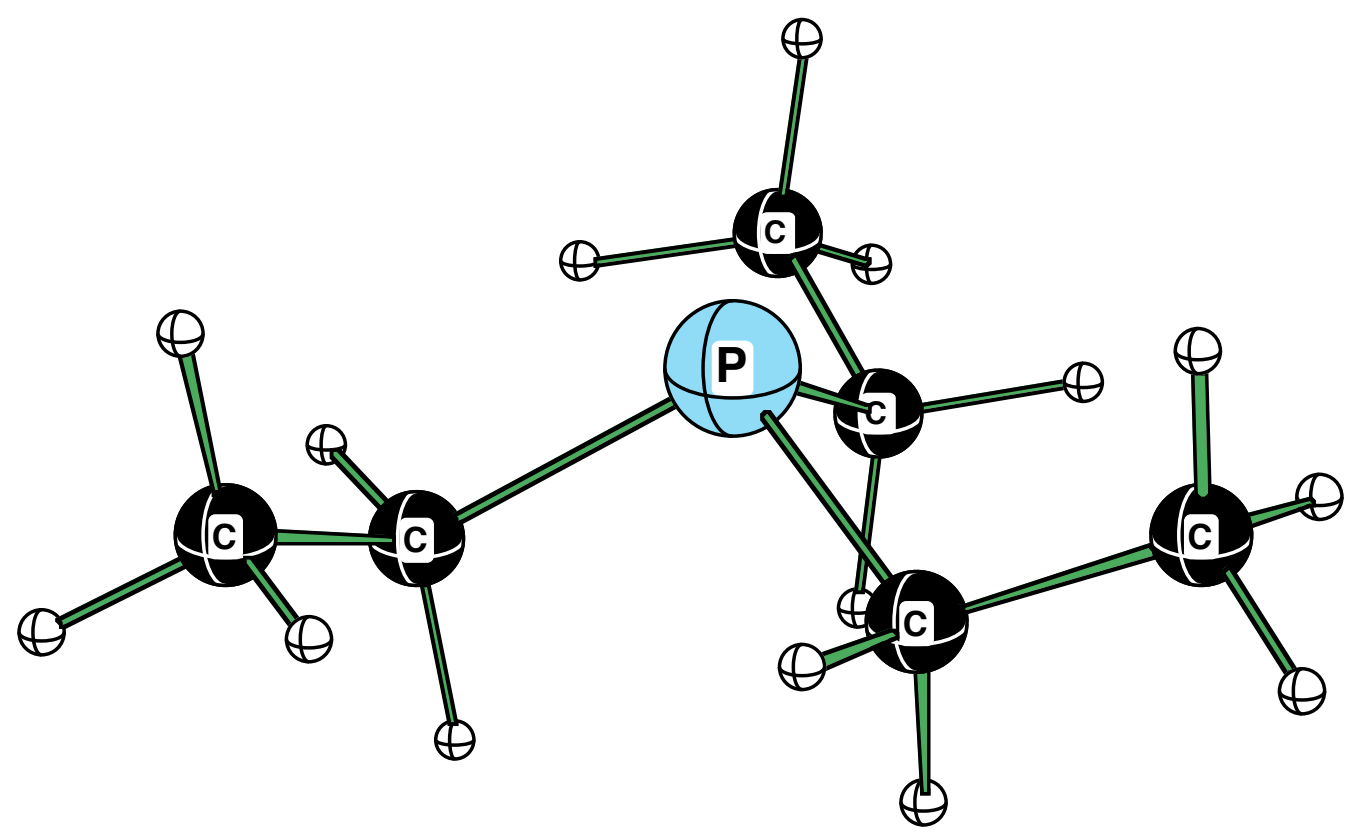

Coordinates

$\begin{array}{cccl}15 & -0.456972 & -0.069565 & -0.124096 \\ 6 & -0.074901 & 0.115179 & 1.689902 \\ 1 & 0.973658 & 0.370217 & 1.827734 \\ 1 & -0.220960 & -0.858462 & 2.148685 \\ 6 & 0.773505 & -1.388100 & -0.591195 \\ 1 & 0.890723 & -1.348847 & -1.670317 \\ 1 & 1.747476 & -1.159871 & -0.163634\end{array}$




$\begin{array}{cccc}6 & 0.399601 & 1.440236 & -0.800489 \\ 1 & -0.035961 & 2.303894 & -0.306357 \\ 1 & 1.453346 & 1.425521 & -0.530449 \\ 6 & 0.339517 & -2.801982 & -0.191323 \\ 1 & 0.244041 & -2.906343 & 0.884678 \\ 1 & -0.617457 & -3.057316 & -0.634185 \\ 1 & 1.068005 & -3.534847 & -0.524572 \\ 6 & -0.965190 & 1.137528 & 2.403694 \\ 1 & -0.732198 & 1.174418 & 3.463504 \\ 1 & -0.829184 & 2.138541 & 2.007151 \\ 1 & -2.014491 & 0.881394 & 2.301305 \\ 6 & 0.245152 & 1.606408 & -2.315692 \\ 1 & -0.801006 & 1.652889 & -2.599442 \\ 1 & 0.723292 & 2.521600 & -2.651321 \\ 1 & 0.699182 & 0.785084 & -2.860724 \\ \text { E(RHF) }=-576.663204868 \\ \text { ZPE=133.79705 } \\ \text { PCM E (in MeNO2)=-576.653785 }\end{array}$

\section{Triethylphosphonium Cation}

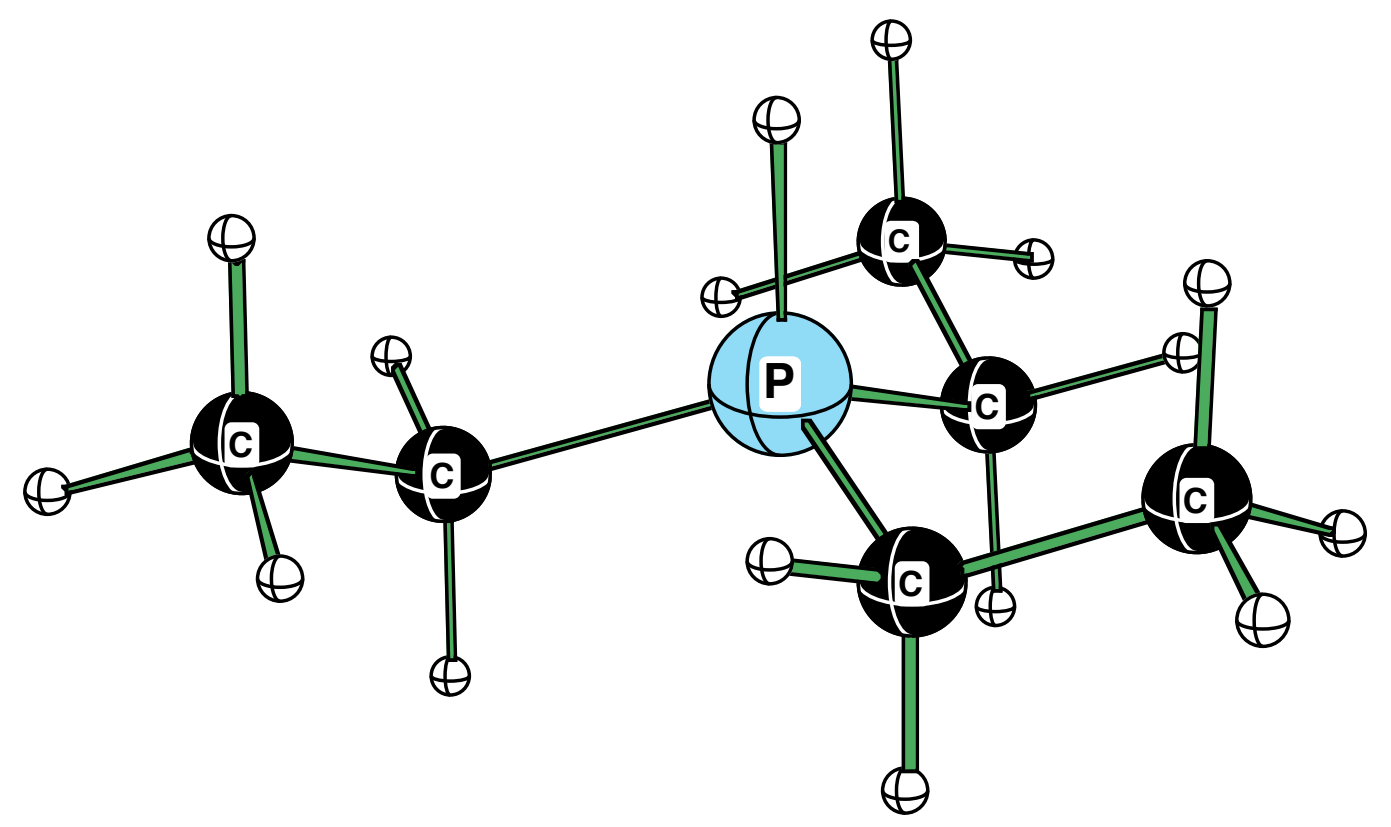

Coordinates

$$
\begin{array}{rrrr}
15 & -0.178948 & 0.018498 & -0.093901 \\
6 & -0.507426 & -0.009462 & 1.697934 \\
1 & 0.447260 & -0.141702 & 2.198041 \\
1 & -1.095235 & -0.898323 & 1.898610 \\
6 & 0.571964 & -1.541626 & -0.660907 \\
1 & 0.750600 & -1.438897 & -1.725520 \\
1 & 1.543687 & -1.623249 & -0.183297 \\
6 & 0.832455 & 1.458365 & -0.566341
\end{array}
$$




$\begin{array}{lrrr}1 & 0.330397 & 2.338692 & -0.180561 \\ 1 & 1.777151 & 1.375439 & -0.037483 \\ 6 & -0.282670 & -2.787535 & -0.381334 \\ 1 & -0.405520 & -2.967032 & 0.679434 \\ 1 & -1.263278 & -2.713404 & -0.837632 \\ 1 & 0.211275 & -3.652783 & -0.803934 \\ 6 & -1.232803 & 1.239293 & 2.222616 \\ 1 & -1.438062 & 1.107654 & 3.277019 \\ 1 & -0.631984 & 2.133808 & 2.115849 \\ 1 & -2.180783 & 1.394744 & 1.720157 \\ 6 & 1.061473 & 1.595172 & -2.079512 \\ 1 & 0.126123 & 1.661638 & -2.623569 \\ 1 & 1.616387 & 2.504856 & -2.268647 \\ 1 & 1.638674 & 0.771081 & -2.479826 \\ 1 & -1.400441 & 0.144765 & -0.734864 \\ \text { E(RHF)=-577.050447264 } \\ \text { ZPE=141.23865 } \\ \text { PCM E(in MeNO2)=-577.113713 }\end{array}$

Dimethylphenylphosphine 


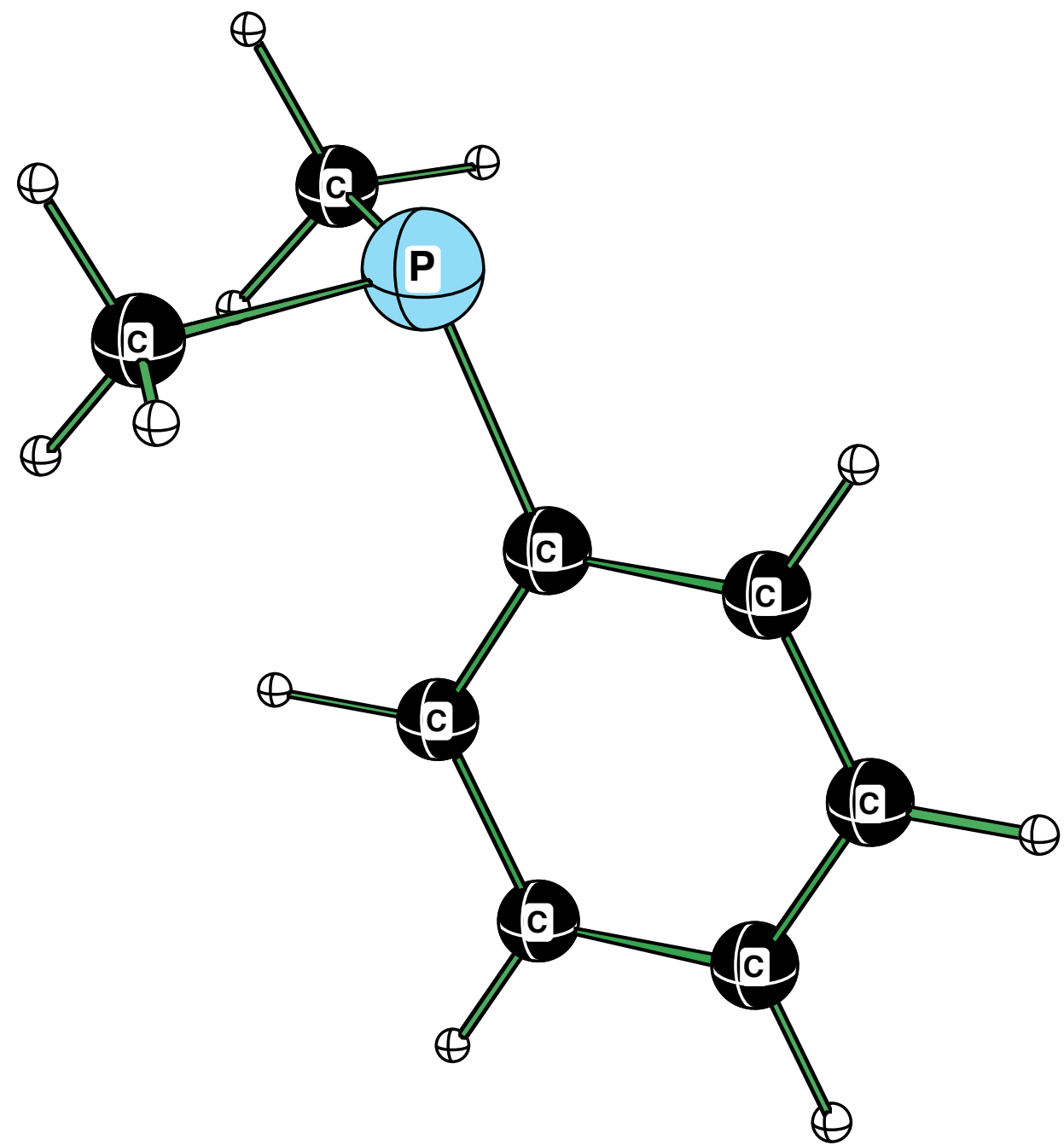

Coordinates

$\begin{array}{crrr}15 & -0.105738 & -0.136917 & -0.154182 \\ 6 & -0.014216 & 0.025416 & 1.690270 \\ 1 & 0.525506 & 0.907110 & 2.020219 \\ 1 & -1.021850 & 0.071515 & 2.091722 \\ 1 & 0.469260 & -0.850535 & 2.108462 \\ 6 & -0.716542 & 1.555900 & -0.598834 \\ 1 & -0.139376 & 2.356054 & -0.146956 \\ 1 & -0.691926 & 1.679649 & -1.675881 \\ 1 & -1.748609 & 1.655383 & -0.277035 \\ 6 & 1.675570 & -0.039604 & -0.635483 \\ 6 & 2.181867 & -1.039006 & -1.460044 \\ 6 & 3.510492 & -1.031786 & -1.862593 \\ 6 & 4.353463 & -0.019423 & -1.443029 \\ 6 & 3.865378 & 0.986135 & -0.619903 \\ 6 & 2.541071 & 0.974031 & -0.221997 \\ 1 & 1.532499 & -1.830011 & -1.790647 \\ 1 & 3.881025 & -1.814514 & -2.500402 \\ 1 & 5.383519 & -0.009911 & -1.752471\end{array}$




\author{
$\begin{array}{lllll}1 & 4.516974 & 1.776140 & -0.290593\end{array}$ \\ $\begin{array}{llll}1 & 2.183681 & 1.762749 & 0.415823\end{array}$ \\ $\mathrm{E}(\mathrm{RHF})=-650.075143965$ \\ $\mathrm{ZPE}=112.09520$ \\ $\mathrm{PCM}$ E(in MeNO2 $)=-650.072187$
}

\title{
Dimethylphenylphosphonium Cation
}

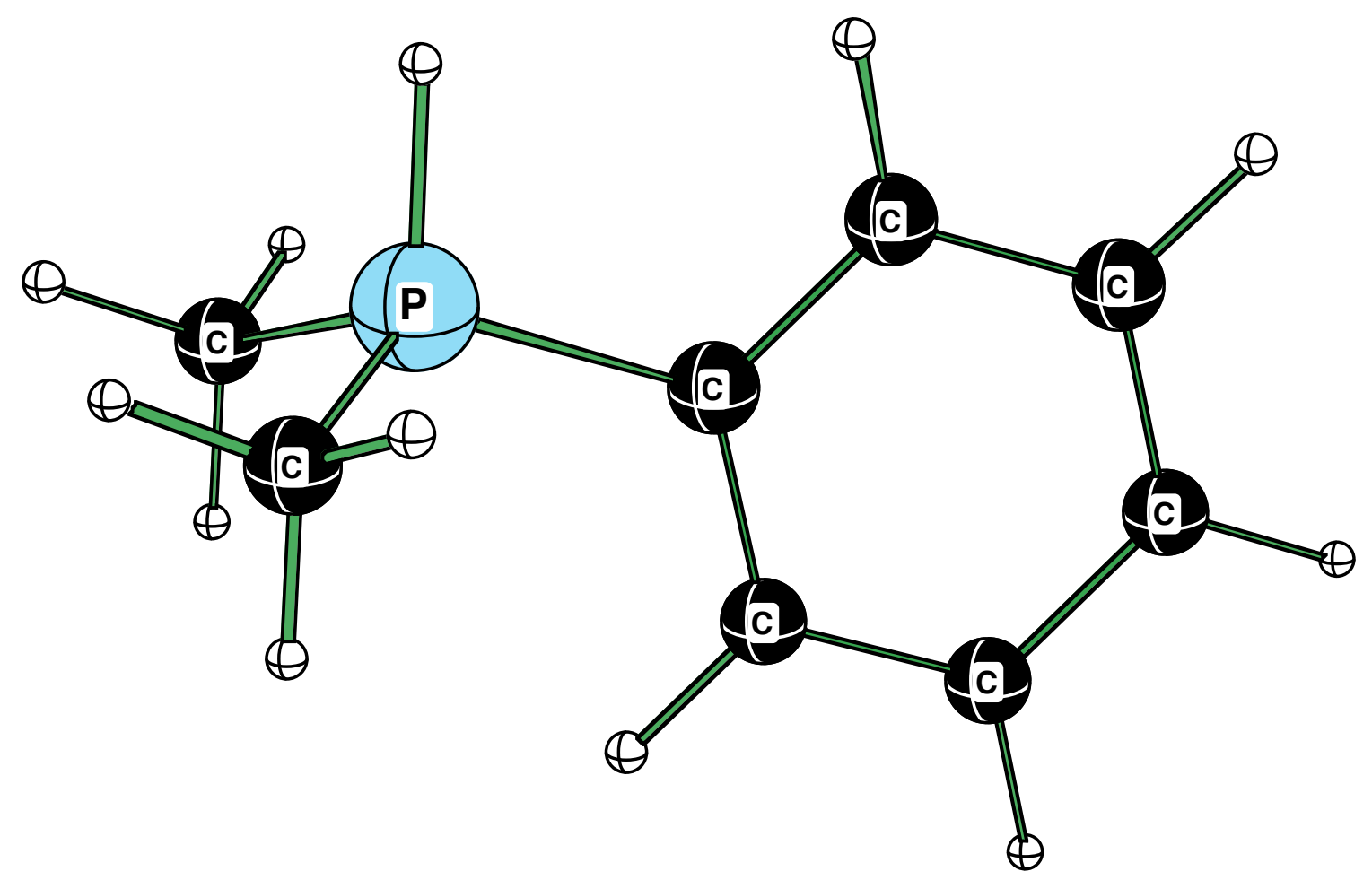

Coordinates

$\begin{array}{crcc}15 & 0.268270 & -0.021635 & 0.128886 \\ 6 & -0.099136 & 0.031659 & 1.902189 \\ 1 & 0.822325 & 0.068190 & 2.471126 \\ 1 & -0.688871 & 0.911185 & 2.132505 \\ 1 & -0.650579 & -0.855430 & 2.187782 \\ 6 & 1.114276 & 1.500857 & -0.369811 \\ 1 & 2.068871 & 1.578088 & 0.137035 \\ 1 & 1.286705 & 1.489516 & -1.438789 \\ 1 & 0.509603 & 2.362543 & -0.112380 \\ 6 & 1.162067 & -1.496533 & -0.347388 \\ 6 & 0.579275 & -2.394812 & -1.238771 \\ 6 & 1.268300 & -3.537266 & -1.609311 \\ 6 & 2.530038 & -3.780103 & -1.092991 \\ 6 & 3.114455 & -2.886331 & -0.203664 \\ 6 & 2.435112 & -1.745165 & 0.171203 \\ 1 & -0.399975 & -2.214642 & -1.644867\end{array}$




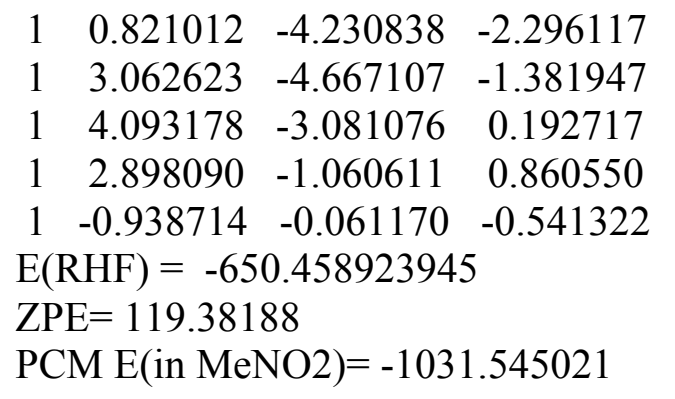

\section{Methyldiphenylphosphine}

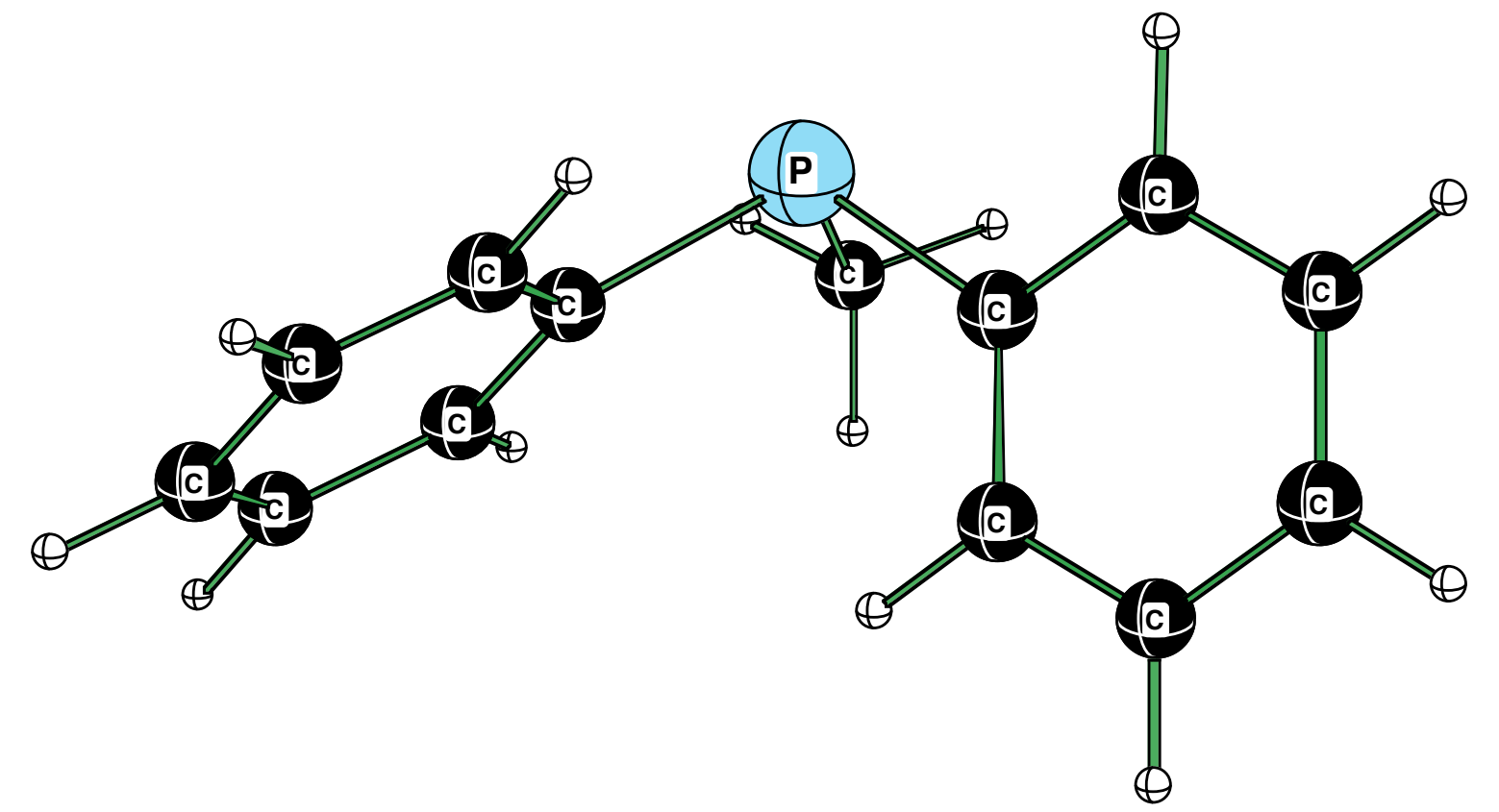

Coordinates

$\begin{array}{cccc}15 & 0.063746 & -0.225203 & -0.115786 \\ 6 & 0.122083 & 0.071784 & 1.711069 \\ 1 & 1.164271 & 0.082462 & 2.010450 \\ 1 & -0.363989 & -0.742021 & 2.238528 \\ 1 & -0.327365 & 1.010978 & 2.014124 \\ 6 & -1.720948 & 0.021229 & -0.522955 \\ 6 & -2.725868 & 0.184561 & 0.424160 \\ 6 & -4.055337 & 0.303547 & 0.039980 \\ 6 & -4.400546 & 0.265118 & -1.297655 \\ 6 & -3.409202 & 0.098560 & -2.254763 \\ 6 & -2.088649 & -0.030308 & -1.869438 \\ 1 & -2.488445 & 0.220146 & 1.470771 \\ 1 & -4.815899 & 0.428323 & 0.790425 \\ 1 & -5.429725 & 0.359330 & -1.595022 \\ 1 & -3.667391 & 0.062829 & -3.298247 \\ 1 & -1.332462 & -0.169645 & -2.622456\end{array}$




$\begin{array}{llll}6 & 0.827861 & 1.337596 & -0.739640 \\ 6 & 0.239780 & 2.595347 & -0.601021 \\ 6 & 0.879332 & 3.730511 & -1.063709 \\ 6 & 2.122041 & 3.629718 & -1.674684 \\ 6 & 2.716333 & 2.390178 & -1.822772 \\ 6 & 2.069103 & 1.252061 & -1.360818 \\ 1 & -0.726666 & 2.686311 & -0.138697 \\ 1 & 0.411311 & 4.692449 & -0.951207 \\ 1 & 2.618110 & 4.513382 & -2.035163 \\ 1 & 3.676522 & 2.304030 & -2.299615 \\ 1 & 2.533170 & 0.289982 & -1.488079 \\ \mathrm{E}(\mathrm{RHF})=-840.582183023 \\ \mathrm{ZPE}=148.31613\end{array}$

\section{Methyldiphenylphosphonium Cation}

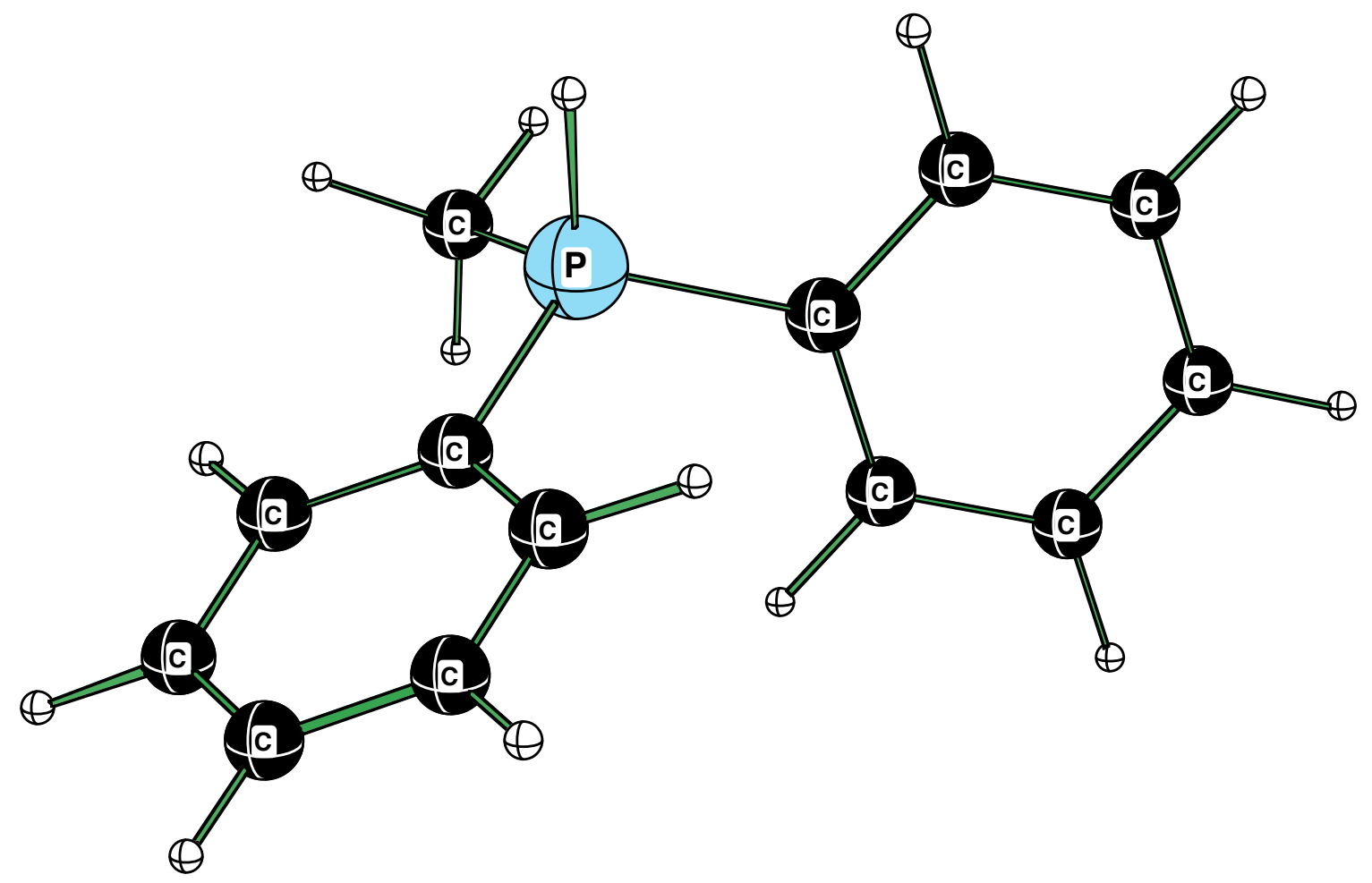

Coordinates

$\begin{array}{cccc}15 & -0.177149 & 0.327685 & 0.179375 \\ 6 & -0.039249 & 0.045604 & 1.964913 \\ 1 & 1.008813 & 0.072813 & 2.237076 \\ 1 & -0.446874 & -0.920344 & 2.235440 \\ 1 & -0.560150 & 0.823943 & 2.508737 \\ 6 & -1.889980 & 0.334339 & -0.357012 \\ 6 & -2.907986 & -0.106405 & 0.486823 \\ 6 & -4.215771 & -0.126043 & 0.034395\end{array}$




$\begin{array}{crrr}6 & -4.507473 & 0.287476 & -1.255230 \\ 6 & -3.496807 & 0.725756 & -2.100437 \\ 6 & -2.189857 & 0.752464 & -1.655839 \\ 1 & -2.698740 & -0.430415 & 1.489005 \\ 1 & -5.000358 & -0.462103 & 0.685938 \\ 1 & -5.523572 & 0.270541 & -1.603649 \\ 1 & -3.728176 & 1.046718 & -3.098698 \\ 1 & -1.414109 & 1.102451 & -2.312641 \\ 6 & 0.710064 & 1.802165 & -0.322007 \\ 6 & 0.157267 & 3.065076 & -0.101976 \\ 6 & 0.874442 & 4.193843 & -0.446512 \\ 6 & 2.137015 & 4.067836 & -1.011457 \\ 6 & 2.687142 & 2.816219 & -1.235519 \\ 6 & 1.975982 & 1.679564 & -0.891606 \\ 1 & -0.825083 & 3.169488 & 0.323008 \\ 1 & 0.452325 & 5.167095 & -0.279883 \\ 1 & 2.689826 & 4.948828 & -1.280522 \\ 1 & 3.661135 & 2.723666 & -1.678008 \\ 1 & 2.408408 & 0.711620 & -1.073389 \\ 1 & 0.491474 & -0.724630 & -0.419739 \\ \mathrm{E}(\mathrm{RHF})=-840.971929586 & \\ \mathrm{ZPE}=155.74798 & & \end{array}$

\section{Triphenylphosphine}

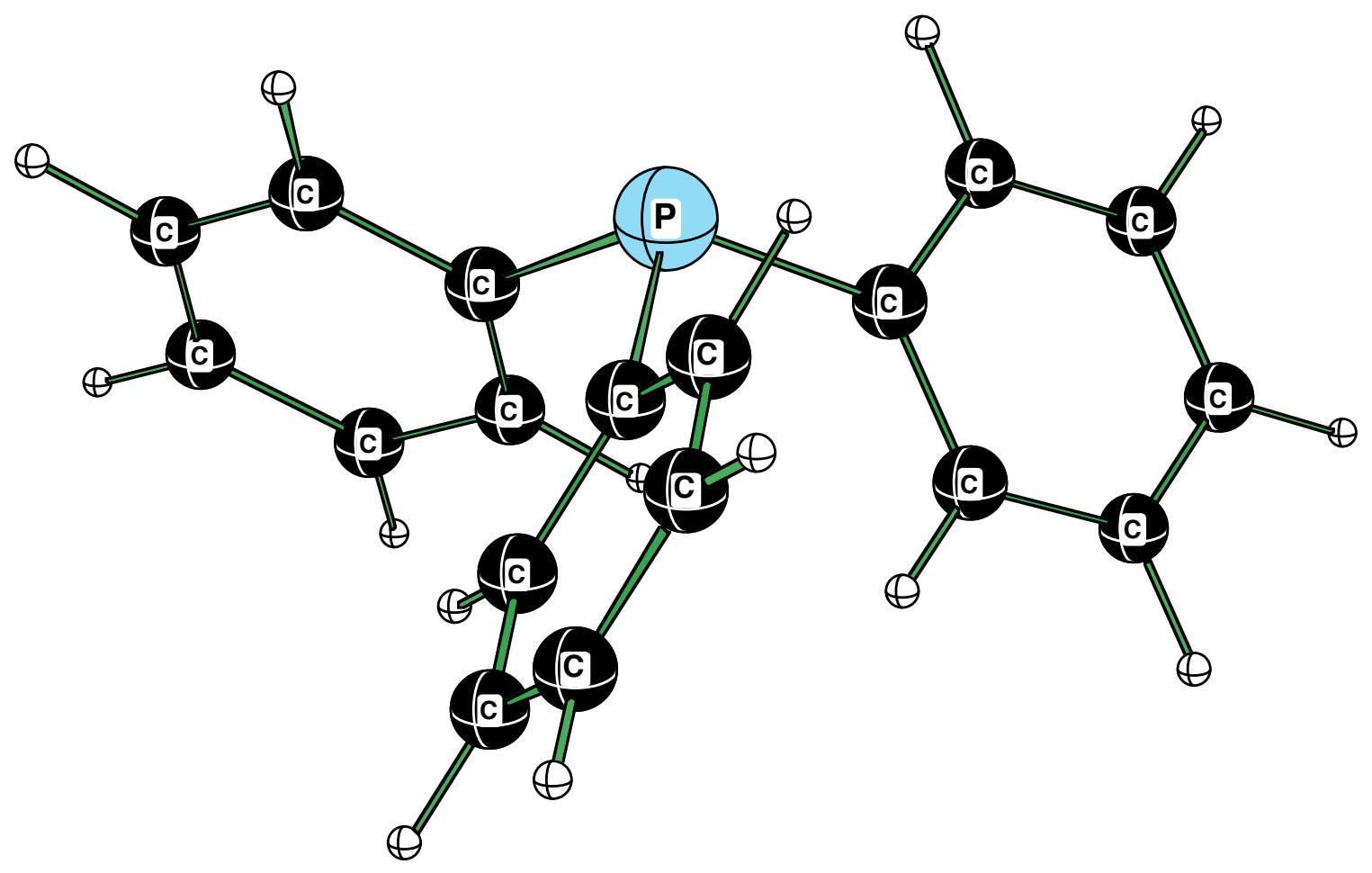




$\begin{array}{lccc}\text { Coordinates } \\ 15 & -0.217811 & -0.026258 & -0.014396 \\ 6 & 0.061912 & -0.021187 & 1.811311 \\ 6 & 1.287868 & -0.254543 & 2.429380 \\ 6 & 1.396622 & -0.248145 & 3.811539 \\ 6 & 0.282860 & -0.005867 & 4.598256 \\ 6 & -0.944601 & 0.223122 & 3.997549 \\ 6 & -1.053009 & 0.206104 & 2.617355 \\ 1 & 2.161738 & -0.446799 & 1.835732 \\ 1 & 2.351408 & -0.432799 & 4.271254 \\ 1 & 0.369090 & -0.001887 & 5.670317 \\ 1 & -1.816317 & 0.404417 & 4.600812 \\ 1 & -2.014216 & 0.366960 & 2.161308 \\ 6 & 1.278377 & -0.886567 & -0.672272 \\ 6 & 1.195022 & -2.271116 & -0.815563 \\ 6 & 2.262773 & -3.003689 & -1.305652 \\ 6 & 3.430953 & -2.359019 & -1.679465 \\ 6 & 3.523672 & -0.982598 & -1.557298 \\ 6 & 2.456954 & -0.252045 & -1.056620 \\ 1 & 0.285915 & -2.779305 & -0.545282 \\ 1 & 2.179108 & -4.071367 & -1.404371 \\ 1 & 4.259302 & -2.923961 & -2.068623 \\ 1 & 4.425250 & -0.474905 & -1.851338 \\ 1 & 2.546155 & 0.814646 & -0.971531 \\ 6 & 0.059114 & 1.736058 & -0.492964 \\ 6 & 0.705195 & 2.683454 & 0.297233 \\ 6 & 0.850230 & 3.990845 & -0.140915 \\ 6 & 0.354494 & 4.371964 & -1.376653 \\ 6 & -0.295004 & 3.441467 & -2.171832 \\ 6 & -0.447816 & 2.138953 & -1.727937 \\ 1 & 1.093741 & 2.406911 & 1.259307 \\ 1 & 1.350089 & 4.709429 & 0.484250 \\ 1 & 0.467453 & 5.386765 & -1.714568 \\ 1 & -0.689985 & 3.730485 & -3.129507 \\ 1 & -0.969780 & 1.428728 & -2.345121 \\ \mathrm{E}(\mathrm{RHF})=-1031.09010472 & \\ \text { ZPE=184.29374 } & & \\ \text { PCM E(in MeNO2)=-1031.090819 } \\ 6 & & \\ 6\end{array}$

\section{Triphenylphosphonium Cation}




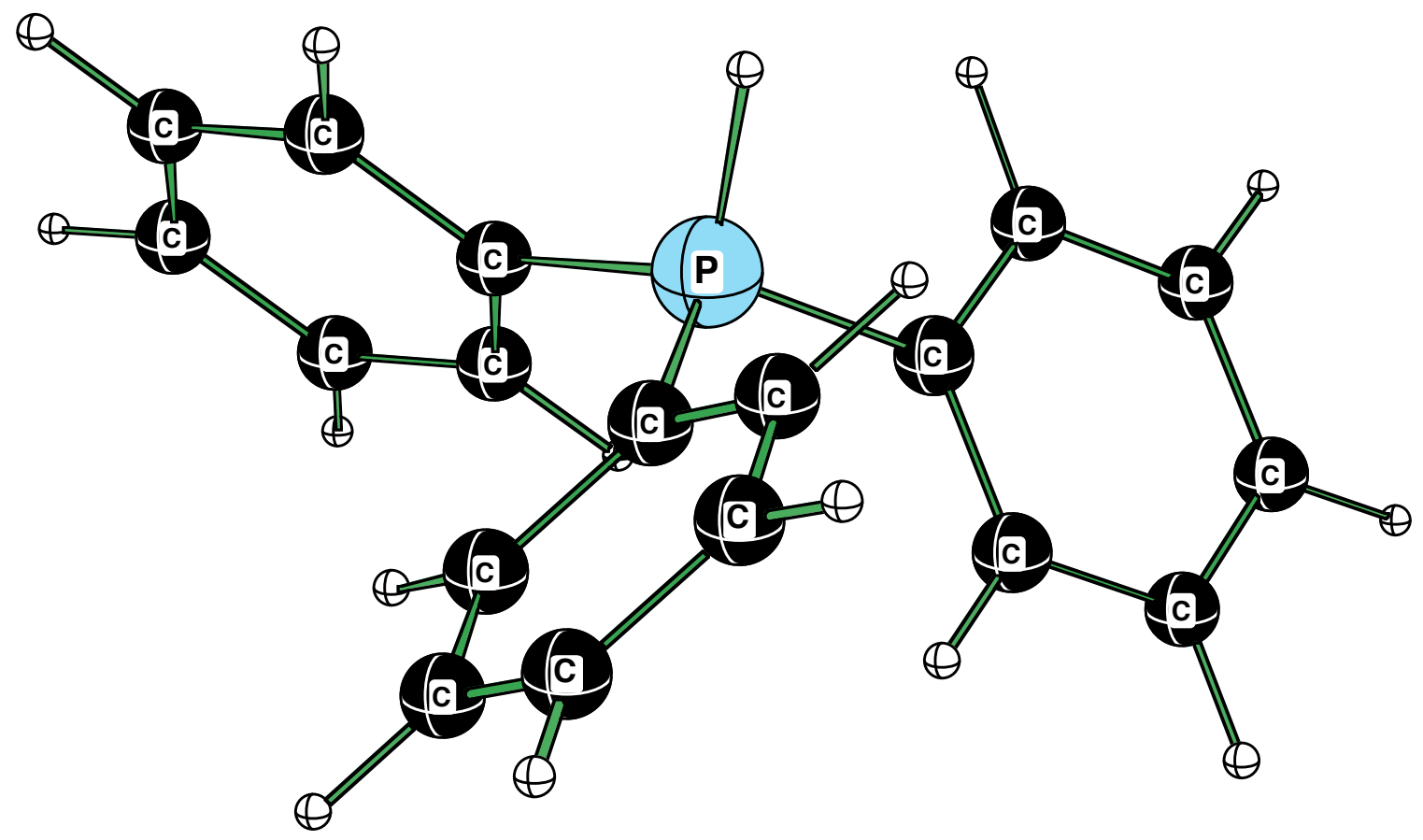

Coordinates

$\begin{array}{rrrr}15 & 0.289078 & 0.218086 & 0.169378 \\ 6 & 0.080076 & 0.044986 & 1.945528 \\ 6 & 1.177317 & -0.144970 & 2.781015 \\ 6 & 0.985513 & -0.256285 & 4.148325 \\ 6 & -0.292891 & -0.184678 & 4.676122 \\ 6 & -1.390400 & 0.000357 & 3.844778 \\ 6 & -1.207857 & 0.116027 & 2.481336 \\ 1 & 2.169964 & -0.212079 & 2.376384 \\ 1 & 1.830238 & -0.403322 & 4.794936 \\ 1 & -0.438176 & -0.276556 & 5.736678 \\ 1 & -2.380051 & 0.050161 & 4.258730 \\ 1 & -2.062130 & 0.259690 & 1.843017 \\ 6 & 1.846533 & -0.468623 & -0.406056 \\ 6 & 1.996742 & -1.856775 & -0.443175 \\ 6 & 3.189906 & -2.406024 & -0.867866 \\ 6 & 4.234461 & -1.575800 & -1.254557 \\ 6 & 4.088889 & -0.198988 & -1.219709 \\ 6 & 2.893766 & 0.360448 & -0.798487 \\ 1 & 1.191789 & -2.505430 & -0.144564 \\ 1 & 3.306335 & -3.473055 & -0.901559 \\ 1 & 5.161203 & -2.006090 & -1.586712 \\ 1 & 4.898105 & 0.438377 & -1.523388 \\ 1 & 2.782982 & 1.428666 & -0.783823 \\ 6 & 0.039811 & 1.908250 & -0.387007 \\ 6 & 0.157642 & 2.978059 & 0.495990 \\ 6 & -0.021981 & 4.271063 & 0.032748 \\ 6 & -0.323105 & 4.493106 & -1.300684\end{array}$




$\begin{array}{lccc}6 & -0.445786 & 3.427528 & -2.183512 \\ 6 & -0.264668 & 2.135973 & -1.731008 \\ 1 & 0.380350 & 2.811306 & 1.533377 \\ 1 & 0.067921 & 5.098019 & 0.711972 \\ 1 & -0.466749 & 5.497104 & -1.655202 \\ 1 & -0.684366 & 3.604939 & -3.215428 \\ 1 & -0.360628 & 1.315113 & -2.420104 \\ 1 & -0.710840 & -0.537276 & -0.416765 \\ \text { E(RHF) }=-1031.48492703 \\ \text { ZPE=191.73927 } \\ \text { PCM E(in MeNO2)=-1031.545021 }\end{array}$

\section{Tricyclohexylphosphine}

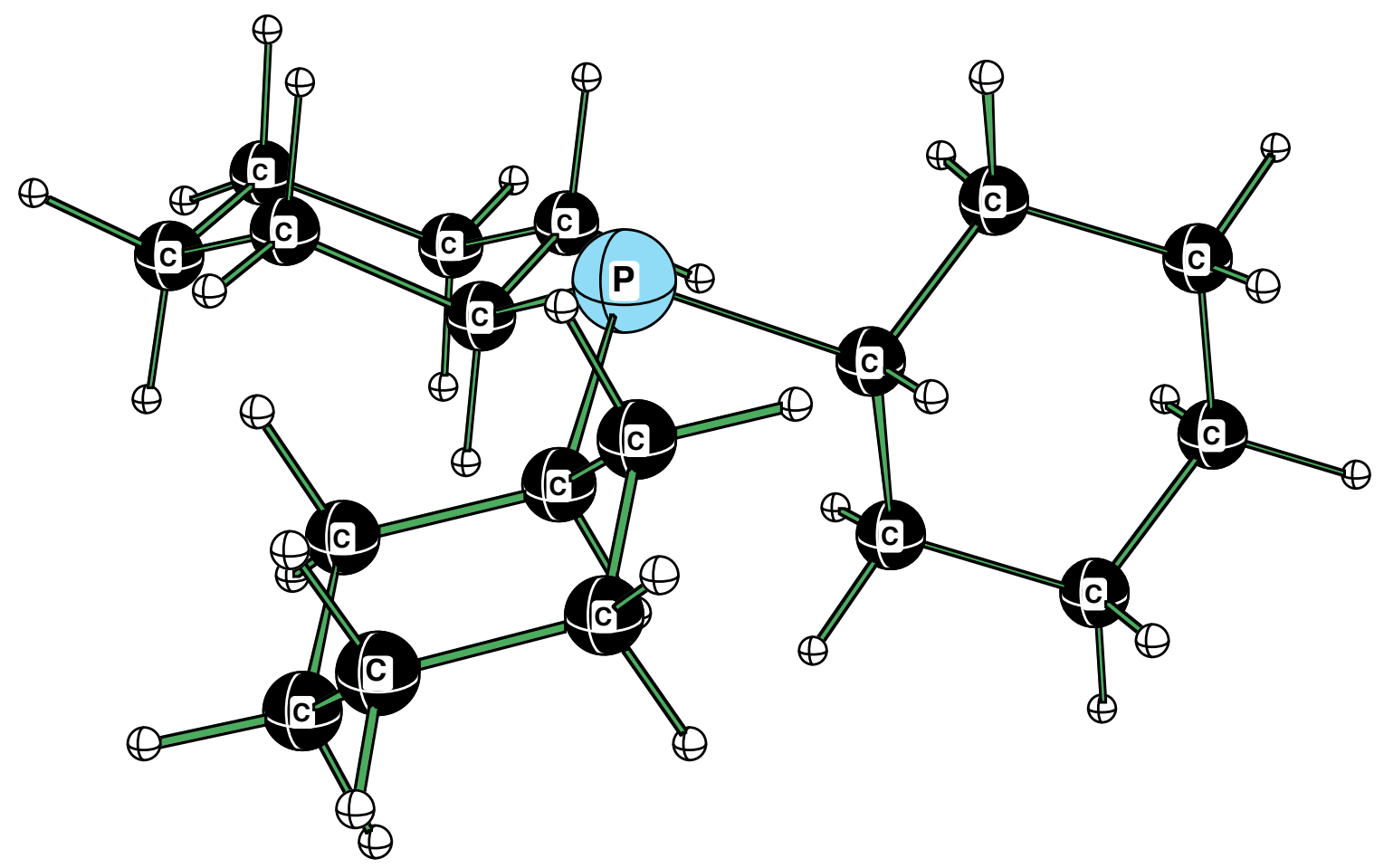

Coordinates

$\begin{array}{crrr}15 & 0.115506 & 0.044650 & -0.881471 \\ 6 & -1.455399 & -3.159705 & 1.514227 \\ 6 & -1.018318 & -1.726317 & 1.186032 \\ 6 & -0.648597 & -1.581096 & -0.302082 \\ 6 & -1.808477 & -2.045387 & -1.204134 \\ 6 & -2.248635 & -3.473299 & -0.862061 \\ 6 & -2.608969 & -3.615909 & 0.618309 \\ 1 & -1.827424 & -1.050547 & 1.443308 \\ 1 & -0.179776 & -1.453109 & 1.818200 \\ 1 & -0.609820 & -3.833063 & 1.382522\end{array}$




\begin{tabular}{|c|c|c|c|}
\hline 1 & -1.743975 & -3.225352 & 2.560277 \\
\hline 1 & -2.660355 & -1.379763 & -1.099635 \\
\hline 1 & -1.503464 & -1.992413 & -2.244477 \\
\hline 1 & -3.094478 & -3.756732 & -1.483088 \\
\hline 1 & -1.442065 & -4.164378 & -1.102542 \\
\hline 1 & -3.490762 & -3.014039 & 0.833947 \\
\hline 1 & -2.873961 & -4.645596 & 0.843148 \\
\hline 1 & 0.164335 & -2.282186 & -0.486025 \\
\hline 6 & -1.040296 & 3.965267 & 0.164455 \\
\hline 6 & -0.336183 & 2.795916 & -0.531575 \\
\hline 6 & -0.789528 & 1.427532 & 0.024780 \\
\hline 6 & -2.319501 & 1.335012 & -0.148553 \\
\hline 6 & -3.046721 & 2.495121 & 0.542082 \\
\hline 6 & -2.559925 & 3.855362 & 0.043453 \\
\hline 1 & -0.560676 & 2.825295 & -1.595701 \\
\hline 1 & 0.733755 & 2.923478 & -0.445281 \\
\hline 1 & -0.760148 & 3.981539 & 1.216771 \\
\hline 1 & -0.696372 & 4.903993 & -0.262167 \\
\hline 1 & -2.558365 & 1.343610 & -1.210715 \\
\hline 1 & -2.703084 & 0.404891 & 0.245877 \\
\hline 1 & -4.117605 & 2.397788 & 0.382793 \\
\hline 1 & -2.885852 & 2.426343 & 1.616913 \\
\hline 1 & -2.845993 & 3.979204 & -0.999730 \\
\hline 1 & -3.044410 & 4.655543 & 0.596680 \\
\hline 1 & -0.565348 & 1.387846 & 1.089790 \\
\hline 6 & 4.058154 & -1.275519 & -0.242675 \\
\hline 6 & 2.711123 & -0.984900 & -0.911686 \\
\hline 6 & 1.786701 & -0.137415 & -0.012575 \\
\hline 6 & 2.510429 & 1.164286 & 0.368656 \\
\hline 6 & 3.858427 & 0.881557 & 1.043705 \\
\hline 6 & 4.765945 & 0.016093 & 0.168711 \\
\hline 1 & 2.879069 & -0.450141 & -1.843860 \\
\hline 1 & 2.234471 & -1.922218 & -1.182012 \\
\hline 1 & 3.898396 & -1.896428 & 0.637555 \\
\hline 1 & 4.686934 & -1.850198 & -0.917777 \\
\hline 1 & 2.680830 & 1.766299 & -0.521389 \\
\hline 1 & 1.900331 & 1.756659 & 1.041319 \\
\hline 1 & 4.350498 & 1.821419 & 1.280527 \\
\hline 1 & 3.683779 & 0.375676 & 1.992042 \\
\hline 1 & 5.042424 & 0.573869 & -0.724615 \\
\hline 1 & 5.690517 & -0.209426 & 0.693391 \\
\hline 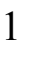 & 1.624636 & -0.687216 & 0.911609 \\
\hline \multicolumn{4}{|c|}{$\mathrm{E}(\mathrm{RHF})=-1041.58119495$} \\
\hline \multicolumn{4}{|c|}{$\mathrm{ZPE}=325.72359$} \\
\hline
\end{tabular}

Phosnhines SI v1.1 


\section{Tricyclohexylphosphonium Cation}

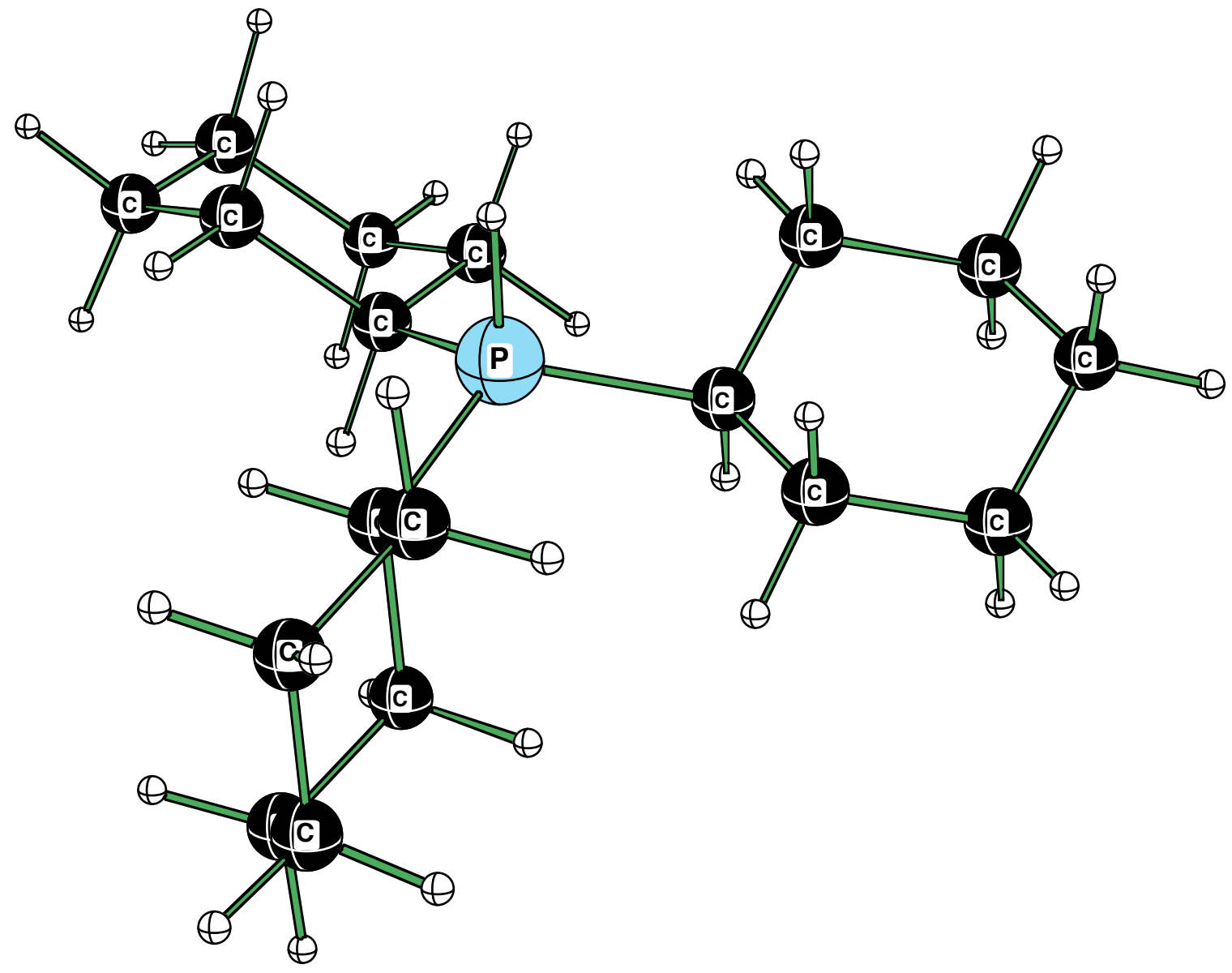

Coordinates

$\begin{array}{rrrr}15 & 0.108693 & -0.007350 & -0.504353 \\ 6 & -1.896104 & -3.179347 & 1.386982 \\ 6 & -1.245667 & -1.798627 & 1.232820 \\ 6 & -0.856925 & -1.557157 & -0.241686 \\ 6 & -2.064297 & -1.711369 & -1.189732 \\ 6 & -2.701335 & -3.094412 & -1.008258 \\ 6 & -3.092807 & -3.349287 & 0.448835 \\ 1 & -1.943612 & -1.037773 & 1.565596 \\ 1 & -0.375553 & -1.733847 & 1.879292 \\ 1 & -1.156073 & -3.948887 & 1.179340 \\ 1 & -2.202357 & -3.312378 & 2.418854 \\ 1 & -2.806360 & -0.948555 & -0.983811 \\ 1 & -1.751814 & -1.583273 & -2.221944 \\ 1 & -3.570885 & -3.170525 & -1.651892 \\ 1 & -2.001412 & -3.858768 & -1.338355 \\ 1 & -3.882055 & -2.658671 & 0.738802 \\ 1 & -3.502108 & -4.348138 & 0.551865 \\ 1 & -0.137119 & -2.325070 & -0.516131\end{array}$




$\begin{array}{cccc}6 & -0.562491 & 4.090995 & -0.034318 \\ 6 & -0.026069 & 2.774957 & -0.608888 \\ 6 & -0.627848 & 1.560741 & 0.139937 \\ 6 & -2.168612 & 1.609667 & 0.049518 \\ 6 & -2.699967 & 2.929973 & 0.621588 \\ 6 & -2.089200 & 4.144138 & -0.077317 \\ 1 & -0.296247 & 2.707347 & -1.660295 \\ 1 & 1.054276 & 2.775720 & -0.559372 \\ 1 & -0.218271 & 4.200978 & 0.991596 \\ 1 & -0.138117 & 4.916713 & -0.595081 \\ 1 & -2.474025 & 1.520588 & -0.990160 \\ 1 & -2.616892 & 0.785516 & 0.587508 \\ 1 & -3.780376 & 2.942190 & 0.526700 \\ 1 & -2.480483 & 2.970267 & 1.686238 \\ 1 & -2.421917 & 4.172876 & -1.112616 \\ 1 & -2.442993 & 5.057149 & 0.388601 \\ 1 & -0.340761 & 1.608670 & 1.187643 \\ 6 & 3.936683 & -1.663848 & -0.384443 \\ 6 & 2.576413 & -1.224010 & -0.938335 \\ 6 & 1.824830 & -0.352362 & 0.094613 \\ 6 & 2.675791 & 0.872835 & 0.478999 \\ 6 & 4.028276 & 0.418627 & 1.041800 \\ 6 & 4.784047 & -0.466986 & 0.049870 \\ 1 & 2.725153 & -0.646736 & -1.847724 \\ 1 & 1.998462 & -2.101103 & -1.211933 \\ 1 & 3.779660 & -2.329313 & 0.461408 \\ 1 & 4.456260 & -2.240447 & -1.141952 \\ 1 & 2.849160 & 1.489363 & -0.397916 \\ 1 & 2.164255 & 1.486532 & 1.212475 \\ 1 & 4.617899 & 1.294135 & 1.290986 \\ 1 & 3.867136 & -0.123323 & 1.971116 \\ 1 & 5.056536 & 0.120159 & -0.824438 \\ 1 & 5.711419 & -0.812261 & 0.493265 \\ 1 & 1.670556 & -0.943716 & 0.994282 \\ 1 & 0.189961 & 0.157658 & -1.878552 \\ \mathrm{E}(\mathrm{RHF})=-1041.98812279 & \\ \mathrm{ZPE}=333.36448 & & \\ \mathrm{PCM} \mathrm{E}(\mathrm{in} \mathrm{MeNO} 2)=-1042.03979 \\ & & & \end{array}$

Isopropylphosphine 
Coordinates

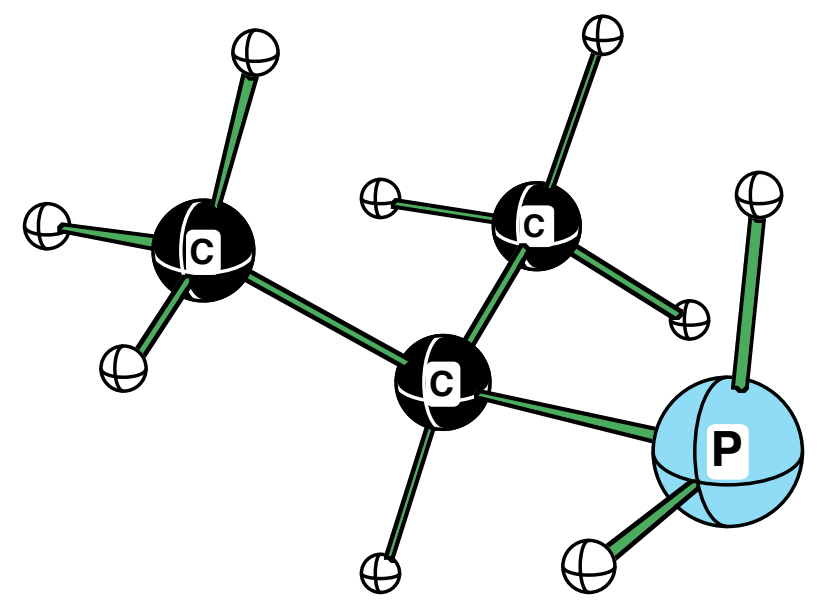

$$
\begin{array}{cccc}
15 & -0.073644 & 0.122892 & -0.064089 \\
1 & 0.075490 & -0.146081 & 1.307938 \\
1 & 1.256546 & -0.182384 & -0.396593 \\
6 & -0.873725 & -1.490025 & -0.574716 \\
1 & -0.867495 & -1.473109 & -1.662431 \\
6 & -0.128065 & -2.742532 & -0.106010 \\
1 & -0.100962 & -2.797608 & 0.978304 \\
1 & -0.621990 & -3.639939 & -0.470791 \\
1 & 0.895095 & -2.757484 & -0.465733 \\
6 & -2.336951 & -1.507901 & -0.113036 \\
1 & -2.886391 & -0.650720 & -0.487323 \\
1 & -2.835808 & -2.405351 & -0.467023 \\
1 & -2.405683 & -1.501520 & 0.971508 \\
\mathrm{E}(\mathrm{RHF})=-459.552963743 & \\
\mathrm{ZPE}=75.21368 & \multicolumn{3}{l}{}
\end{array}
$$

\section{Isopropylphosphonium Cation}


Coordinates

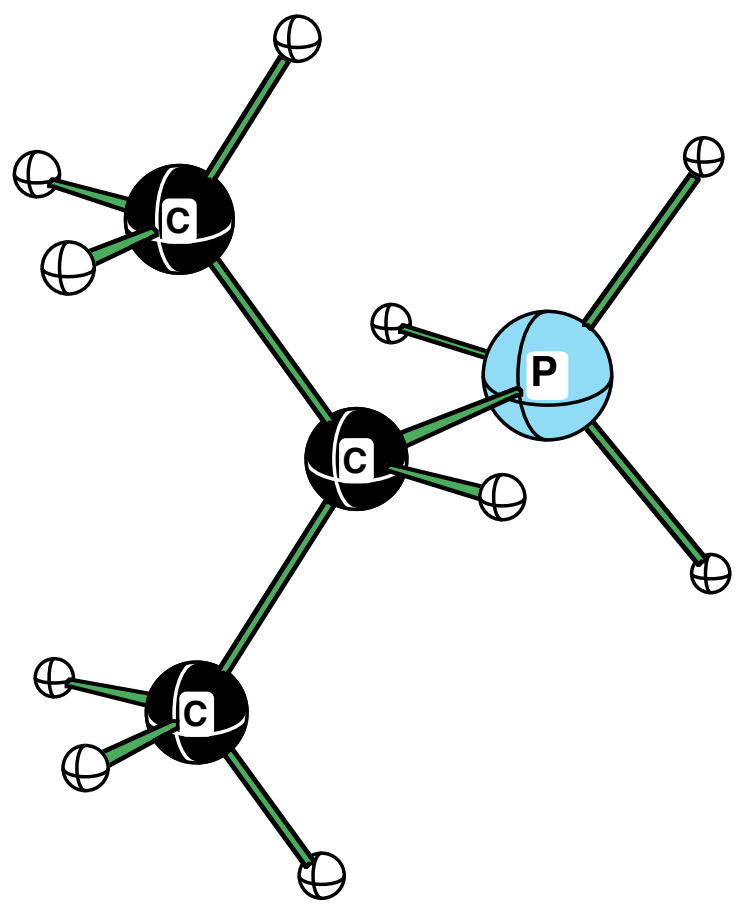

$$
\begin{array}{cccc}
15 & -0.014917 & -0.028050 & -0.020236 \\
1 & 0.031389 & 0.047730 & 1.358695 \\
1 & 1.292898 & 0.020159 & -0.462514 \\
6 & -0.888678 & -1.537736 & -0.583174 \\
1 & -0.859925 & -1.484678 & -1.667563 \\
6 & -0.128591 & -2.787740 & -0.108406 \\
1 & -0.116582 & -2.861790 & 0.973517 \\
1 & -0.636277 & -3.663826 & -0.492096 \\
1 & 0.891732 & -2.814912 & -0.473681 \\
6 & -2.349957 & -1.503577 & -0.104499 \\
1 & -2.883641 & -0.632410 & -0.467092 \\
1 & -2.856796 & -2.380173 & -0.488140 \\
1 & -2.417270 & -1.531712 & 0.977565 \\
1 & -0.626934 & 1.130052 & -0.459041 \\
\mathrm{E}(\mathrm{RHF})=-459.900276810 \\
\text { ZPE }=82.67097
\end{array}
$$

\section{Diisopropylphosphine}




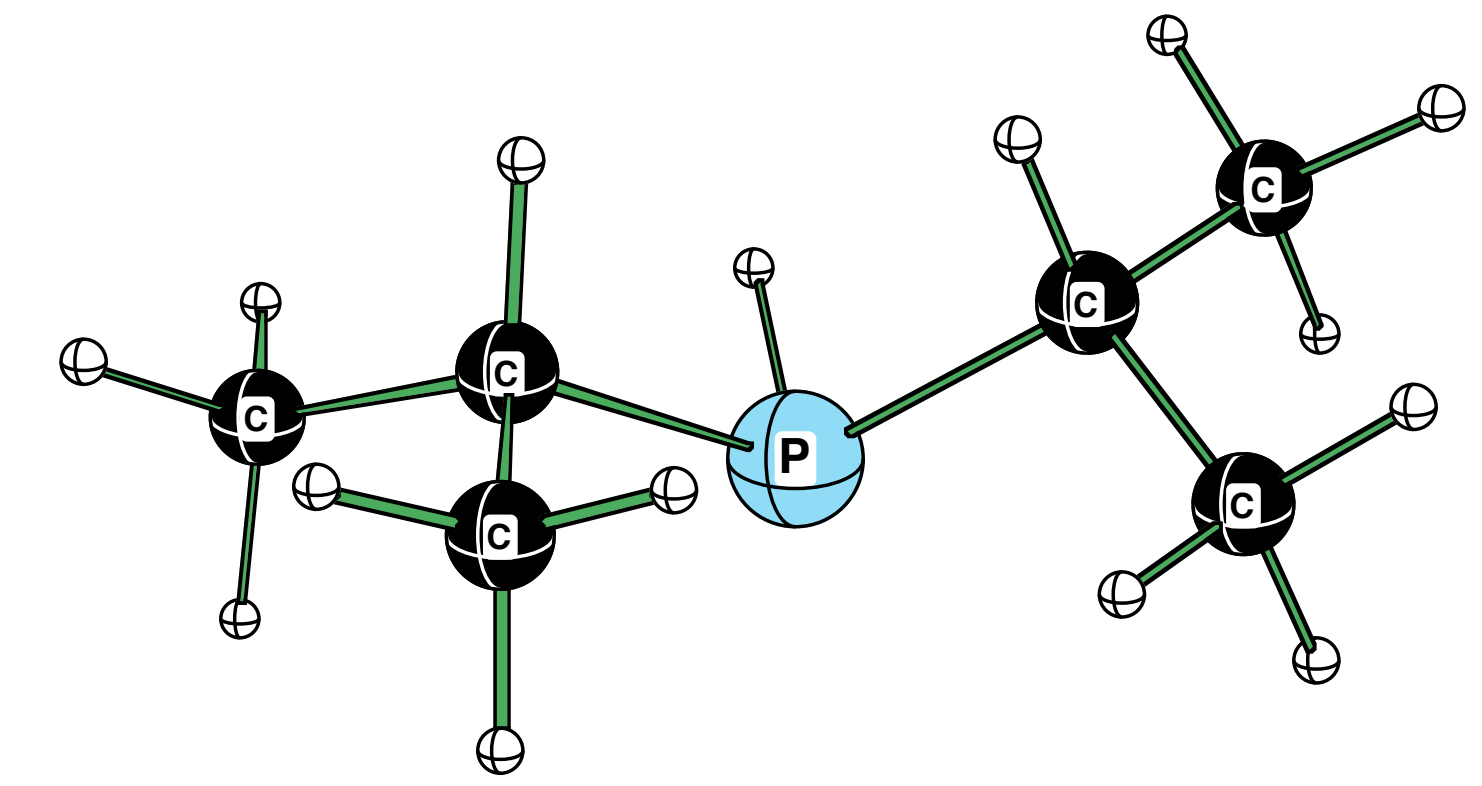

Coordinates

$\begin{array}{cccc}15 & -0.002789 & -0.014732 & 0.014249 \\ 1 & -0.040877 & 0.017586 & 1.418193 \\ 6 & 1.865714 & -0.010018 & -0.141027 \\ 1 & 2.254715 & 0.837715 & 0.417078 \\ 6 & 2.422226 & -1.295022 & 0.485871 \\ 1 & 2.056253 & -2.175809 & -0.033438 \\ 1 & 3.507207 & -1.304257 & 0.429694 \\ 1 & 2.145897 & -1.385775 & 1.531612 \\ 6 & 2.324500 & 0.119563 & -1.597804 \\ 1 & 2.034459 & 1.069402 & -2.033193 \\ 1 & 3.407326 & 0.053317 & -1.658585 \\ 1 & 1.908749 & -0.672790 & -2.213567 \\ 6 & -0.496348 & 1.772214 & -0.286162 \\ 1 & 0.361419 & 2.409062 & -0.084272 \\ 6 & -1.626779 & 2.173820 & 0.671009 \\ 1 & -1.306020 & 2.143917 & 1.706636 \\ 1 & -1.964425 & 3.183781 & 0.455782 \\ 1 & -2.484040 & 1.513876 & 0.567455 \\ 6 & -0.937503 & 1.987310 & -1.740443 \\ 1 & -1.188207 & 3.031068 & -1.909691 \\ 1 & -0.165536 & 1.716162 & -2.451767 \\ 1 & -1.815948 & 1.391513 & -1.968498 \\ \mathrm{E}(\mathrm{RHF})=-576.655554684 & \\ \mathrm{ZPE}=133.21547 & & \end{array}$

Diisopropylphosphonium Cation 


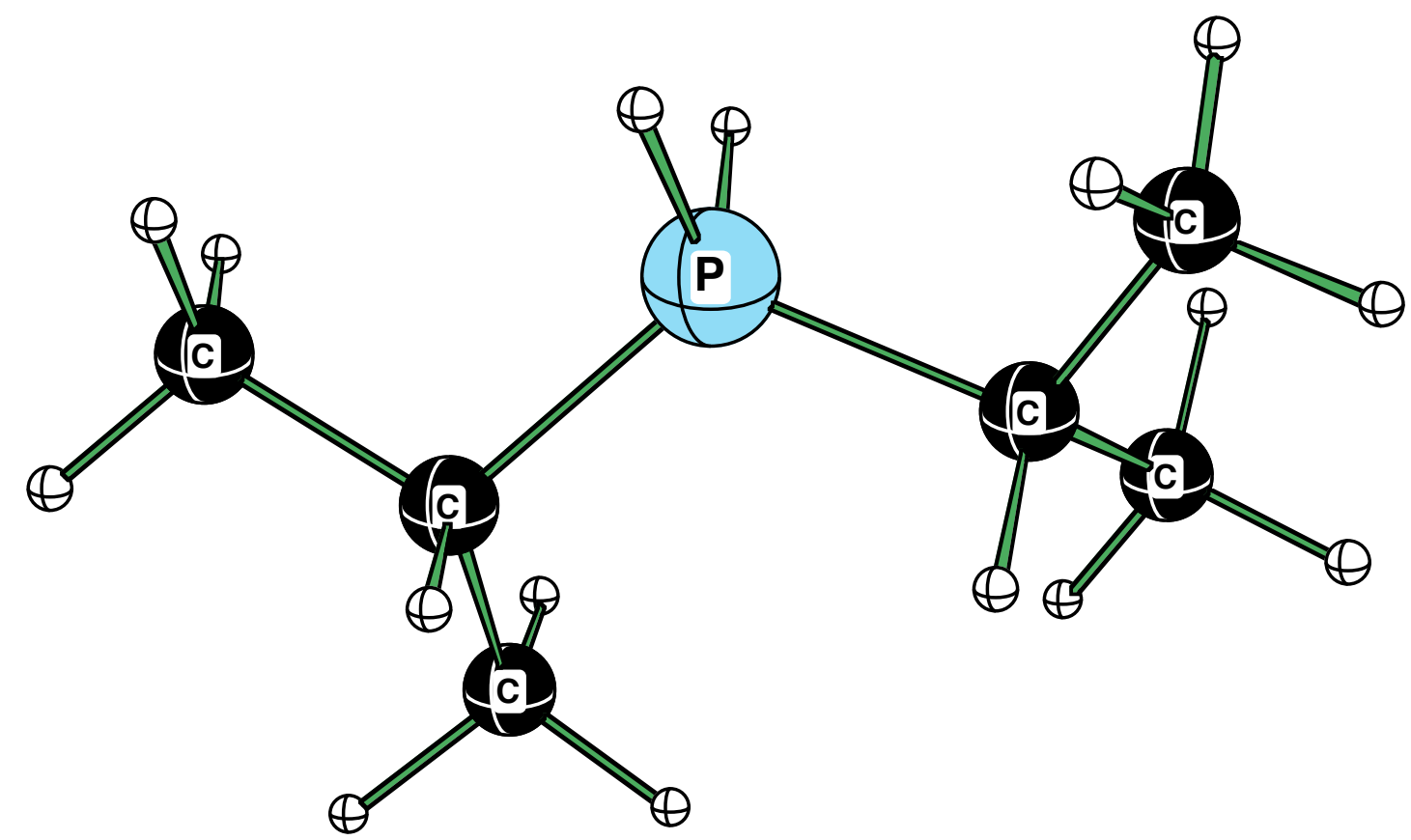

Coordinates

$\begin{array}{crrr}15 & 0.029055 & 0.035604 & -0.149033 \\ 1 & -0.010354 & -0.039756 & 1.231367 \\ 6 & 1.793724 & -0.010952 & -0.649480 \\ 1 & 2.232256 & 0.871153 & -0.190449 \\ 6 & 2.453985 & -1.267088 & -0.054235 \\ 1 & 2.039688 & -2.174505 & -0.480152 \\ 1 & 3.511599 & -1.247896 & -0.286575 \\ 1 & 2.357706 & -1.313937 & 1.024747 \\ 6 & 1.971639 & 0.073797 & -2.173199 \\ 1 & 1.594014 & 1.002838 & -2.582087 \\ 1 & 3.029521 & 0.026292 & -2.400143 \\ 1 & 1.489969 & -0.754836 & -2.681320 \\ 6 & -0.968382 & 1.483280 & -0.691220 \\ 1 & -0.270130 & 2.314302 & -0.731427 \\ 6 & -2.055391 & 1.789908 & 0.353964 \\ 1 & -1.636160 & 2.028402 & 1.324426 \\ 1 & -2.626466 & 2.647862 & 0.021418 \\ 1 & -2.746266 & 0.960969 & 0.468423 \\ 6 & -1.569745 & 1.252030 & -2.088547 \\ 1 & -2.090677 & 2.150848 & -2.394246 \\ 1 & -0.821160 & 1.036922 & -2.840871 \\ 1 & -2.289571 & 0.441052 & -2.076439 \\ 1 & -0.592514 & -1.124241 & -0.579571 \\ \mathrm{E}(\mathrm{RHF})=-577.028758989 & \\ \mathrm{ZPE}=140.59726 & \end{array}$

Triisopropylphosphine 


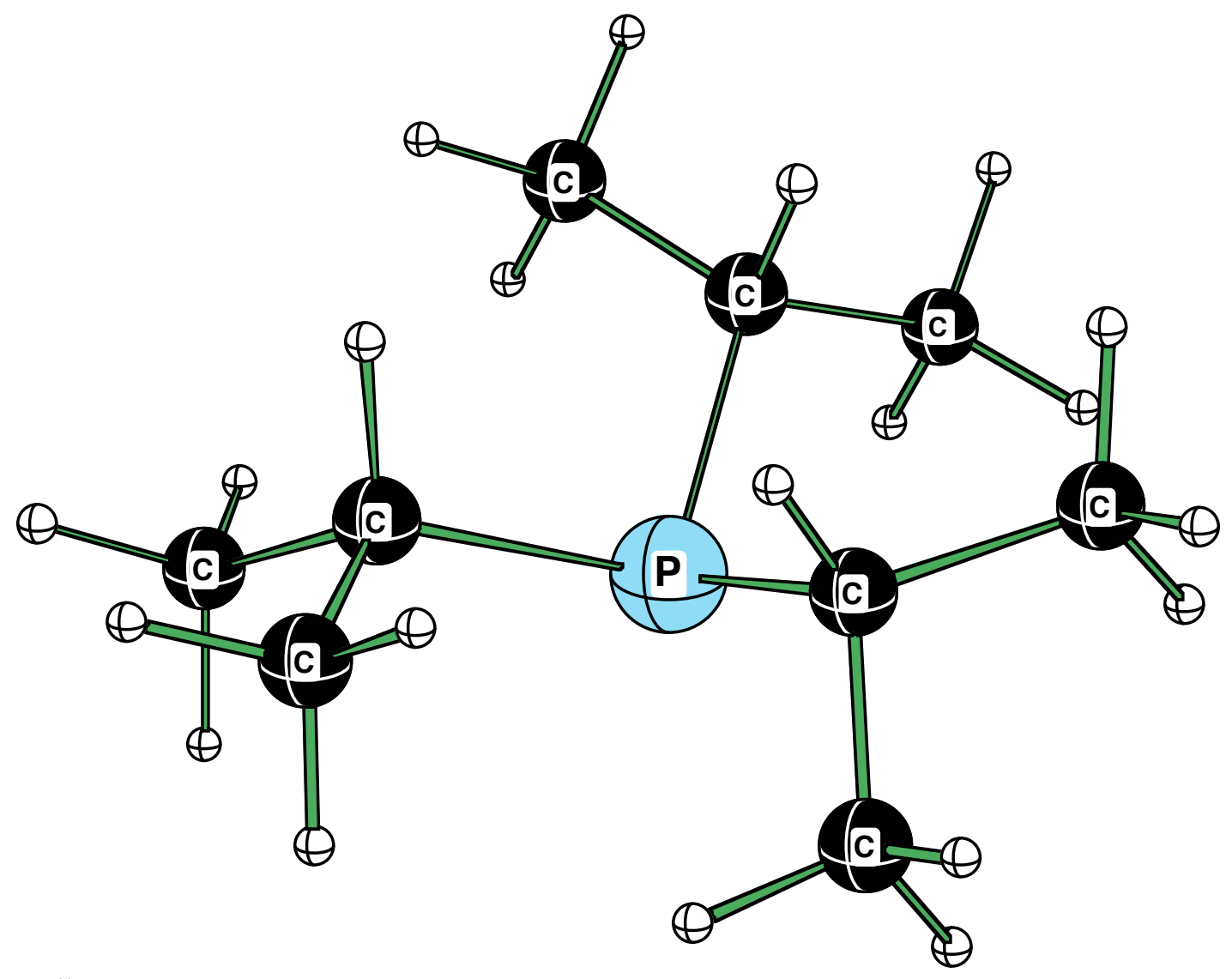

Coordinates

$\begin{array}{cccc}15 & -0.306832 & 0.049116 & -0.175089 \\ 6 & -0.146473 & 0.128148 & 1.709498 \\ 1 & 0.832228 & 0.536711 & 1.948459 \\ 6 & -1.230295 & 1.057516 & 2.281317 \\ 1 & -2.214972 & 0.629343 & 2.121909 \\ 1 & -1.090866 & 1.183852 & 3.351965 \\ 1 & -1.229129 & 2.041461 & 1.832809 \\ 6 & -0.258803 & -1.223667 & 2.428324 \\ 1 & 0.523649 & -1.918323 & 2.150838 \\ 1 & -0.182914 & -1.064277 & 3.500423 \\ 1 & -1.217404 & -1.696889 & 2.241101 \\ 6 & 0.725261 & -1.478690 & -0.604503 \\ 1 & 1.495863 & -1.590085 & 0.154232 \\ 6 & 1.435739 & -1.421593 & -1.964157 \\ 1 & 2.157992 & -0.618158 & -2.032274 \\ 1 & 1.972925 & -2.352318 & -2.124592 \\ 1 & 0.728181 & -1.314480 & -2.780117 \\ 6 & -0.172166 & -2.727564 & -0.593831 \\ 1 & 0.426174 & -3.621943 & -0.748054 \\ 1 & -0.717005 & -2.853926 & 0.331644 \\ 1 & -0.902075 & -2.672768 & -1.395429 \\ 6 & 0.816369 & 1.466147 & -0.735674\end{array}$




$\begin{array}{lccc}1 & 1.847566 & 1.130408 & -0.659352 \\ 6 & 0.511524 & 1.817798 & -2.201547 \\ 1 & 1.212679 & 2.566015 & -2.562448 \\ 1 & 0.570540 & 0.966628 & -2.865926 \\ 1 & -0.489080 & 2.229352 & -2.289280 \\ 6 & 0.698909 & 2.754211 & 0.091256 \\ 1 & -0.308593 & 3.156720 & 0.062142 \\ 1 & 0.982335 & 2.620872 & 1.127436 \\ 1 & 1.358656 & 3.508424 & -0.328827 \\ \mathrm{E}(\mathrm{RHF})=-693.745774869 \\ \mathrm{ZPE}=191.75596\end{array}$

Triisopropylphosphonium Cation

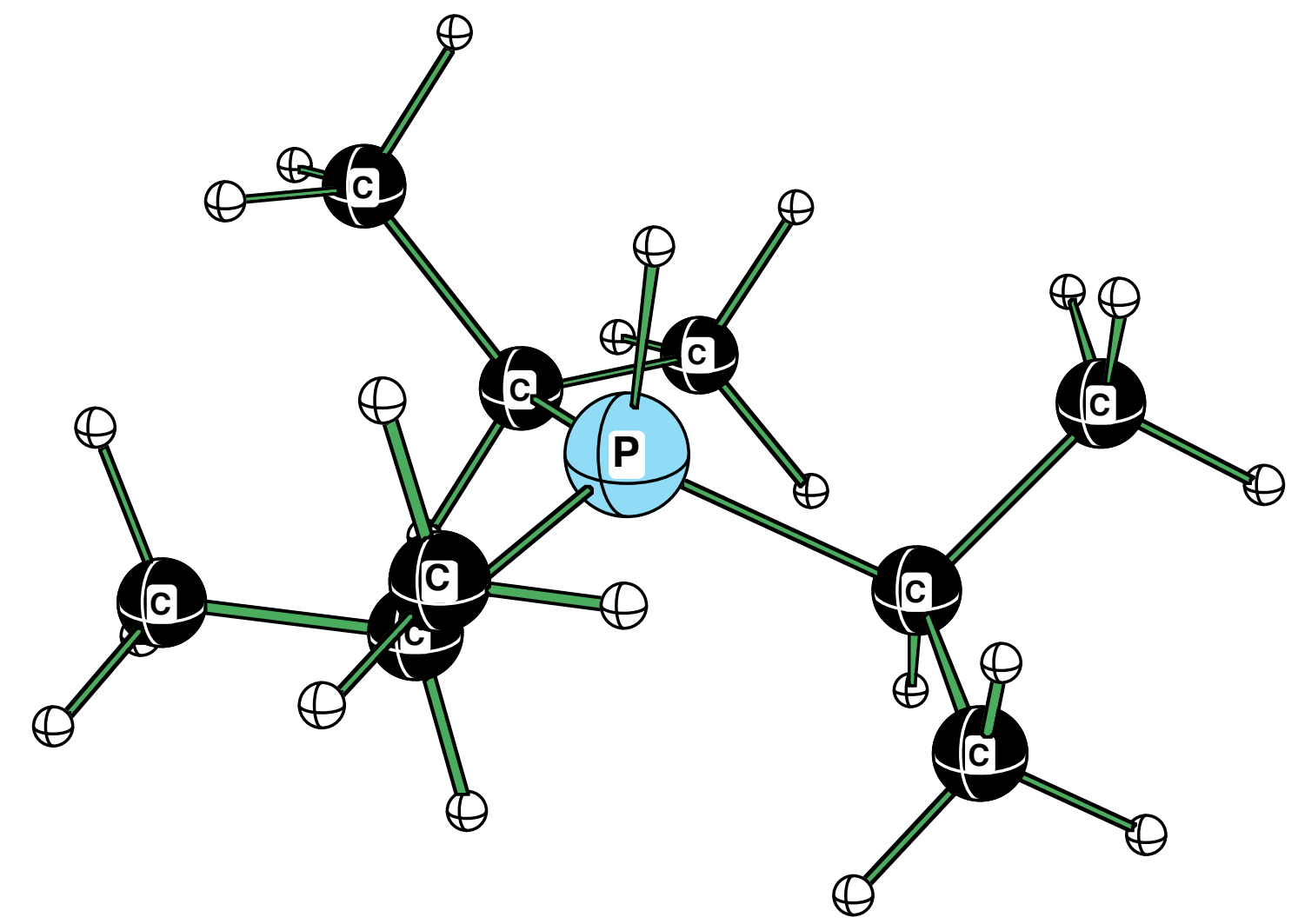

Coordinates

$\begin{array}{cccc}15 & 0.024064 & 0.041066 & -0.043608 \\ 6 & -0.116658 & 0.138544 & 1.802353 \\ 1 & 0.791483 & 0.643503 & 2.116222 \\ 6 & -1.334155 & 0.994583 & 2.198334 \\ 1 & -2.260004 & 0.497695 & 1.929644 \\ 1 & -1.332551 & 1.129222 & 3.273209 \\ 1 & -1.332036 & 1.977863 & 1.748111 \\ 6 & -0.172425 & -1.220097 & 2.519049 \\ 1 & 0.669927 & -1.859521 & 2.289052 \\ 1 & -0.153848 & -1.035138 & 3.586362\end{array}$




$$
\begin{array}{cccc}
1 & -1.088748 & -1.754416 & 2.300187 \\
6 & 0.799353 & -1.546610 & -0.604739 \\
1 & 1.532654 & -1.775187 & 0.162229 \\
6 & 1.535330 & -1.465776 & -1.951741 \\
1 & 2.302487 & -0.702711 & -1.974436 \\
1 & 2.023397 & -2.417753 & -2.122765 \\
1 & 0.851918 & -1.301073 & -2.775668 \\
6 & -0.255861 & -2.667678 & -0.646242 \\
1 & 0.238617 & -3.605727 & -0.867699 \\
1 & -0.785874 & -2.792890 & 0.288079 \\
1 & -0.983115 & -2.485259 & -1.429885 \\
1 & -1.270554 & 0.045113 & -0.539160 \\
6 & 0.868919 & 1.526600 & -0.762016 \\
1 & 1.915565 & 1.243635 & -0.813981 \\
6 & 0.346908 & 1.798493 & -2.185268 \\
1 & 0.929895 & 2.598664 & -2.624952 \\
1 & 0.424105 & 0.942405 & -2.841518 \\
1 & -0.688879 & 2.119318 & -2.162516 \\
6 & 0.766927 & 2.802444 & 0.089174 \\
1 & -0.247866 & 3.178436 & 0.132705 \\
1 & 1.136865 & 2.673758 & 1.098068 \\
1 & 1.374213 & 3.566537 & -0.380996 \\
\mathrm{E}(\mathrm{RHF})=-694.142700707 & \\
\mathrm{ZPE}=198.95658 & &
\end{array}
$$

\section{Benzylphosphine}

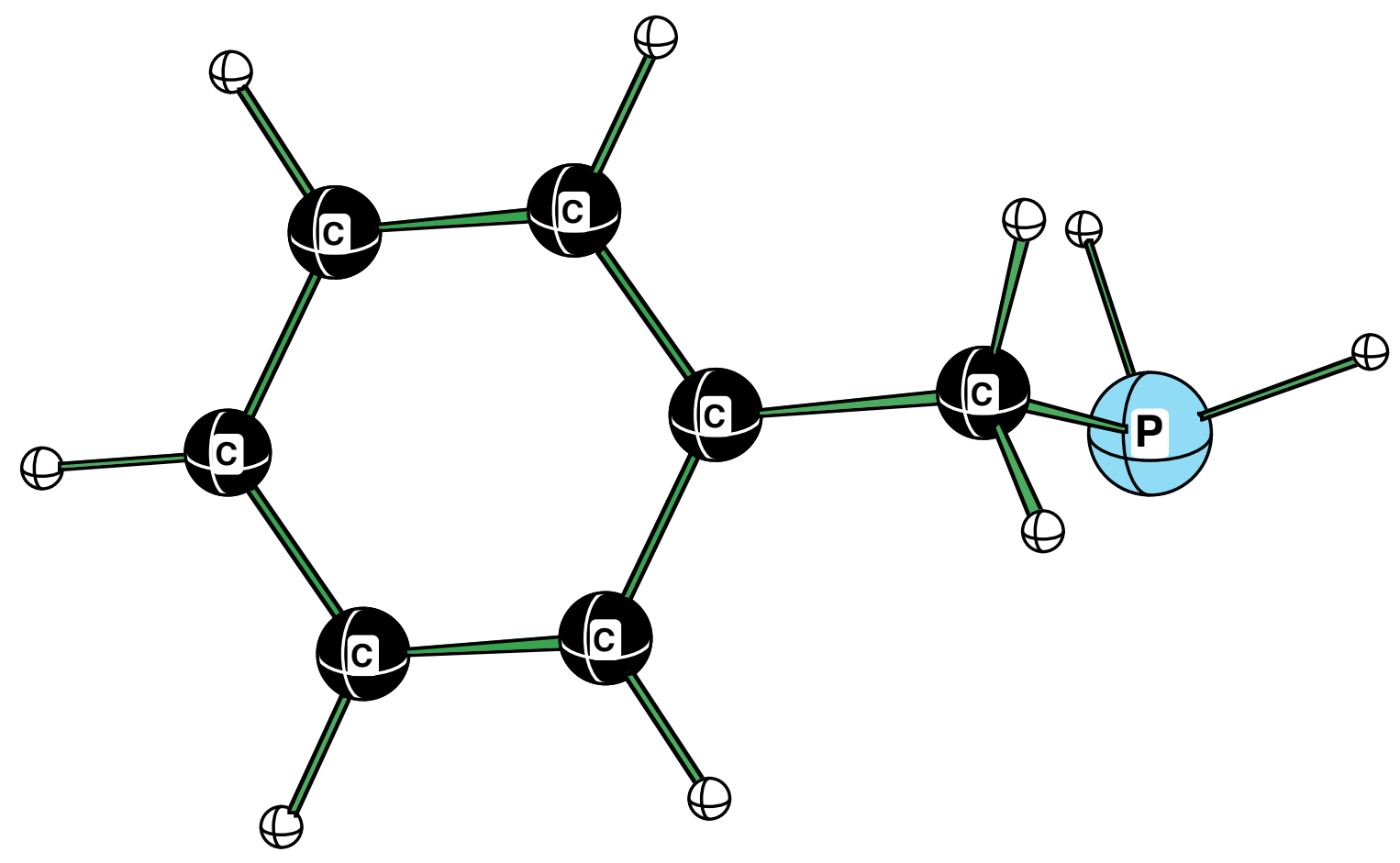




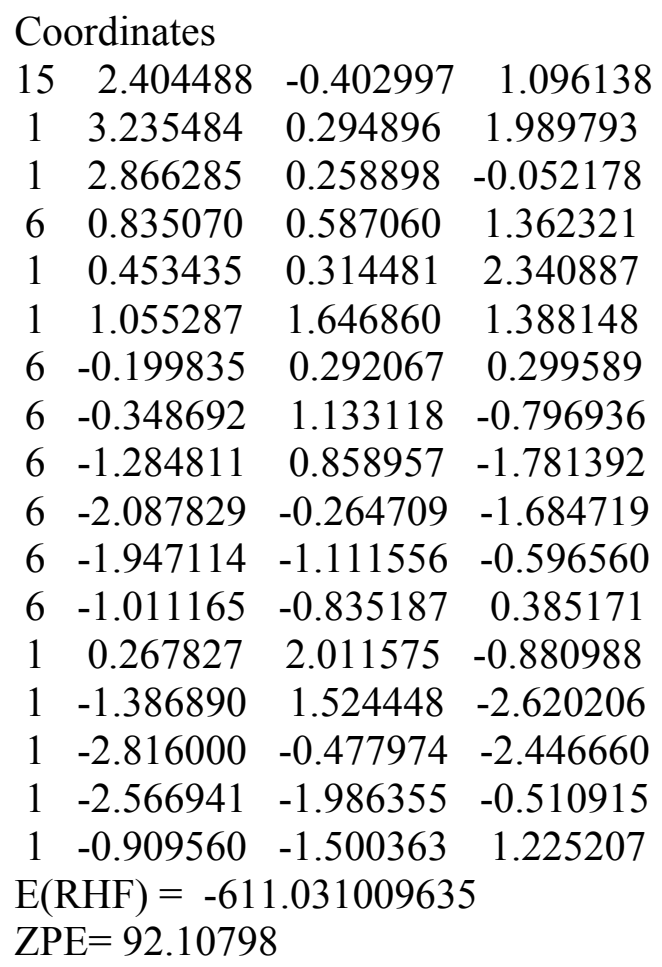

\section{Benzylphosphonium Cation}

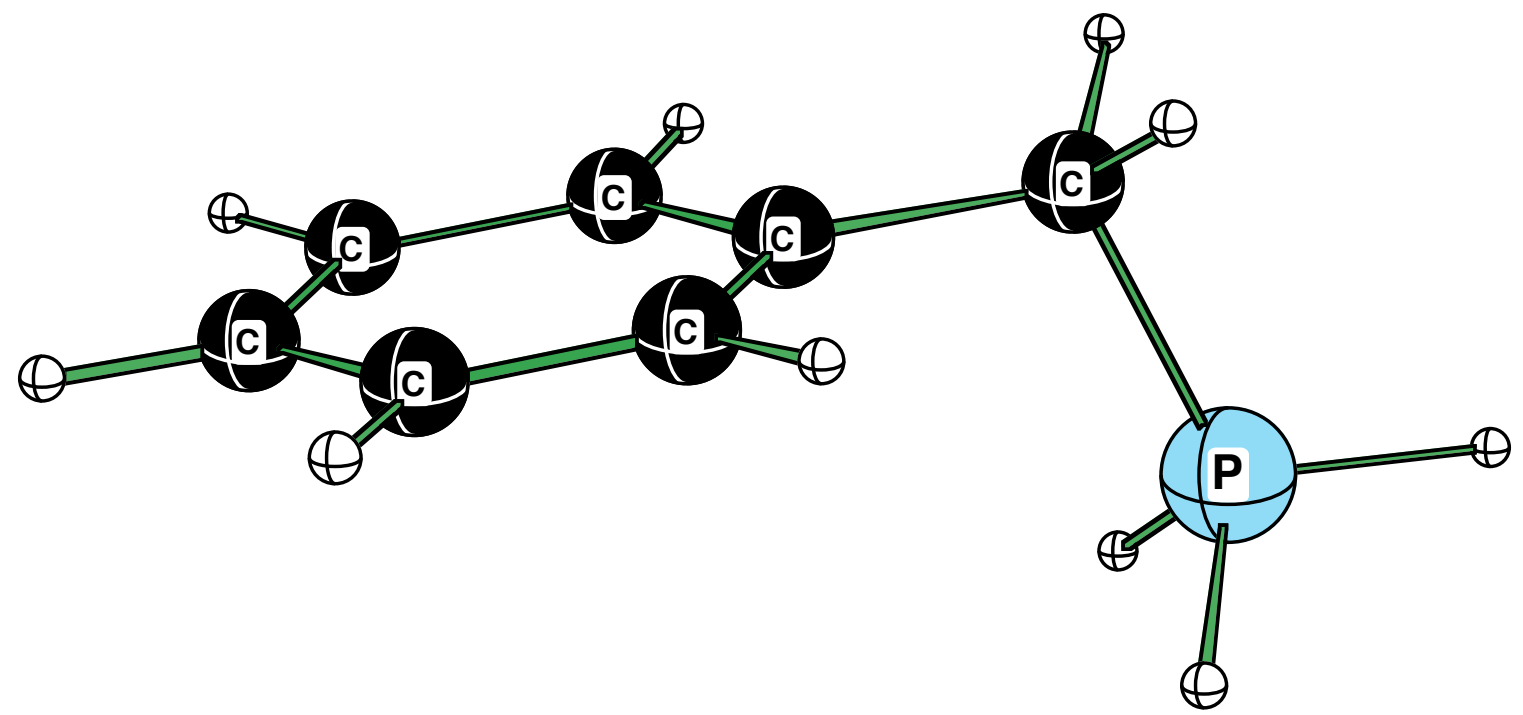

Coordinates

$\begin{array}{cccc}15 & 0.670637 & -1.103619 & 2.211069 \\ 1 & 0.700882 & -1.249072 & 3.586155 \\ 1 & 1.980608 & -0.998201 & 1.790966 \\ 6 & -0.317690 & 0.323391 & 1.641258 \\ 1 & -1.323039 & 0.194438 & 2.026154 \\ 1 & 0.101241 & 1.211092 & 2.101673 \\ 6 & -0.279512 & 0.382355 & 0.125613 \\ 6 & 0.718587 & 1.103679 & -0.519637\end{array}$




$$
\begin{array}{lccc}
6 & 0.756739 & 1.149984 & -1.902798 \\
6 & -0.199211 & 0.475317 & -2.646004 \\
6 & -1.196716 & -0.243649 & -2.006143 \\
6 & -1.237478 & -0.291848 & -0.623116 \\
1 & 1.453792 & 1.646649 & 0.049805 \\
1 & 1.523701 & 1.717035 & -2.396717 \\
1 & -0.171534 & 0.516092 & -3.719040 \\
1 & -1.945041 & -0.757635 & -2.580217 \\
1 & -2.027208 & -0.836865 & -0.134348 \\
1 & 0.178732 & -2.284631 & 1.694496 \\
\mathrm{E}(\mathrm{RHF})=-611.379419788 \\
\mathrm{ZPE}=99.61747
\end{array}
$$

\section{Dibenzylphosphine}

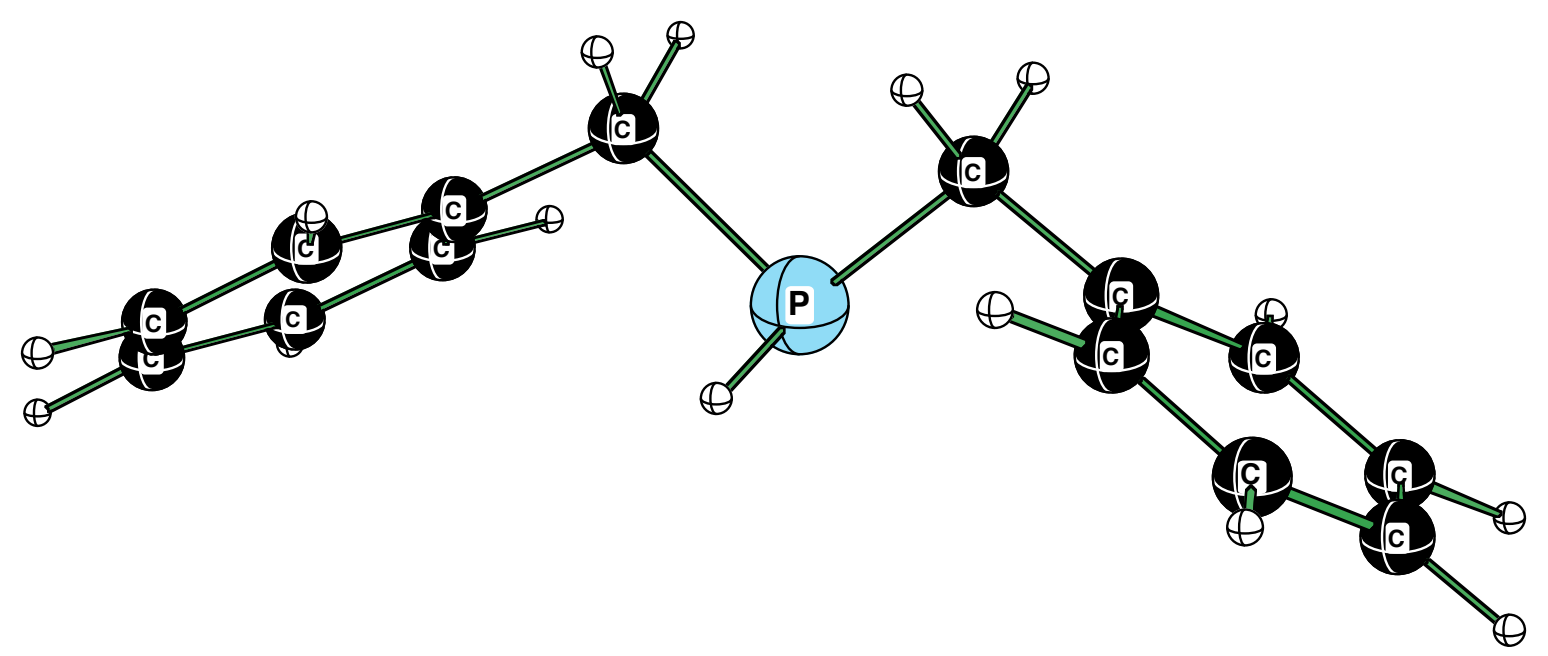

Coordinates

$\begin{array}{cccc}15 & -0.092573 & -0.067108 & -0.428597 \\ 1 & -0.116511 & -0.228592 & 0.965926 \\ 6 & 1.765527 & -0.090315 & -0.653922 \\ 1 & 1.965697 & 0.184789 & -1.685159 \\ 1 & 2.232002 & 0.662904 & -0.028200 \\ 6 & 2.353609 & -1.451705 & -0.357888 \\ 6 & 2.917594 & -1.726126 & 0.882712 \\ 6 & 3.442939 & -2.977140 & 1.164477 \\ 6 & 3.410936 & -3.977270 & 0.207598 \\ 6 & 2.849105 & -3.716208 & -1.032205 \\ 6 & 2.325045 & -2.465608 & -1.310706 \\ 1 & 2.951041 & -0.955487 & 1.633606 \\ 1 & 3.878152 & -3.167593 & 2.129521 \\ 1 & 3.819739 & -4.948063 & 0.423840 \\ 1 & 2.819770 & -4.485630 & -1.783207 \\ 1 & 1.889515 & -2.274564 & -2.276161 \\ 6 & -0.414733 & 1.776227 & -0.475211\end{array}$




$\begin{array}{lccc}1 & -0.271085 & 2.099738 & -1.501812 \\ 1 & 0.315761 & 2.303410 & 0.128880 \\ 6 & -1.814781 & 2.116903 & -0.016227 \\ 6 & -2.898207 & 2.006115 & -0.882595 \\ 6 & -4.181280 & 2.302641 & -0.455982 \\ 6 & -4.405488 & 2.714496 & 0.848251 \\ 6 & -3.335752 & 2.826174 & 1.720084 \\ 6 & -2.052387 & 2.528712 & 1.290078 \\ 1 & -2.735967 & 1.685414 & -1.897048 \\ 1 & -5.004945 & 2.213283 & -1.141887 \\ 1 & -5.401783 & 2.946636 & 1.179655 \\ 1 & -3.497403 & 3.146688 & 2.734053 \\ 1 & -1.228150 & 2.622319 & 1.976165 \\ \text { E(RHF) }=-879.615723509 & \\ \text { ZPE }=166.71539 & \end{array}$

Dibenzylphosphonium Cation

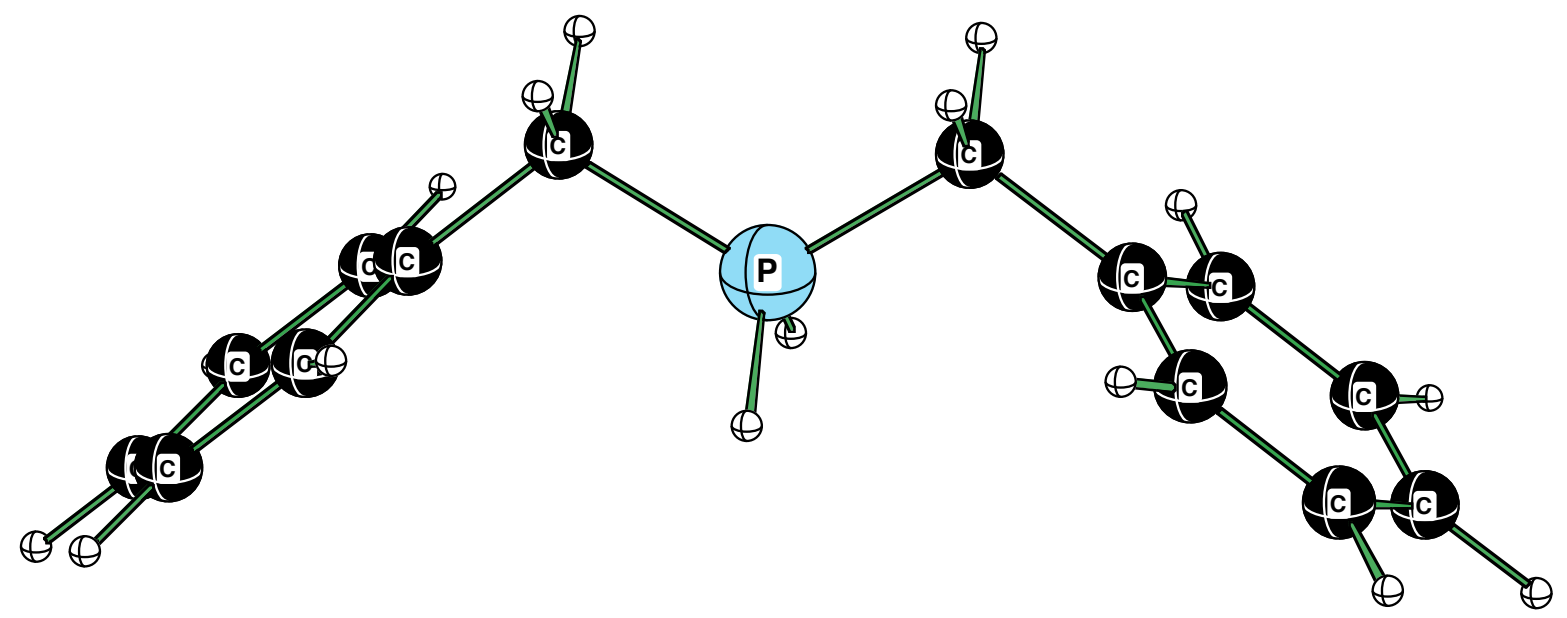

Coordinates

$\begin{array}{cccc}15 & 0.149114 & 0.224550 & -0.392296 \\ 1 & 0.387247 & 0.583160 & 0.919467 \\ 6 & 1.742060 & -0.176547 & -1.188377 \\ 1 & 1.533574 & -0.465850 & -2.212857 \\ 1 & 2.330394 & 0.734160 & -1.222981 \\ 6 & 2.447751 & -1.278464 & -0.420608 \\ 6 & 3.315591 & -0.962476 & 0.618377 \\ 6 & 3.949340 & -1.969217 & 1.326925 \\ 6 & 3.718435 & -3.296851 & 1.003377 \\ 6 & 2.855150 & -3.617073 & -0.032388 \\ 6 & 2.220494 & -2.611696 & -0.742061 \\ 1 & 3.514974 & 0.066365 & 0.865859 \\ 1 & 4.626008 & -1.717186 & 2.122335 \\ 1 & 4.214148 & -4.077258 & 1.550413 \\ 1 & 2.682010 & -4.644856 & -0.292694\end{array}$




\begin{tabular}{rrrr}
1 & 1.565118 & -2.870122 & -1.556439 \\
6 & -0.838563 & 1.537120 & -1.188383 \\
1 & -1.024308 & 1.232729 & -2.212866 \\
1 & -0.227438 & 2.432695 & -1.222976 \\
6 & -2.127963 & 1.760031 & -0.420629 \\
6 & -3.268599 & 1.033331 & -0.742099 \\
6 & -4.441461 & 1.228225 & -0.032440 \\
6 & -4.481255 & 2.148123 & 1.003330 \\
6 & -3.347196 & 2.876013 & 1.326896 \\
6 & -2.173427 & 2.682484 & 0.618361 \\
1 & -3.252511 & 0.329032 & -1.556480 \\
1 & -5.321568 & 0.669907 & -0.292760 \\
1 & -5.392862 & 2.302238 & 1.550355 \\
1 & -3.377436 & 3.597455 & 2.122308 \\
1 & -1.302526 & 3.265401 & 0.865857 \\
1 & -0.613676 & -0.924138 & -0.323782 \\
E(RHF) $=-879.990027644$ & \\
ZPE $=174.11413$ & \multicolumn{3}{l}{}
\end{tabular}

Tribenzylphosphine

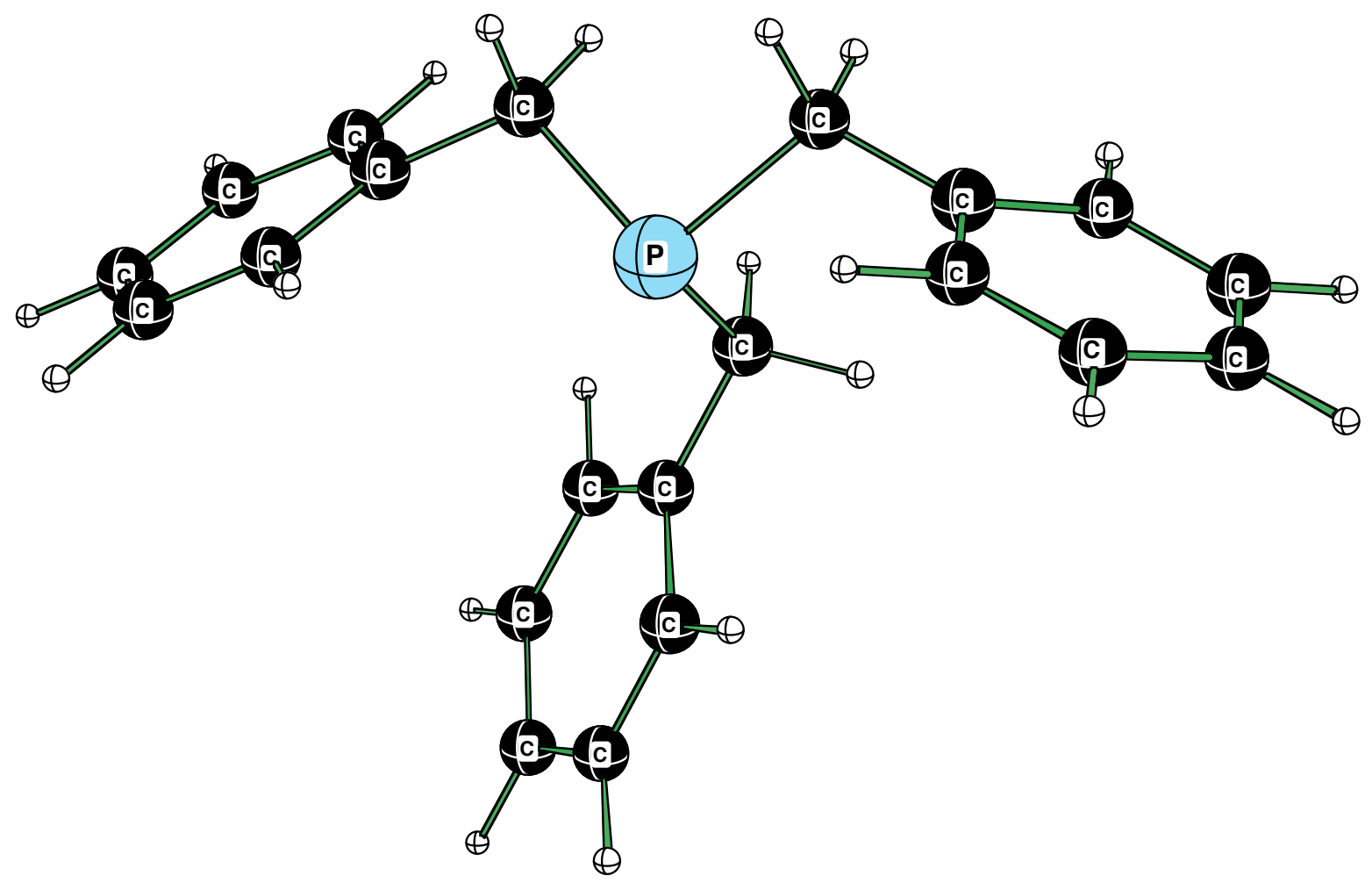

Coordinates
$\begin{array}{lllll}15 & -0.133174 & 0.323264 & -0.072329\end{array}$
$\begin{array}{llll}6 & -0.165985 & -0.067212 & 1.756766\end{array}$
$\begin{array}{lllll}1 & 0.847945 & -0.198441 & 2.123235\end{array}$
$\begin{array}{lllll}6 & 0.977319 & -1.091770 & -0.593301\end{array}$ 


$\begin{array}{cccc}1 & 1.899681 & -1.077407 & -0.019873 \\ 6 & 1.086354 & 1.738540 & -0.175335 \\ 1 & 1.826760 & 1.651995 & 0.614494 \\ 1 & -0.651427 & -1.037184 & 1.839103 \\ 1 & 0.455378 & -2.005267 & -0.320892 \\ 1 & 1.614047 & 1.600240 & -1.114003 \\ 6 & 1.290329 & -1.107856 & -2.073500 \\ 6 & 2.574318 & -0.845047 & -2.535380 \\ 6 & 0.296762 & -1.387328 & -3.009682 \\ 6 & 2.862176 & -0.859391 & -3.892487 \\ 1 & 3.359799 & -0.633069 & -1.830666 \\ 6 & 0.581241 & -1.404256 & -4.363232 \\ 1 & -0.705115 & -1.590818 & -2.674369 \\ 6 & 1.866984 & -1.139171 & -4.811460 \\ 1 & 3.863813 & -0.654383 & -4.226642 \\ 1 & -0.199922 & -1.624821 & -5.068982 \\ 1 & 2.087754 & -1.152617 & -5.863779 \\ 6 & 0.460947 & 3.117958 & -0.163391 \\ 6 & 0.652494 & 3.987907 & 0.901094 \\ 6 & -0.305046 & 3.552823 & -1.242598 \\ 6 & 0.093395 & 5.257215 & 0.893782 \\ 1 & 1.237703 & 3.674031 & 1.746728 \\ 6 & -0.862268 & 4.818472 & -1.254030 \\ 1 & -0.465747 & 2.893885 & -2.078118 \\ 6 & -0.665274 & 5.677983 & -0.183360 \\ 1 & 0.252837 & 5.913326 & 1.731122 \\ 1 & -1.448926 & 5.134992 & -2.098348 \\ 1 & -1.098471 & 6.662351 & -0.191303 \\ 6 & -0.896149 & 0.933490 & 2.627440 \\ 6 & -0.244604 & 1.556462 & 3.684422 \\ 6 & -2.239404 & 1.231902 & 2.411884 \\ 6 & -0.909836 & 2.457182 & 4.502828 \\ 1 & 0.790920 & 1.333512 & 3.876411 \\ 6 & -2.905194 & 2.130348 & 3.226069 \\ 1 & -2.764587 & 0.762404 & 1.599060 \\ 6 & -2.242772 & 2.748659 & 4.275847 \\ 1 & -0.385125 & 2.926353 & 5.316407 \\ 1 & -3.941841 & 2.349025 & 3.040719 \\ 1 & -2.761467 & 3.447039 & 4.908011 \\ \mathrm{E}(\mathrm{RHF})=-1148.19715139 & \\ \mathrm{ZPE}=240.97111 & & \\ \mathrm{PCM} \mathrm{E}(\mathrm{in} \mathrm{MeNO} 2)=-1148.196532\end{array}$

Tribenzylphosphonium Cation 


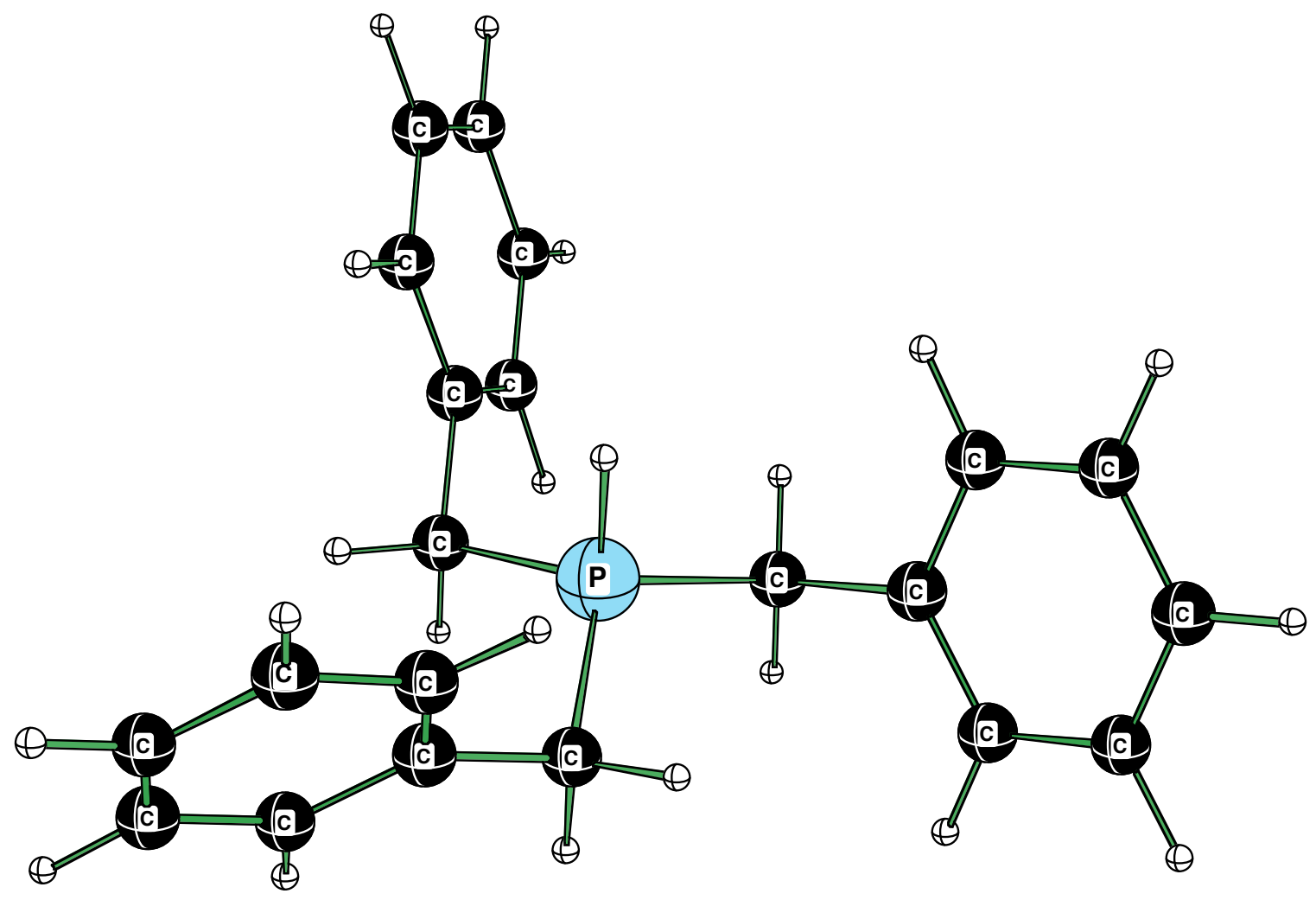

Coordinates

$\begin{array}{cccc}15 & 0.358129 & 1.221213 & 0.197016 \\ 6 & 0.049813 & 1.509487 & 1.974652 \\ 1 & 0.103847 & 2.580150 & 2.135571 \\ 6 & 0.398312 & -0.562200 & -0.197885 \\ 1 & 1.242478 & -0.992707 & 0.331228 \\ 6 & 1.874277 & 2.069516 & -0.368564 \\ 1 & 2.706628 & 1.652911 & 0.189597 \\ 1 & 0.871972 & 1.061212 & 2.523315 \\ 1 & -0.499846 & -0.999712 & 0.222896 \\ 1 & 2.018933 & 1.799052 & -1.408310 \\ 6 & 0.494791 & -0.812217 & -1.690667 \\ 6 & 1.721253 & -1.087713 & -2.283527 \\ 6 & -0.648534 & -0.769511 & -2.481449 \\ 6 & 1.804173 & -1.312611 & -3.648271 \\ 1 & 2.612127 & -1.146472 & -1.682046 \\ 6 & -0.564631 & -0.993259 & -3.845299 \\ 1 & -1.609632 & -0.581133 & -2.033577 \\ 6 & 0.662300 & -1.263097 & -4.431032 \\ 1 & 2.755910 & -1.533539 & -4.095316 \\ 1 & -1.455032 & -0.966426 & -4.446061 \\ 1 & 0.726219 & -1.442277 & -5.488389 \\ 6 & 1.785206 & 3.572992 & -0.189965 \\ 6 & 2.362826 & 4.189310 & 0.913716 \\ 6 & 1.123533 & 4.350649 & -1.134310\end{array}$




$\begin{array}{cccc}6 & 2.276066 & 5.562970 & 1.073436 \\ 1 & 2.897743 & 3.604236 & 1.642029 \\ 6 & 1.036348 & 5.723128 & -0.973381 \\ 1 & 0.691535 & 3.891032 & -2.007188 \\ 6 & 1.610763 & 6.331067 & 0.131927 \\ 1 & 2.733728 & 6.030541 & 1.925718 \\ 1 & 0.530328 & 6.315884 & -1.713001 \\ 1 & 1.547841 & 7.396637 & 0.253566 \\ 6 & -1.285590 & 0.944577 & 2.419363 \\ 6 & -2.455193 & 1.666421 & 2.206451 \\ 6 & -1.356174 & -0.294998 & 3.043900 \\ 6 & -3.677018 & 1.152856 & 2.607157 \\ 1 & -2.414654 & 2.638922 & 1.745592 \\ 6 & -2.579185 & -0.807715 & 3.445592 \\ 1 & -0.457806 & -0.856120 & 3.236161 \\ 6 & -3.740972 & -0.086027 & 3.225498 \\ 1 & -4.573811 & 1.722199 & 2.445727 \\ 1 & -2.621784 & -1.763009 & 3.935552 \\ 1 & -4.688527 & -0.481676 & 3.541406 \\ 1 & -0.702001 & 1.770773 & -0.497130 \\ \mathrm{E}(\mathrm{RHF})=-1148.59594449 & \\ \text { ZPE=248.58409 } & \\ \text { PCM E(in MeNO2)=-1148.656065 }\end{array}$

Methylphosphacyclohexane

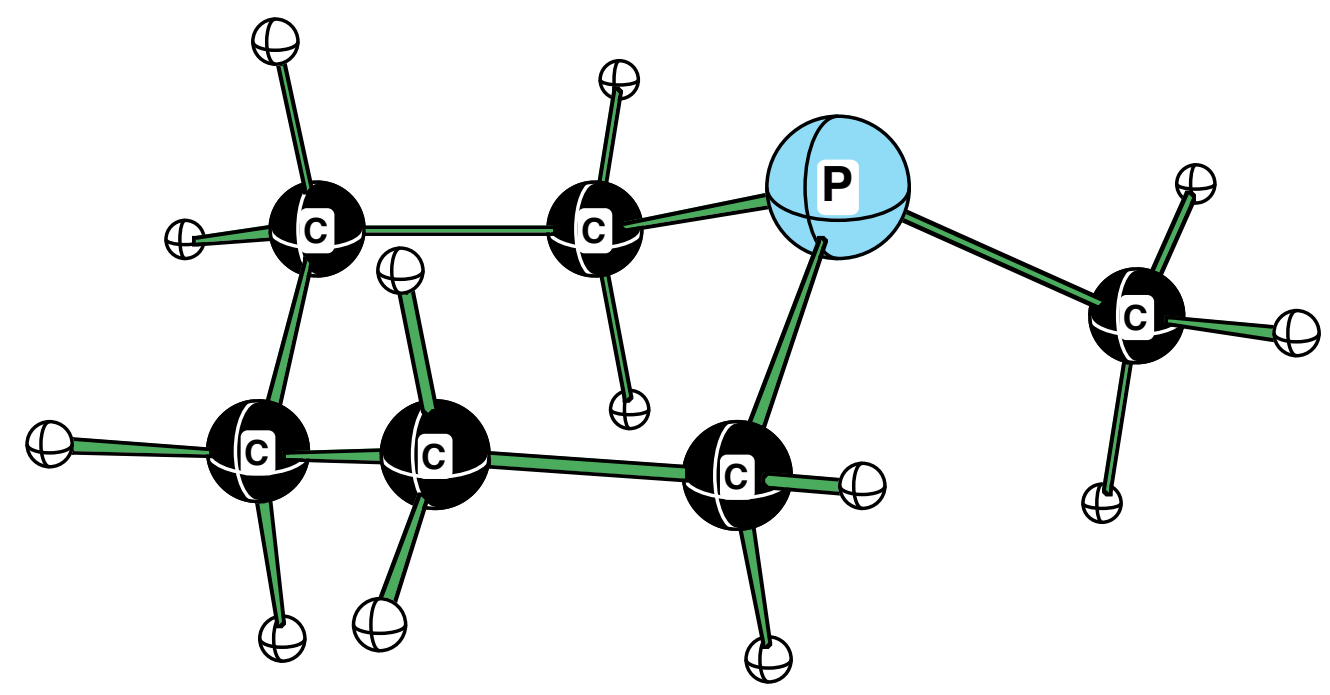

Coordinates
$\begin{array}{llll}6 & -1.235656 & -0.288343 & -1.446973\end{array}$
$\begin{array}{llll}6 & -1.315245 & -0.494976 & 0.073168\end{array}$
$\begin{array}{llll}15 & 0.373661 & -0.803225 & 0.787041\end{array}$
$\begin{array}{llll}6 & 1.172350 & 0.759036 & 0.172628\end{array}$
$\begin{array}{lllll}6 & 1.055957 & 0.866654 & -1.355360\end{array}$ 


$\begin{array}{cccc}6 & -0.397164 & 0.933732 & -1.840437 \\ 1 & -1.767813 & 0.379585 & 0.537232 \\ 1 & -1.953757 & -1.343612 & 0.300753 \\ 1 & -0.817784 & -1.178142 & -1.912936 \\ 1 & -2.240415 & -0.175468 & -1.845498 \\ 1 & 0.708895 & 1.628154 & 0.636181 \\ 1 & 2.217709 & 0.759338 & 0.467407 \\ 1 & 1.586451 & 1.753133 & -1.692745 \\ 1 & 1.552143 & 0.016223 & -1.818020 \\ 1 & -0.865454 & 1.831002 & -1.438757 \\ 1 & -0.407377 & 1.039787 & -2.921970 \\ 6 & 0.094673 & -0.392035 & 2.571186 \\ 1 & -0.394812 & 0.567585 & 2.709252 \\ 1 & -0.517061 & -1.160666 & 3.032784 \\ 1 & 1.044875 & -0.372950 & 3.095430 \\ \mathrm{E}(\mathrm{RHF})=-575.506957334 \\ \text { ZPE=121.13585 }\end{array}$

Protonated Methylphosphacyclohexane Cation

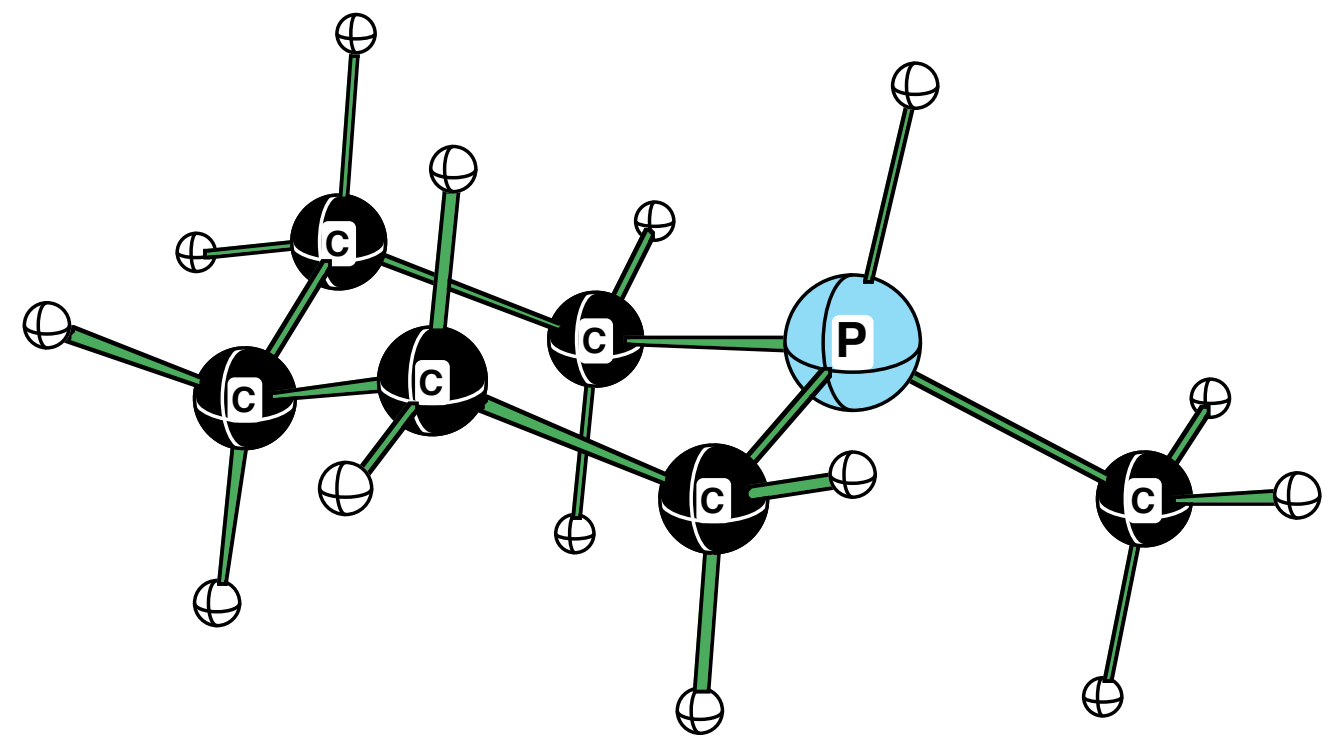

Coordinates

$\begin{array}{cccc}6 & -1.210720 & -0.684337 & -1.374193 \\ 6 & -1.302738 & -0.651450 & 0.166561 \\ 15 & 0.340025 & -0.393492 & 0.892587 \\ 6 & 1.107233 & 0.960005 & -0.041091 \\ 6 & 0.920417 & 0.740671 & -1.557820 \\ 6 & -0.545446 & 0.561480 & -1.973059 \\ 1 & -1.932564 & 0.170720 & 0.494491 \\ 1 & -1.731412 & -1.565847 & 0.561575 \\ 1 & -0.675247 & -1.576410 & -1.687409 \\ 1 & -2.218973 & -0.782208 & -1.757452\end{array}$




$\begin{array}{lrrr}1 & 0.626362 & 1.881775 & 0.274004 \\ 1 & 2.155504 & 1.033184 & 0.226661 \\ 1 & 1.343104 & 1.599610 & -2.064375 \\ 1 & 1.502685 & -0.120113 & -1.875066 \\ 1 & -1.114337 & 1.447374 & -1.700646 \\ 1 & -0.590391 & 0.489510 & -3.053191 \\ 6 & 0.295991 & -0.097598 & 2.677900 \\ 1 & -0.282061 & 0.794158 & 2.889321 \\ 1 & -0.160245 & -0.940888 & 3.182639 \\ 1 & 1.302218 & 0.036977 & 3.056645 \\ 1 & 1.086563 & -1.538086 & 0.674219 \\ \mathrm{E}(\mathrm{RHF})=-575.891738785 & \\ \mathrm{ZPE}=128.49915 & \end{array}$

Bis(2-phenylethyl)phosphine

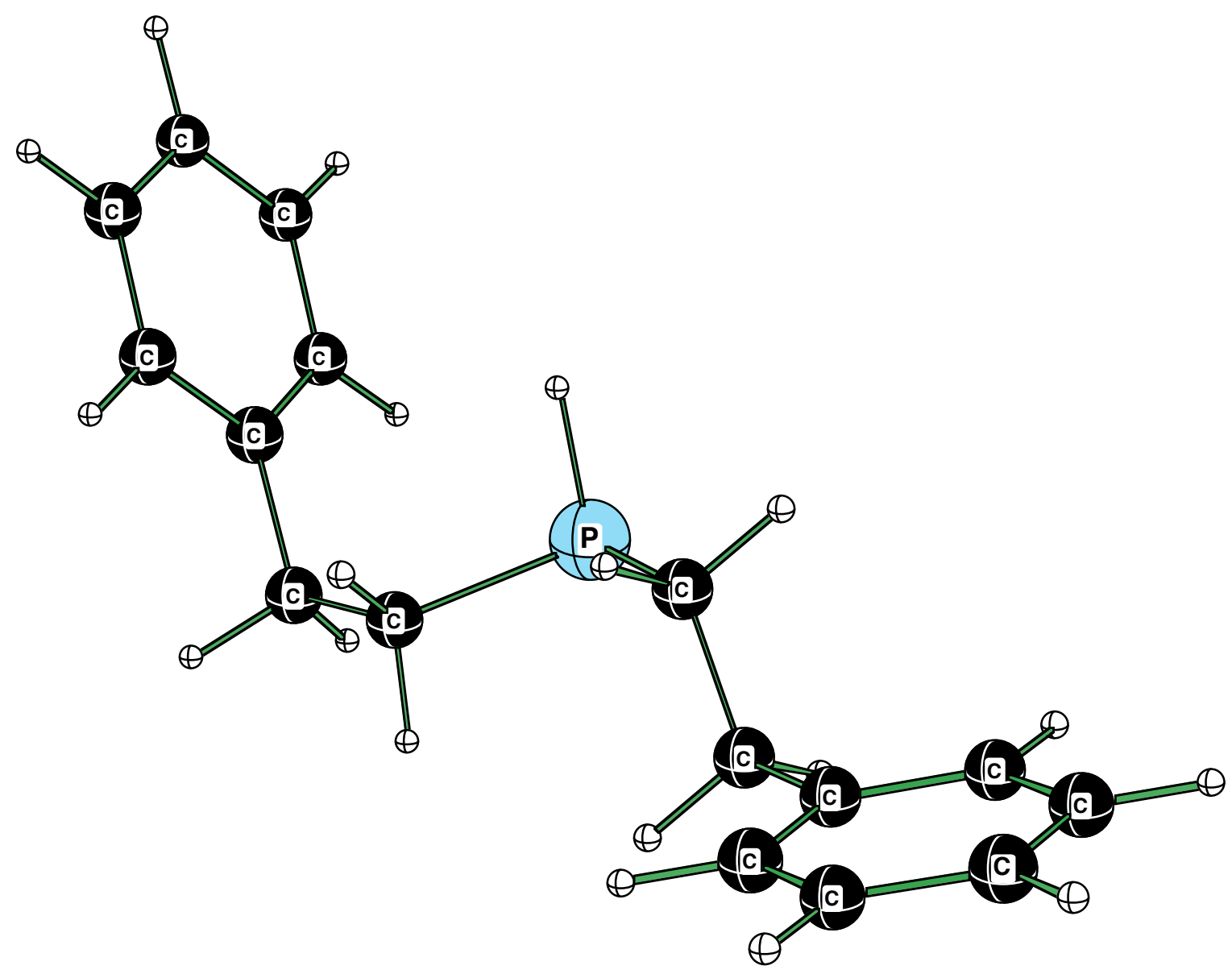

Coordinates

$$
\begin{array}{cccc}
15 & 0.019310 & 0.645636 & -0.080770 \\
6 & -0.256295 & 0.321623 & 1.735152 \\
1 & 0.561363 & -0.257925 & 2.153305 \\
1 & -1.146689 & -0.295947 & 1.812097 \\
1 & 0.105487 & -0.692934 & -0.489030
\end{array}
$$




$\begin{array}{cccc}6 & 1.837576 & 1.065793 & -0.100898 \\ 1 & 1.959758 & 1.958800 & 0.506473 \\ 1 & 2.413921 & 0.279197 & 0.376588 \\ 6 & -0.464319 & 1.605342 & 2.557110 \\ 1 & 0.415802 & 2.237190 & 2.482667 \\ 1 & -1.287015 & 2.171573 & 2.131819 \\ 6 & 2.400054 & 1.359744 & -1.504022 \\ 1 & 1.826180 & 2.161529 & -1.957047 \\ 1 & 3.414784 & 1.731926 & -1.390915 \\ 6 & -0.746226 & 1.317179 & 4.017148 \\ 6 & -2.047994 & 1.121725 & 4.465205 \\ 6 & -2.307559 & 0.827715 & 5.793819 \\ 6 & -1.265425 & 0.725184 & 6.700859 \\ 6 & 0.035831 & 0.920391 & 6.267837 \\ 6 & 0.290275 & 1.214333 & 4.938094 \\ 1 & -2.866399 & 1.204215 & 3.770375 \\ 1 & -3.322243 & 0.683064 & 6.120380 \\ 1 & -1.465306 & 0.499821 & 7.733201 \\ 1 & 0.852191 & 0.847906 & 6.964622 \\ 1 & 1.305567 & 1.369037 & 4.614296 \\ 6 & 2.415024 & 0.162640 & -2.433699 \\ 6 & 3.358425 & -0.848272 & -2.268885 \\ 6 & 3.374271 & -1.950960 & -3.104257 \\ 6 & 2.444056 & -2.063693 & -4.126964 \\ 6 & 1.504207 & -1.064208 & -4.304622 \\ 6 & 1.492582 & 0.040252 & -3.464947 \\ 1 & 4.090707 & -0.768503 & -1.483284 \\ 1 & 4.113207 & -2.719549 & -2.961305 \\ 1 & 2.456235 & -2.919310 & -4.778316 \\ 1 & 0.780315 & -1.138969 & -5.096687 \\ 1 & 0.758332 & 0.812459 & -3.615208 \\ \mathrm{E}(\mathrm{RHF})=-957.681899314 & \\ \mathrm{ZPE}=205.25771 & & \\ & & & \\ 6 & & \\ 6 & & \end{array}$

Bis(2-phenylethyl)phosphonium Cation 


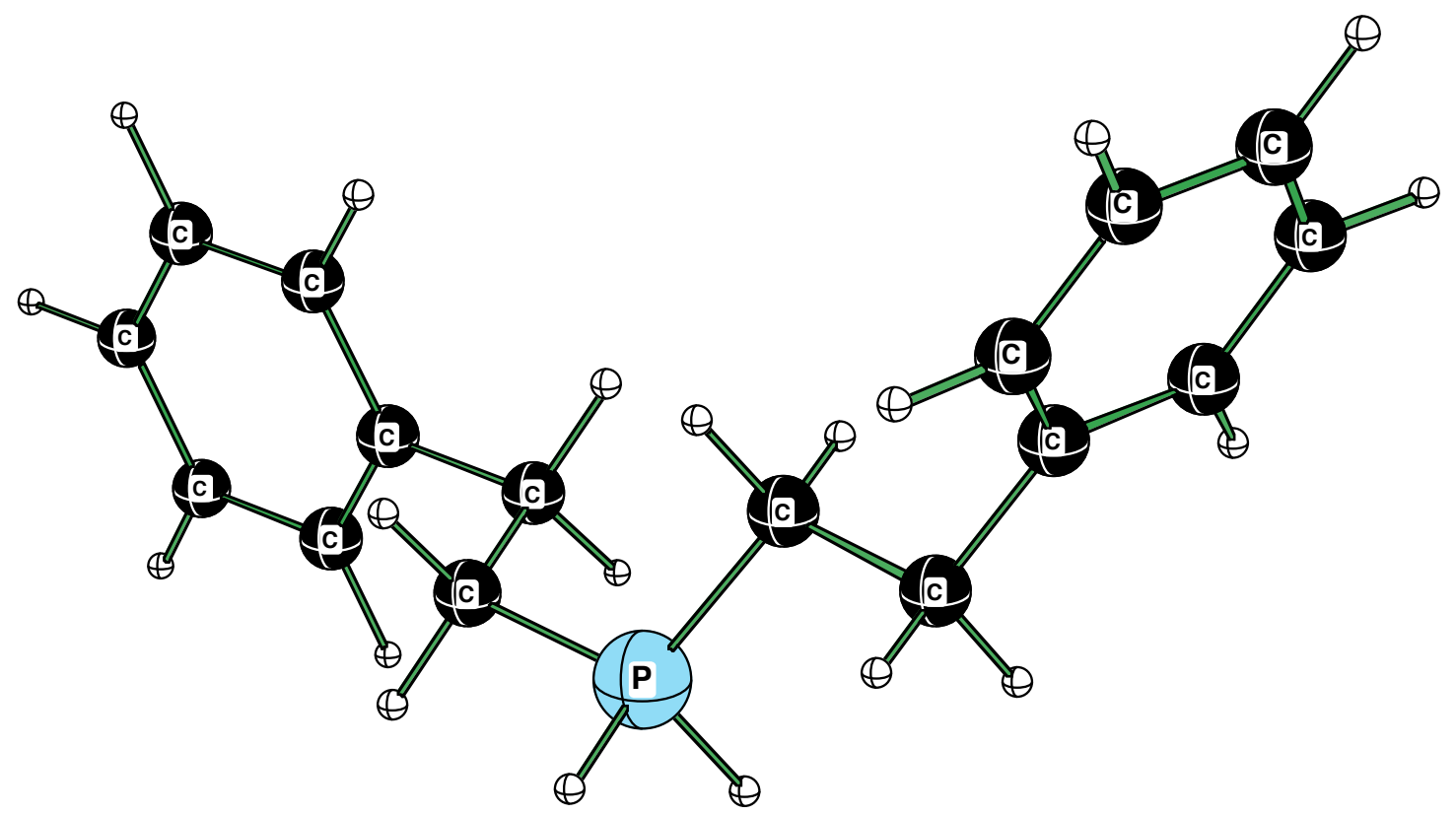

Coordinates

$\begin{array}{cccc}15 & -0.706803 & 0.527031 & -0.024892 \\ 6 & -0.475325 & 0.148786 & 1.742613 \\ 1 & 0.551105 & -0.178479 & 1.871793 \\ 1 & -1.111941 & -0.699766 & 1.970185 \\ 1 & -0.518703 & -0.625307 & -0.766130 \\ 6 & 0.375364 & 1.834071 & -0.684011 \\ 1 & 0.181798 & 2.737898 & -0.116888 \\ 1 & 1.399319 & 1.539115 & -0.478664 \\ 6 & -0.795488 & 1.335323 & 2.679436 \\ 1 & -0.142415 & 2.170723 & 2.451410 \\ 1 & -1.814896 & 1.668225 & 2.511043 \\ 6 & 0.170746 & 2.085500 & -2.195343 \\ 1 & -0.861299 & 2.364329 & -2.382042 \\ 1 & 0.360435 & 1.170092 & -2.746304 \\ 1 & -2.026261 & 0.878076 & -0.246134 \\ 6 & -0.619963 & 0.929702 & 4.127911 \\ 6 & -1.679297 & 0.377763 & 4.837404 \\ 6 & -1.511123 & -0.022379 & 6.152606 \\ 6 & -0.280354 & 0.126704 & 6.771409 \\ 6 & 0.779763 & 0.680072 & 6.071775 \\ 6 & 0.609687 & 1.079791 & 4.756548 \\ 1 & -2.643598 & 0.269166 & 4.370224 \\ 1 & -2.339417 & -0.442110 & 6.693554 \\ 1 & -0.150423 & -0.179493 & 7.793124 \\ 1 & 1.733911 & 0.806753 & 6.549735 \\ 1 & 1.436940 & 1.520407 & 4.226026 \\ 6 & 1.095976 & 3.180806 & -2.682525 \\ 6 & 0.693456 & 4.510012 & -2.646197 \\ 6 & 1.550475 & 5.515258 & -3.062549\end{array}$




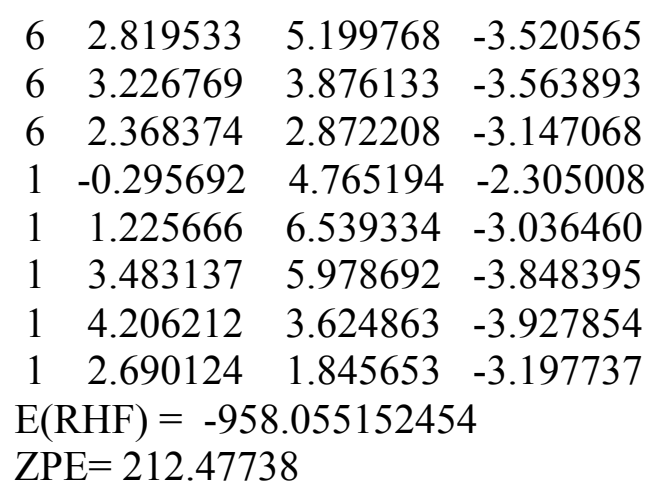

Tris(2-phenylethyl)phosphine

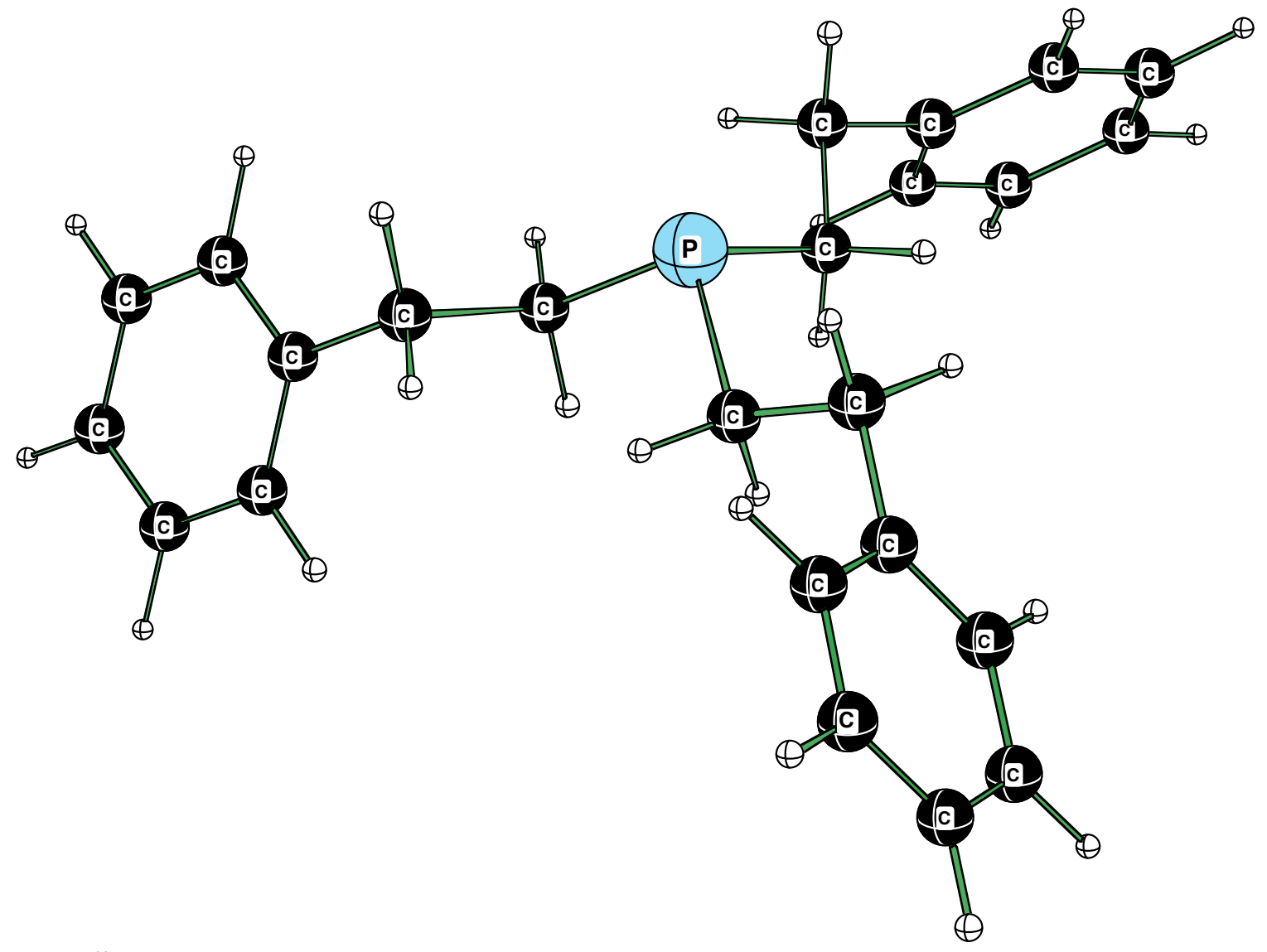

Coordinates

$\begin{array}{cccc}15 & -0.000102 & -0.000032 & -1.271291 \\ 6 & -1.584212 & -0.464513 & -0.405934 \\ 1 & -1.494587 & -0.296004 & 0.664588 \\ 1 & -1.727762 & -1.532685 & -0.540658 \\ 6 & 1.194339 & -1.139639 & -0.406076 \\ 1 & 2.191163 & -0.729888 & -0.540972 \\ 1 & 1.003763 & -1.146241 & 0.664478 \\ 6 & 0.389747 & 1.604219 & -0.406195 \\ 1 & -0.463571 & 2.262583 & -0.540919\end{array}$




$\begin{array}{cccc}1 & 0.490989 & 1.442508 & 0.664342 \\ 6 & 1.177690 & -2.578499 & -0.950933 \\ 1 & 0.188198 & -3.008291 & -0.829681 \\ 1 & 1.376176 & -2.557673 & -2.017750 \\ 6 & -2.821994 & 0.269247 & -0.950867 \\ 1 & -2.699470 & 1.341086 & -0.829754 \\ 1 & -2.903190 & 0.086815 & -2.017664 \\ 6 & 1.644023 & 2.309249 & -0.951344 \\ 1 & 1.526387 & 2.470939 & -2.018090 \\ 1 & 2.510991 & 1.667156 & -0.830507 \\ 6 & 2.192445 & -3.467487 & -0.261982 \\ 6 & 1.859845 & -4.170222 & 0.890848 \\ 6 & 2.795714 & -4.959278 & 1.539443 \\ 6 & 4.085110 & -5.060522 & 1.042986 \\ 6 & 4.428456 & -4.367838 & -0.106492 \\ 6 & 3.488994 & -3.579948 & -0.751538 \\ 1 & 0.859290 & -4.103436 & 1.283276 \\ 1 & 2.516873 & -5.496954 & 2.428468 \\ 1 & 4.812116 & -5.675009 & 1.543360 \\ 1 & 5.425258 & -4.443652 & -0.503799 \\ 1 & 3.765665 & -3.050868 & -1.647648 \\ 6 & 1.906799 & 3.632393 & -0.262206 \\ 6 & 1.355430 & 4.811526 & -0.751064 \\ 6 & 1.568294 & 6.018919 & -0.105823 \\ 6 & 2.340617 & 6.067699 & 1.043148 \\ 6 & 2.898123 & 4.900363 & 1.538908 \\ 6 & 2.682441 & 3.695498 & 0.890129 \\ 1 & 0.758275 & 4.786753 & -1.646767 \\ 1 & 1.135148 & 6.920131 & -0.502580 \\ 1 & 2.509481 & 7.004429 & 1.543674 \\ 1 & 3.503771 & 4.927551 & 2.427540 \\ 1 & 3.125248 & 2.795546 & 1.282019 \\ 6 & -4.099303 & -0.164937 & -0.261908 \\ 6 & -4.845066 & -1.231504 & -0.751431 \\ 6 & -5.997219 & -1.650975 & -0.106431 \\ 6 & -6.425433 & -1.007152 & 1.042972 \\ 6 & -5.692968 & 0.058827 & 1.539394 \\ 6 & -4.541599 & 0.474606 & 0.890842 \\ 1 & -4.525210 & -1.735763 & -1.647477 \\ 1 & -6.561340 & -2.476293 & -0.503712 \\ 1 & -7.321164 & -1.329379 & 1.543314 \\ 1 & -6.019186 & 0.569254 & 2.428360 \\ 1 & -3.983424 & 1.307692 & 1.283244 \\ \mathrm{E}(\mathrm{RHF})=-1265.30137032 & \\ \mathrm{ZPE}=298.61305 & & \\ \mathrm{PCM} & \mathrm{E}(\mathrm{in} \mathrm{MeNO} 2)=-1265.301464\end{array}$

Phosnhines SI v1.1 


\section{Tris(2-phenylethyl)phosphonium Cation}

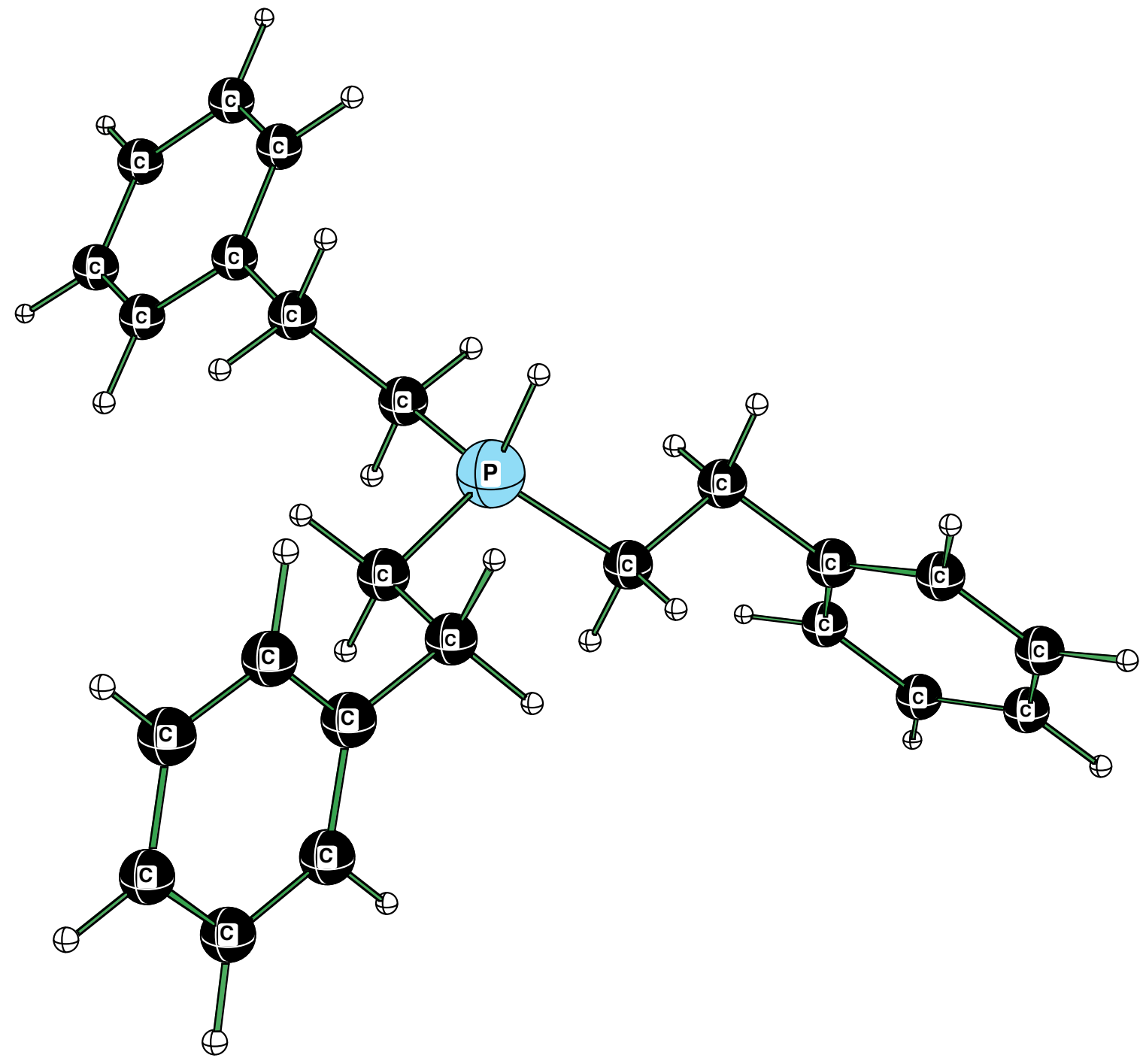

Coordinates

$\begin{array}{cccc}15 & 0.000016 & -0.000004 & -0.659931 \\ 6 & 1.738617 & -0.086490 & -0.118785 \\ 1 & 1.742738 & -0.061239 & 0.966690 \\ 1 & 2.226595 & 0.820929 & -0.456566 \\ 6 & -0.794382 & 1.548912 & -0.118782 \\ 1 & -1.824227 & 1.517798 & -0.456538 \\ 1 & -0.818287 & 1.539866 & 0.966694 \\ 6 & -0.944180 & -1.462437 & -0.118794 \\ 1 & -0.402334 & -2.338741 & -0.456613 \\ 1 & -0.924342 & -1.478663 & 0.966681 \\ 6 & -0.110807 & 2.834571 & -0.634201 \\ 1 & 0.905336 & 2.888187 & -0.260259 \\ 1 & -0.052365 & 2.807202 & -1.717954\end{array}$




$\begin{array}{cccc}6 & 2.510235 & -1.321329 & -0.634178 \\ 1 & 2.048592 & -2.228133 & -0.260216 \\ 1 & 2.457311 & -1.358278 & -1.717929 \\ 6 & -2.399407 & -1.513236 & -0.634144 \\ 1 & -2.404982 & -1.448912 & -1.717894 \\ 1 & -2.953878 & -0.660038 & -0.260152 \\ 1 & 0.000015 & -0.000002 & -2.045697 \\ 6 & -0.879565 & 4.063173 & -0.194131 \\ 6 & -0.585444 & 4.682999 & 1.013850 \\ 6 & -1.308221 & 5.788147 & 1.432261 \\ 6 & -2.333490 & 6.285906 & 0.644586 \\ 6 & -2.630508 & 5.676318 & -0.563705 \\ 6 & -1.906556 & 4.571313 & -0.979951 \\ 1 & 0.217619 & 4.312145 & 1.628243 \\ 1 & -1.066717 & 6.262406 & 2.365995 \\ 1 & -2.891565 & 7.146057 & 0.966099 \\ 1 & -3.418178 & 6.063744 & -1.183844 \\ 1 & -2.137530 & 4.113241 & -1.926953 \\ 6 & 3.958618 & -1.269864 & -0.194116 \\ 6 & 4.912196 & -0.634611 & -0.979972 \\ 6 & 6.231134 & -0.560162 & -0.563720 \\ 6 & 6.610522 & -1.122113 & 0.644608 \\ 6 & 5.666793 & -1.761068 & 1.432319 \\ 6 & 4.348321 & -1.834428 & 1.013904 \\ 1 & 4.631001 & -0.205598 & -1.927002 \\ 1 & 6.960506 & -0.071791 & -1.183886 \\ 1 & 7.634472 & -1.068887 & 0.966124 \\ 1 & 5.956744 & -2.207296 & 2.366082 \\ 1 & 3.625601 & -2.344417 & 1.628322 \\ 6 & -3.079028 & -2.793304 & -0.194087 \\ 6 & -3.005580 & -3.936771 & -0.979912 \\ 6 & -3.600614 & -5.116224 & -0.563704 \\ 6 & -4.277101 & -5.163787 & 0.644554 \\ 6 & -4.358670 & -4.027005 & 1.432238 \\ 6 & -3.762927 & -2.848498 & 1.013866 \\ 1 & -2.493330 & -3.907774 & -1.926880 \\ 1 & -3.542287 & -5.992076 & -1.183846 \\ 1 & -4.743012 & -6.077162 & 0.966037 \\ 1 & -4.890182 & -4.054984 & 2.365949 \\ 1 & -3.843291 & -1.967603 & 1.628265 \\ \mathrm{E}(\mathrm{RHF})=-1265.69287482 & \\ \mathrm{ZPE}=305.96493 & & \\ \text { PCM E(in MeNO2)=-1265.760062 }\end{array}$

(2-Cyanoethyl)dimethyl phosphine 


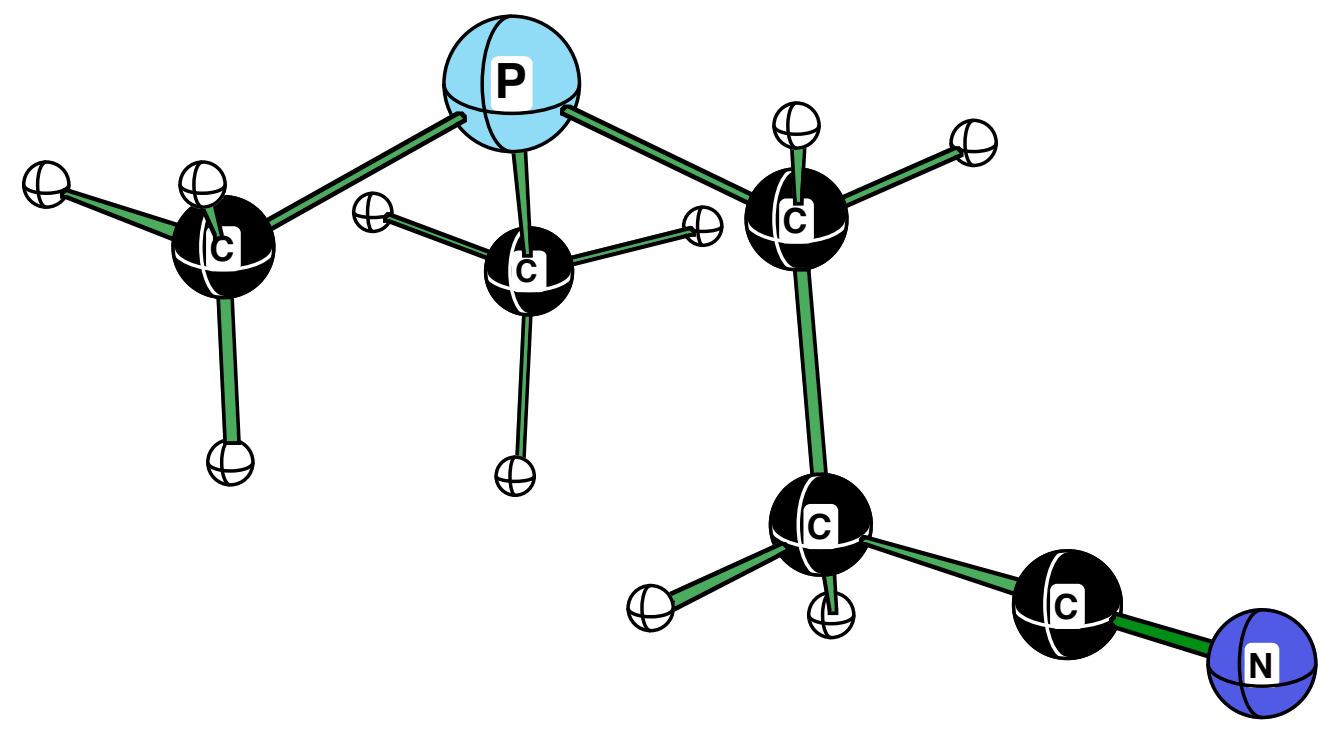

Coordinates

$\begin{array}{lccc}15 & -1.290593 & 0.961614 & 0.125847 \\ 6 & -0.943980 & 0.913274 & 1.945773 \\ 1 & 0.112442 & 0.908332 & 2.194418 \\ 1 & -1.392331 & 1.785963 & 2.408938 \\ 1 & -1.409591 & 0.037552 & 2.386253 \\ 6 & -0.133689 & 2.308468 & -0.405344 \\ 1 & -0.074173 & 2.336933 & -1.488559 \\ 1 & -0.535265 & 3.261695 & -0.077899 \\ 1 & 0.870011 & 2.212747 & -0.003723 \\ 6 & -0.426119 & -0.572583 & -0.486639 \\ 1 & -1.011968 & -1.417971 & -0.139450 \\ 1 & -0.519571 & -0.570144 & -1.568166 \\ 6 & 1.047952 & -0.786449 & -0.105524 \\ 1 & 1.670024 & 0.021125 & -0.470559 \\ 1 & 1.173930 & -0.833070 & 0.968886 \\ 6 & 1.573547 & -2.042665 & -0.669844 \\ 7 & 1.967018 & -3.012520 & -1.109767 \\ \text { E(RHF)=-590.330697139 } \\ \text { ZPE=95.03473 } \\ \text { E(in solution)= -590.332894 }\end{array}$

(2-Cyanoethyl)dimethyl phosphonium Cation 


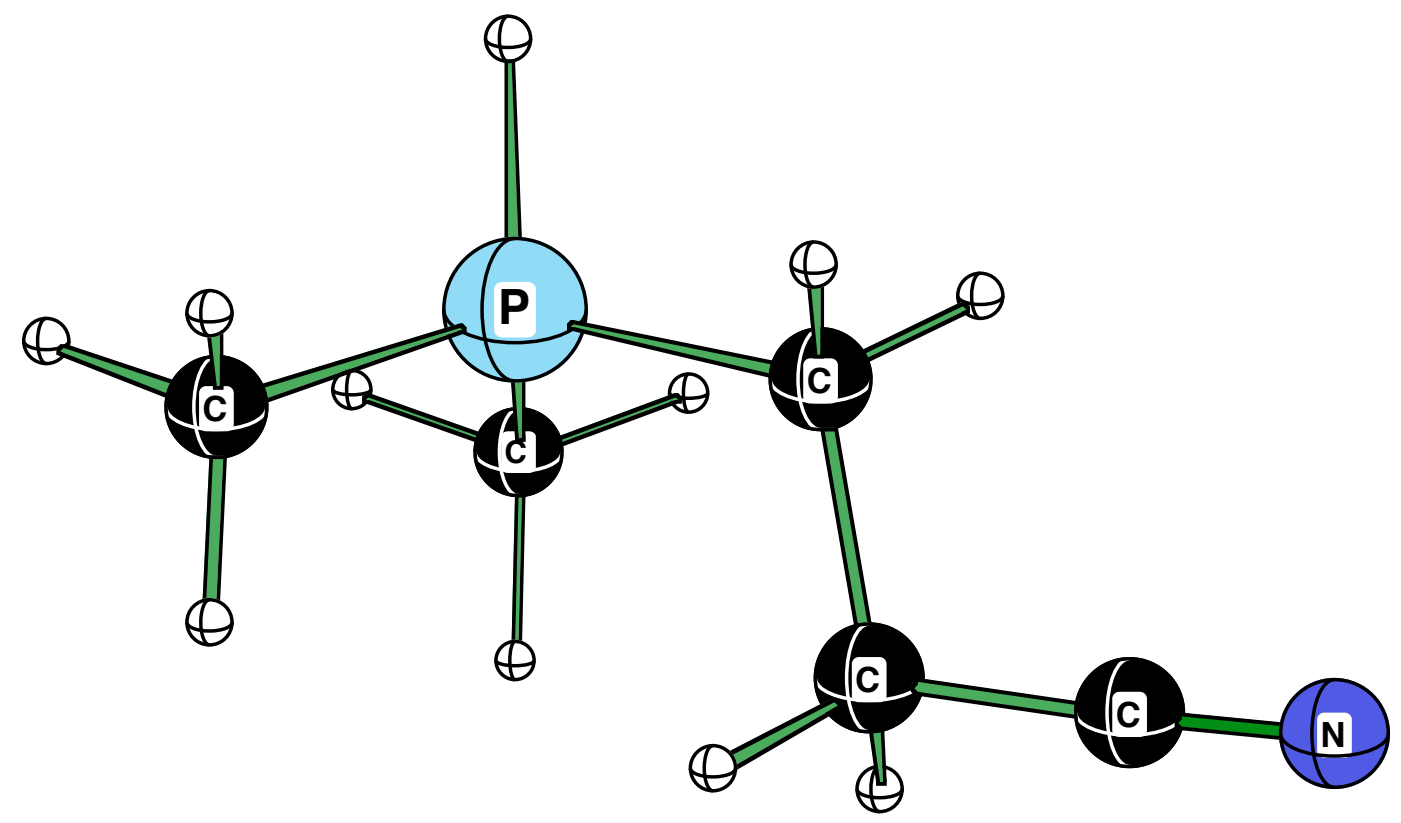

Coordinates

$\begin{array}{rrrr}15 & 0.450987 & 0.789621 & 1.089523 \\ 1 & 0.357735 & 0.629530 & 2.458984 \\ 6 & 2.209632 & 0.855128 & 0.662796 \\ 1 & 2.334801 & 1.024749 & -0.399937 \\ 1 & 2.684484 & 1.666766 & 1.201400 \\ 1 & 2.693656 & -0.075652 & 0.933772 \\ 6 & -0.381717 & 2.339194 & 0.659815 \\ 1 & -1.429929 & 2.285920 & 0.929020 \\ 1 & 0.077093 & 3.160018 & 1.198402 \\ 1 & -0.296911 & 2.531935 & -0.402964 \\ 6 & -0.379135 & -0.661318 & 0.359856 \\ 1 & 0.120026 & -1.542939 & 0.748090 \\ 1 & -1.392809 & -0.676513 & 0.746351 \\ 6 & -0.395402 & -0.692856 & -1.181766 \\ 1 & -0.912630 & 0.166125 & -1.592001 \\ 1 & 0.607921 & -0.704809 & -1.590261 \\ 6 & -1.088644 & -1.904085 & -1.639053 \\ 7 & -1.628092 & -2.846545 & -1.963371\end{array}$

$\mathrm{E}(\mathrm{RHF})=-590.693513358$

$\mathrm{ZPE}=102.24747$

$\mathrm{PCM} E($ in MeNO2 $)=-590.782239$

Tris-(2-cyanoethyl)phosphine 


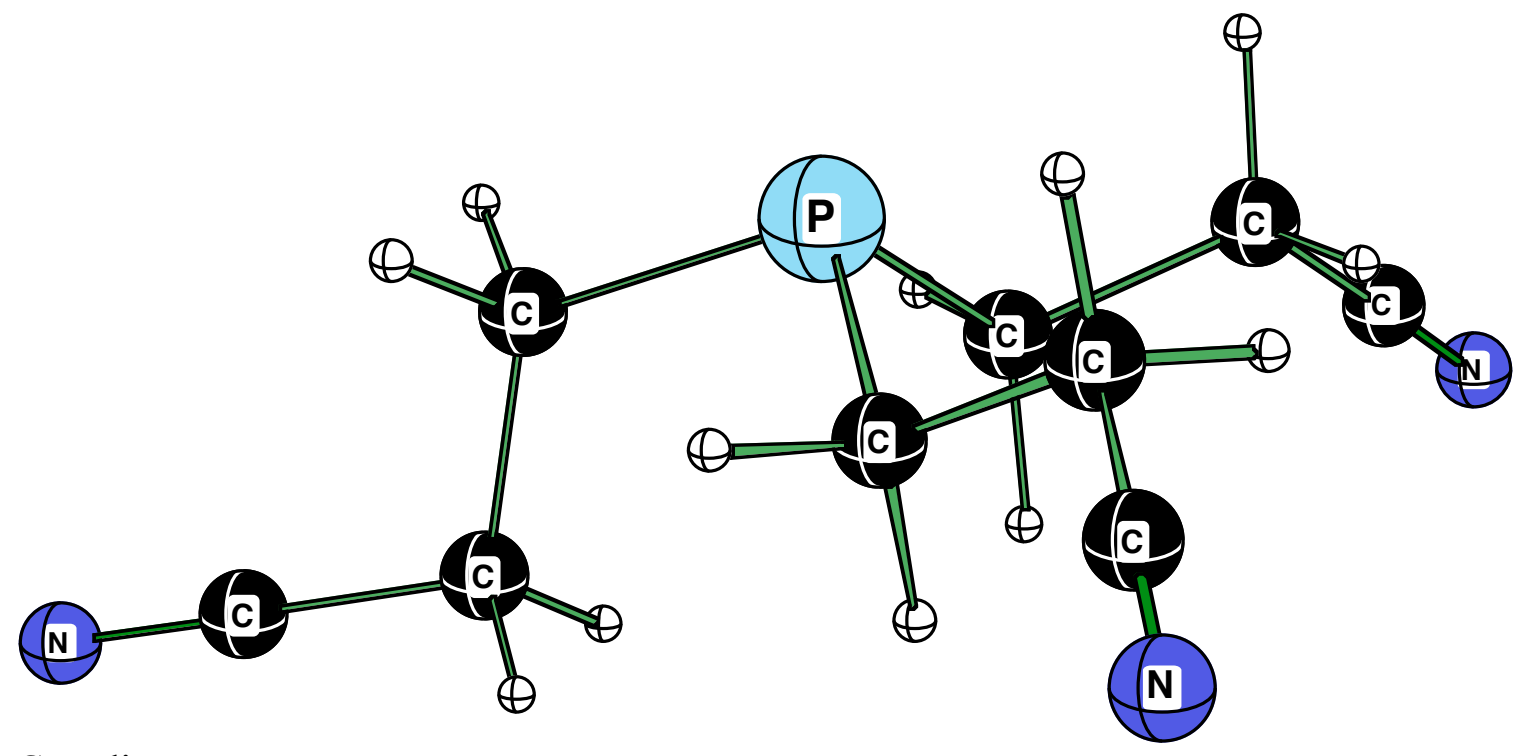

Coordinates

$\begin{array}{cccc}15 & -0.915042 & -0.050112 & -0.640584 \\ 6 & -0.899118 & -0.076493 & 1.226907 \\ 1 & 0.109392 & -0.113319 & 1.624802 \\ 1 & -1.395659 & -0.991812 & 1.533625 \\ 6 & 0.656060 & 0.865141 & -1.067751 \\ 1 & 1.008370 & 0.463078 & -2.012557 \\ 1 & 1.439094 & 0.692467 & -0.337124 \\ 6 & -0.421241 & -1.802193 & -1.024872 \\ 1 & -1.262607 & -2.428839 & -0.747430 \\ 1 & -0.342515 & -1.871763 & -2.104927 \\ 6 & 0.862123 & -2.356220 & -0.382378 \\ 1 & 1.730499 & -1.776424 & -0.669621 \\ 1 & 0.802924 & -2.338155 & 0.698784 \\ 6 & 0.433576 & 2.377443 & -1.238905 \\ 1 & -0.324828 & 2.569189 & -1.988209 \\ 1 & 0.097159 & 2.837839 & -0.317677 \\ 6 & -1.654517 & 1.113143 & 1.843333 \\ 1 & -1.190488 & 2.057400 & 1.584766 \\ 1 & -2.674337 & 1.147381 & 1.479745 \\ 6 & 1.099830 & -3.750762 & -0.793704 \\ 6 & 1.672873 & 3.059259 & -1.649869 \\ 6 & -1.690078 & 1.023070 & 3.313110 \\ 7 & -1.707911 & 0.939306 & 4.444766 \\ 7 & 1.267691 & -4.824505 & -1.120690 \\ 7 & 2.636159 & 3.569531 & -1.965595 \\ \mathrm{E}(\mathrm{RHF})=-851.855195434 & \\ \mathrm{ZPE}=133.26785 & & \\ \mathrm{E}(\mathrm{in} & 501 \mathrm{ition})=-851.874407 \\ \end{array}$




\section{Tris-(2-cyanoethyl)phosphonium Cation}

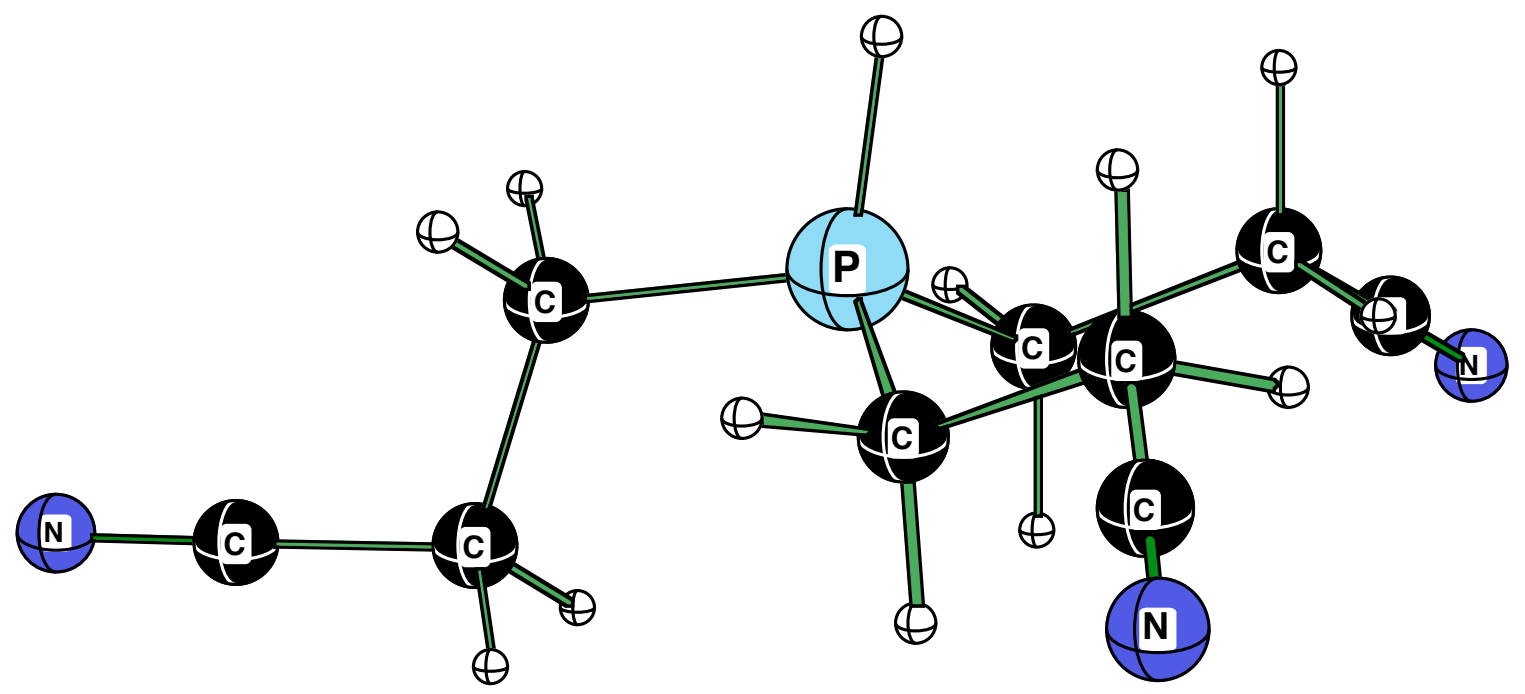

Coordinates

$\begin{array}{cccc}15 & -0.593481 & -0.153409 & -0.463648 \\ 6 & -0.889253 & -0.055485 & 1.338247 \\ 1 & 0.072212 & -0.110781 & 1.837745 \\ 1 & -1.437815 & -0.952710 & 1.605724 \\ 6 & 0.733289 & 0.949720 & -1.069271 \\ 1 & 1.024001 & 0.569812 & -2.043245 \\ 1 & 1.590083 & 0.832100 & -0.414258 \\ 6 & -0.248402 & -1.879043 & -0.951489 \\ 1 & -1.114405 & -2.468075 & -0.668524 \\ 1 & -0.193714 & -1.897664 & -2.034841 \\ 6 & 1.033138 & -2.484638 & -0.340753 \\ 1 & 1.915909 & -1.927874 & -0.631664 \\ 1 & 0.990329 & -2.501475 & 0.741734 \\ 6 & 0.354522 & 2.440829 & -1.192433 \\ 1 & -0.498999 & 2.577650 & -1.845822 \\ 1 & 0.113257 & 2.876878 & -0.231820 \\ 6 & -1.670821 & 1.185900 & 1.817957 \\ 1 & -1.130406 & 2.103165 & 1.623779 \\ 1 & -2.635866 & 1.256437 & 1.330254 \\ 6 & 1.186962 & -3.865984 & -0.814003 \\ 6 & 1.491433 & 3.180642 & -1.754805 \\ 6 & -1.890275 & 1.085555 & 3.266378 \\ 7 & -2.040861 & 0.978049 & 4.384414 \\ 7 & 1.277548 & -4.928728 & -1.196810 \\ 7 & 2.387749 & 3.721604 & -2.188642 \\ 1 & -1.766981 & 0.202216 & -1.106032 \\ \mathrm{E}(\mathrm{RHF})=-852.188433443 & \\ \mathrm{ZPE}=140.25243 & & \\ \mathrm{PCM} \mathrm{E}(\mathrm{in} \mathrm{MeNO})=-852.309307\end{array}$


Tris-(2-methoxyethyl)phosphine

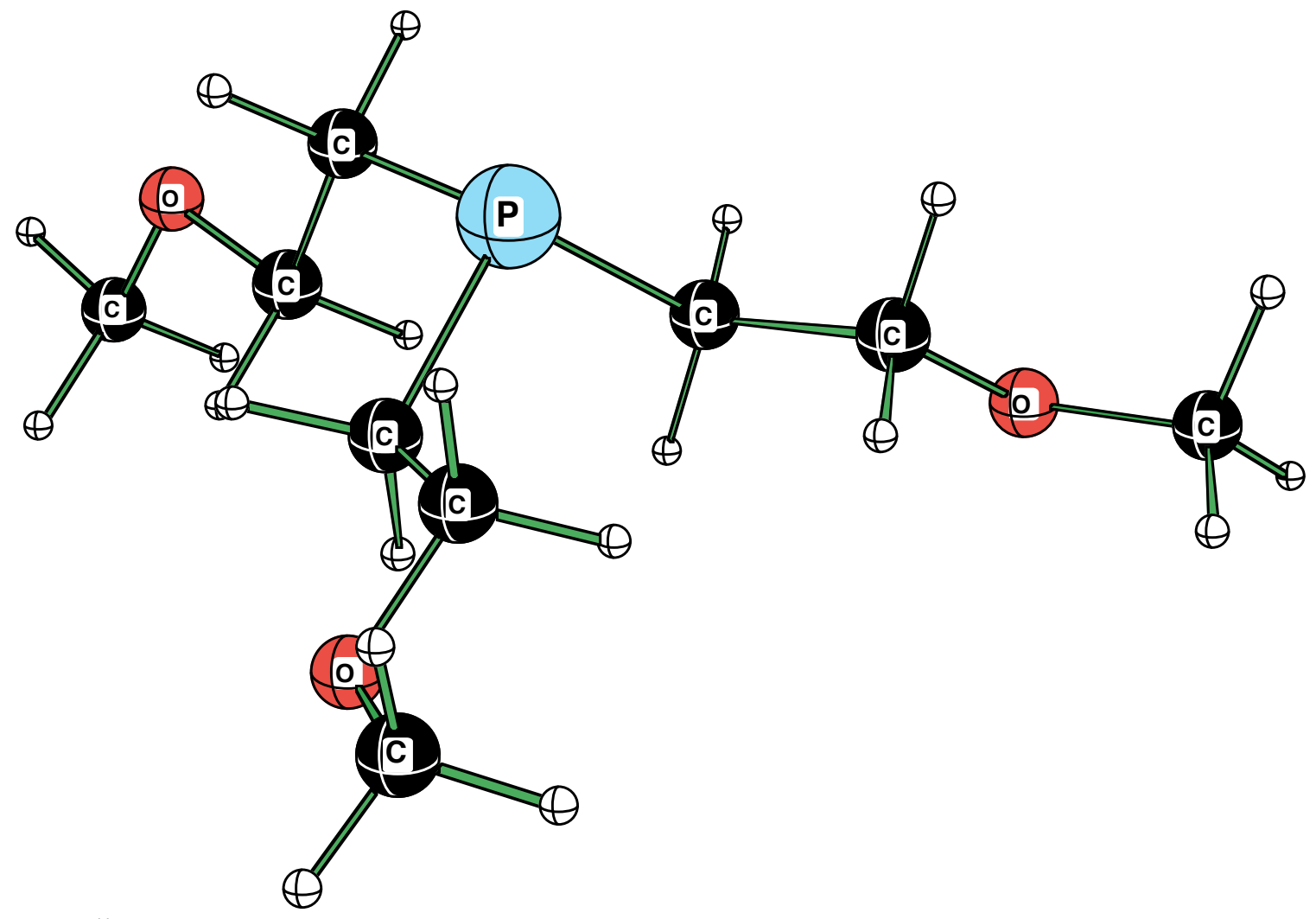

Coordinates

$\begin{array}{cccc}15 & -0.921605 & -0.549628 & -0.480836 \\ 6 & -0.483895 & -0.446895 & 1.330338 \\ 1 & 0.539482 & -0.120668 & 1.484370 \\ 1 & -0.563586 & -1.449399 & 1.740566 \\ 6 & 0.123726 & 0.791886 & -1.249107 \\ 1 & 0.378569 & 0.468822 & -2.254255 \\ 1 & 1.057305 & 0.936927 & -0.715391 \\ 6 & 0.009624 & -2.074774 & -0.993834 \\ 1 & -0.491204 & -2.922078 & -0.535262 \\ 1 & -0.130935 & -2.187665 & -2.064598 \\ 6 & 1.496925 & -2.164321 & -0.686467 \\ 1 & 2.042856 & -1.352245 & -1.162832 \\ 1 & 1.678303 & -2.095739 & 0.384432 \\ 8 & 1.958559 & -3.392385 & -1.167810 \\ 6 & 3.316291 & -3.614324 & -0.954010 \\ 1 & 3.559095 & -3.610146 & 0.107112 \\ 1 & 3.557033 & -4.585524 & -1.364047 \\ 1 & 3.926636 & -2.861667 & -1.449935 \\ 6 & -0.595171 & 2.128165 & -1.360585 \\ 1 & -1.513794 & 2.015538 & -1.933868\end{array}$




$\begin{array}{cccc}1 & -0.872552 & 2.507593 & -0.377603 \\ 6 & -1.415641 & 0.454628 & 2.126562 \\ 1 & -1.374471 & 1.480751 & 1.762503 \\ 1 & -2.445584 & 0.117089 & 2.024162 \\ 8 & 0.262186 & 3.032128 & -1.990897 \\ 6 & -0.280971 & 4.301056 & -2.172873 \\ 1 & 0.465372 & 4.908190 & -2.666453 \\ 1 & -1.174500 & 4.267310 & -2.793446 \\ 1 & -0.539167 & 4.764669 & -1.222312 \\ 8 & -1.022303 & 0.412643 & 3.465602 \\ 6 & -1.808334 & 1.186299 & 4.315263 \\ 1 & -1.777422 & 2.238932 & 4.039259 \\ 1 & -2.846203 & 0.858576 & 4.307217 \\ 1 & -1.414013 & 1.075749 & 5.316039 \\ \mathrm{E}(\mathrm{RHF})=-918.288260385 & \end{array}$

$\mathrm{ZPE}=202.28139$

$\mathrm{PCM} E($ in $\mathrm{MeNO} 2)=-918.281341$

Tris-(2-methoxyethyl)phosphonium Cation

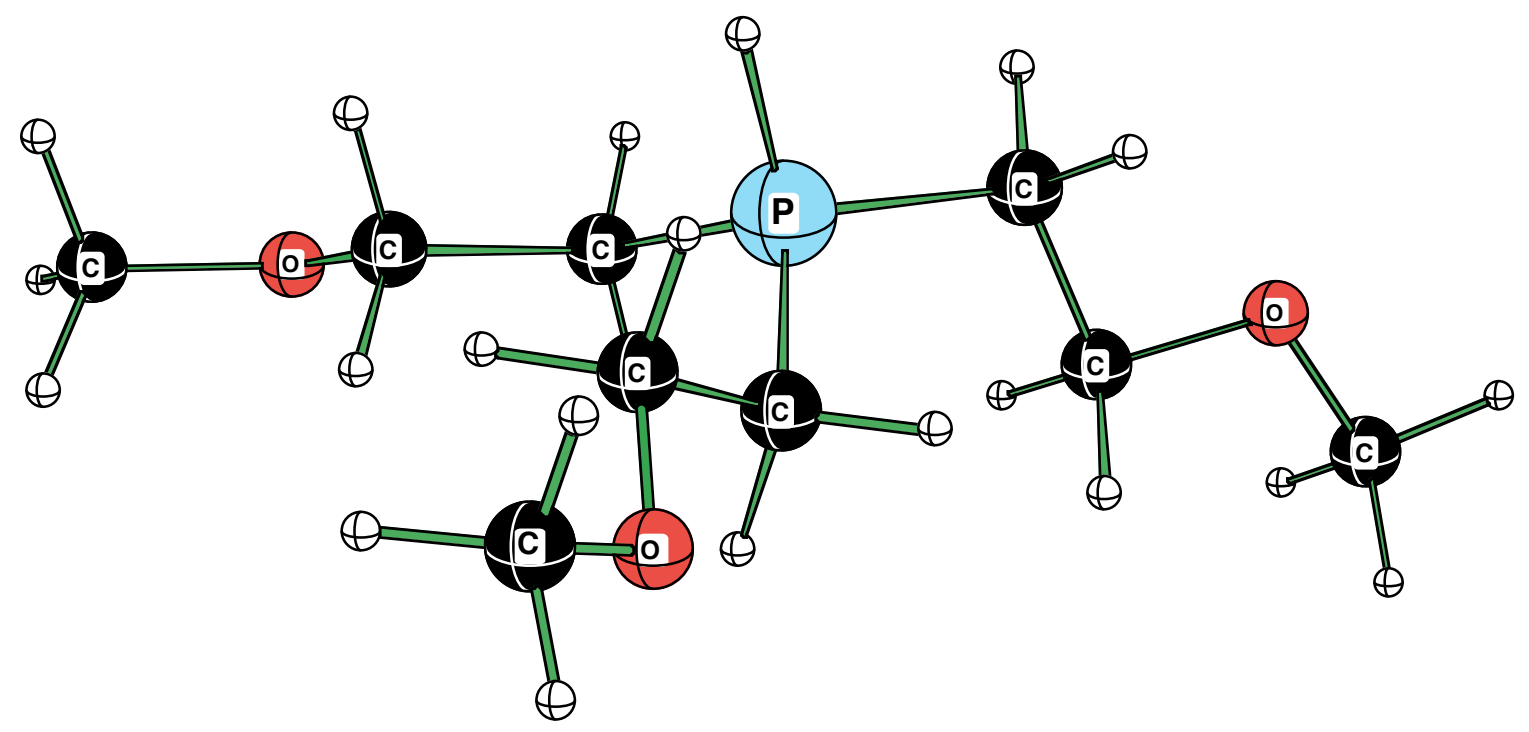

Coordinates

$\begin{array}{cccc}15 & -0.504410 & -0.420344 & -0.565521 \\ 6 & -0.944917 & -0.591710 & 1.190860 \\ 1 & -0.030280 & -0.741038 & 1.754619 \\ 1 & -1.530192 & -1.500524 & 1.285514 \\ 6 & 0.571500 & 1.013001 & -0.903950 \\ 1 & 1.204407 & 0.762165 & -1.747787 \\ 1 & 1.222312 & 1.162558 & -0.049276 \\ 6 & 0.231698 & -1.960159 & -1.194513 \\ 1 & -0.493648 & -2.754921 & -1.057571 \\ 1 & 0.375635 & -1.838096 & -2.262866 \\ 6 & 1.562311 & -2.372181 & -0.554348\end{array}$




$\begin{array}{cccc}1 & 2.308485 & -1.586579 & -0.668610 \\ 1 & 1.437472 & -2.558114 & 0.511122 \\ 8 & 1.944128 & -3.520162 & -1.217736 \\ 6 & 3.152392 & -4.084546 & -0.772357 \\ 1 & 3.086326 & -4.368448 & 0.273646 \\ 1 & 3.326831 & -4.964664 & -1.371049 \\ 1 & 3.977281 & -3.390719 & -0.904127 \\ 1 & -1.679845 & -0.239303 & -1.274481 \\ 6 & -0.164732 & 2.321214 & -1.216998 \\ 1 & -0.769476 & 2.207177 & -2.115644 \\ 1 & -0.833915 & 2.602101 & -0.404690 \\ 6 & -1.732956 & 0.568241 & 1.807637 \\ 1 & -1.143111 & 1.483015 & 1.801900 \\ 1 & -2.646351 & 0.756797 & 1.243847 \\ 8 & 0.824106 & 3.265929 & -1.394616 \\ 6 & 0.360981 & 4.548642 & -1.737148 \\ 1 & 1.230421 & 5.178018 & -1.843893 \\ 1 & -0.184814 & 4.526796 & -2.675490 \\ 1 & -0.280545 & 4.951011 & -0.958736 \\ 8 & -2.016821 & 0.170024 & 3.097937 \\ 6 & -2.741953 & 1.104362 & 3.858021 \\ 1 & -2.189339 & 2.033361 & 3.962159 \\ 1 & -3.706599 & 1.309883 & 3.403751 \\ 1 & -2.892154 & 0.667182 & 4.832587 \\ \mathrm{E}(\mathrm{RHF})=-918.669402202 & \\ \text { ZPE }=209.45114 & \\ \text { PCM E(in MeNO2)=-918. } & \\ & & \end{array}$




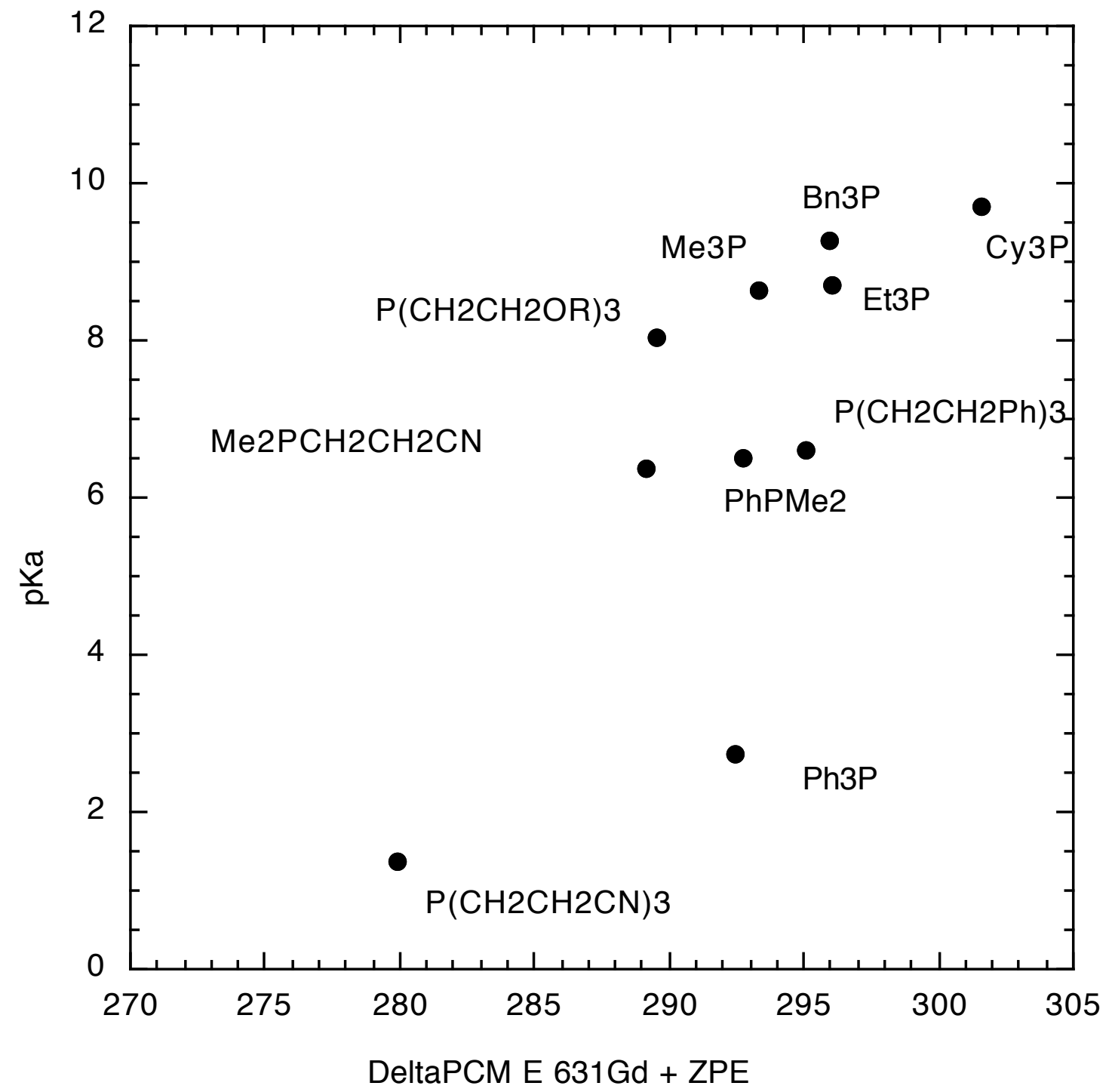

Fig S4. pKa of phosphonium ions in nitromethane compared to the Polarized Continuum Model (PCM), $\Delta \mathrm{E}+\mathrm{ZPE}$.

Table S3. Computed PA of some phosphines (HF E+ZPE, 6-31G*, $\mathrm{Kcal} \mathrm{mol}^{-1}$ )

\begin{tabular}{|c|c|c|}
\hline Phosphine & PA, R=isopropyl & PA, R= benzyl \\
\hline $\mathrm{RPH}_{2}$ & 210.5 & 211.1 \\
$\mathrm{R}_{2} \mathrm{PH}$ & 226.8 & 227.5 \\
$\mathrm{R}_{3} \mathrm{P}$ & 241.9 & 242.6 \\
\hline
\end{tabular}

